\title{
Nothing but coincidences: the point-coincidence and Einstein's struggle with the meaning of coordinates in physics
}

\section{Marco Giovanelli ${ }^{1}$ iD}

Received: 29 August 2019 / Accepted: 16 November 2020 / Published online: 4 May 2021

(C) The Author(s) 2021, corrected publication 2022

\begin{abstract}
In his 1916 review paper on general relativity, Einstein made the often-quoted oracular remark that all physical measurements amount to a determination of coincidences, like the coincidence of a pointer with a mark on a scale. This argument, which was meant to express the requirement of general covariance, immediately gained great resonance. Philosophers such as Schlick found that it expressed the novelty of general relativity, but the mathematician Kretschmann deemed it as trivial and valid in all spacetime theories. With the relevant exception of the physicists of Leiden (Ehrenfest, Lorentz, de Sitter, and Nordström), who were in epistolary contact with Einstein, the motivations behind the point-coincidence remark were not fully understood. Only at the turn of the 1960s did Bergmann (Einstein's former assistant in Princeton) start to use the term 'coincidence' in a way that was much closer to Einstein's intentions. In the 1980s, Stachel, projecting Bergmann's analysis onto his historical work on Einstein's correspondence, was able to show that what he started to call 'the point-coincidence argument' was nothing but Einstein's answer to the infamous 'hole argument'. The latter has enjoyed enormous popularity in the following decades, reshaping the philosophical debate on spacetime theories. The point-coincidence argument did not receive comparable attention. By reconstructing the history of the argument and its reception, this paper argues that this disparity of treatment is not justified. This paper will also show that the notion that only coincidences are observable in physics marks every critical step of Einstein's struggle with the meaning of coordinates in physics.
\end{abstract}

Keywords General relativity · Point-coincidence argument · Hole argument · Observables · Operationalism

Marco Giovanelli

marco.giovanelli@unito.it

1 Department of Philosophy and Educational Sciences, Università degli Studi di Torino, Via S.

Ottavio, Torino, 20 10124, Italy 
I was too much a prisoner of the idea that our equations must fully reproduce [...] the relations between the phenomena and the chosen coordinate system, whereas we can be happy if they duly reproduce the mutual relations between the phenomena

—Lorentz to Ehrenfest, Jan. 11, 1916

\section{Introduction}

In one of the opening sections of his 1916 review paper on general relativity, Einstein (1916a, 776-777) made the often-quoted remark that all our measurements amount to the determination of spacetime coincidences, like the coincidence of a pointer with a mark on a scale. Such statements did not entail any reference to coordinates, lending support to Einstein's stance that there is no reason to privilege one coordinate system over another. The sudden appearance of the 'coincidences'parlance in Einstein's writings gained immediate resonance. Philosophers such as Moritz Schlick (1917) found that Einstein's coincidence remark aptly expressed the conceptual novelty of general relativity with respect to previous spacetime theories. On the contrary, the young mathematician Erich Kretschmann (1917) deemed the argument as trivial and actually applicable in all spacetime theories. Kretschmann's objection was quite serious but was initially neglected; on the contrary, Schlick's interpretation was essentially off-track but enjoyed significant success (Giovanelli 2013). However, in hindsight, despite the fact that both Schlick (Engler and Renn 2013) and Kretschmann (Howard and Norton 1993) have been credited for suggesting this argument to Einstein, both readings could not fully grasp the problem that Einstein's remark was trying to address, a problem that remained hidden in Einstein's private correspondence.

Nevertheless, led astray by Einstein's elliptic formulation, philosophers of the most disparate schools, in a case of intellectual pareidolia, believed to see a confirmation of their views in Einstein's reduction of all measurements for the assessment of coincidences (Cassirer 1921, 83; Petzoldt 1921, 64; see Howard 1991). Not surprisingly, the argument continued to enjoy certain popularity among philosophers for several decades (e.g., Reichenbach 1924, 1928; Popper 1929). On the contrary, although physicists continued to struggle with the physical meaning of coordinates in general relativity (Darrigol 2015), there is no evidence that Einstein appealed to the observability of coincidences again besides a cursory remark in his 1919 Berlin lectures on general relativity. ${ }^{1}$ While he was moving toward the unified field theory project, the positivist undertones of the language of 'coincidences' had probably become a liability rather than an asset (Giovanelli 2016; 2018). As far as I can see, it was not until the turn of the 1960s that Peter G. Bergmann (1961), former assistant of Einstein at Princeton, used the language of 'coincidences' in order to address the problem of observables in general relativity.

\footnotetext{
${ }^{1}$ This is at least the last instance I was able to find.
} 
In the early 1980s, John Stachel (1980), in a famous talk at the University of Jena, applied the gist of Bergmann's 'measurability analysis' (Bergmann and Smith 1982) to his archival work on Einstein's correspondence (Stachel 1993). Stachel realized that what he started to call the 'point-coincidence argument' was nothing but an attempt to overcome the infamous 'hole argument', Einstein's refutation of the predictability of a set of field equations holding in any coordinate system. The argument was first mentioned in print by Roberto Torretti (1983), but a detailed historical reconstruction was laid down by John Norton $(1984,1987)$ in his pioneering investigations on Einstein's path to the 1915 field equations. Starting from a classical paper that Norton wrote soon thereafter with John Earman (1987), the 'hole argument' has started to enjoy enormous popularity in philosophical (see Rickles 2007, for an overview) and, to a lesser extent, physical literature (see Stachel 2014, for an overview). In particular, it was pivotal for the revival of the substantivalism/relationalism debate that dominated the philosophical discussions in the ensuing decades (Pooley 2013). By contrast, the point-coincidence argument seemed to have been relegated to the rank of antiquarian curiosity. The literature on this topic has usually focused on philosophers' misunderstanding of Einstein's remark (Ryckman 1992; Howard et al. 1999) rather than on the actual impact of the point-coincidence argument on the history of physics. Over a century has now passed since the argument was last used by Einstein. Thus, this might be a good opportunity to try and reassess the meaning of Einstein's declaration that only coincidences are observable in physics, also in light of additional textual evidence that has recently emerged.

The goal of the present paper is to provide a reasonably self-contained and historically accurate overview of the history of the 'point-coincidence argument'. For this purpose, the paper attempts (a) to insert some sparse material with which Einstein scholars might already be familiar into a broader and unitary narrative arc. In particular, it will be shown that the appearance of the point-coincidences parlance in Einstein's writings marks all the main stages of Einstein's struggle with the meaning of coordinates in physics: from Einstein's reliance on the idea of a coordinate scaffolding (Section 2.1) to its Aufhebung within the framework of special relativity (Section 2.3); from his recognition of the physical insignificance of coordinates (Section 2.3) to his concerns about its consequences for physics' predictability (Section 3.1); and from his apparent full acceptance of the immateriality of the coordinate systems (Section 5) to the reemergence of never-fading nostalgia for the idea of a physically preferred class of coordinates (Section 6.1).

Moreover, (b) the paper will integrate this material with a recently published correspondence $^{2}$ with (Section 4.1) and within (Section 4.2) the Leiden physics community (Hendrik A. Lorentz, Paul Ehrenfest, Willem de Sitter, Johannes Droste, and Gunnar Nordström) - besides Berlin, the most important center of relativistic studies in Europe at that time. These documents demonstrate that Einstein's confusion about the meaning of coordinates was not simply an idiosyncratic blunder, but the result of widespread and deep-seated prejudices with which early relativists could not easily dispense. Through the point-coincidence argument, Einstein successfully

\footnotetext{
${ }^{2}$ This correspondence has recently appeared in the second volume of Lorentz's correspondence edited by A.J. Kox, to whom I am deeply grateful for giving me access to this material in advance.
} 
convinced the Leiden group to recognize that the choice of coordinates was completely arbitrary in general relativity. However, it was the Leiden group that pressured Einstein to resist the sirens of the privileged coordinate system in his search for gravitational wave solutions. It has seldom been noticed that, using the point-coincidence argument once again in correspondence with Gustav Mie, Einstein could entirely eliminate the last remnant of the materiality of the coordinate system (Section 6.2). Soon after that, (c) countering Kretschmann's 'triviality objection', Einstein raised the point-coincidence argument to the status of a fundamental selection principle (Section 7). While pre-general-relativistic theories can be always formulated in a way that more than spacetime coincidences were observable, general-relativistic theories are singled out by the fact that such formulations are impossible. Nothing but coincidences are observable in general relativity.

Modern relativists are already used to thinking of coordinates as meaningless parameters and find early 'reification' of the coordinate network hard to fathom. In this sense, the idea of 'coordinate scaffolding' can be probably regarded as an instance of what Gaston Bachelard (1938) has called an 'epistemological obstacle'. ${ }^{3}$ These obstacles are not of a technical (i.e., either mathematical or experimental) nature, but rather of a 'conceptual' one. Once they are overcome, it appears surprising that they might have constituted a hindrance in the first place. To capture this discrepancy, this paper makes large use of original texts and private correspondences in which these prejudices are recorded more or less immediately, when the information obtained post facto, after the 'obstacle' was removed, was not available. Breaking an epistemological obstacle is ultimately a philosophical act, despite being entangled in the technical details of a theory as complex as general relativity, an act that, in Bachelard's nomenclature, creates an 'epistemological rupture'. Indeed, Einstein insisted until the end of his life that, minor new predictions aside, the greatest achievement of general relativity was the 'overcoming' of the concept of inertial frame (Einstein to Besso, Aug. 10, 1954; Speziali 1972, Doc. 210). By showing that physics was still possible without a material reference frame, the point-coincidence argument had a third function, which, moving beyond Bachelard's terminology, might be called an 'epistemological reconciliation', the reestablishment of continuity after the rupture. Previous theories aimed to predict the position of point particles with respect to material scaffolding. However, on closer inspection, what these theories were actually predicting has always been the reciprocal coincidences of two physical systems. Ultimately, had the theory been sufficiently powerful, it would have been able to describe the dynamics of both systems conjointly (Giovanelli 2014).

Modern presentations of the mathematical apparatus of relativity theory start at the end of this process where nothing but a manifold of points is left. They attempt to grasp the nature of actual physical spacetime by adding further geometrical structure (topological, differential, affine, metric, etc.) without ever making essential reference to particular coordinates (Friedman 1983; Earman 1989). From the perspective of this coordinate-free approach, many of the problems discussed in this paper might appear

\footnotetext{
${ }^{3}$ Here, I use Bachelard's terminology with some freedom.
} 
technically trivial (Weatherall 2018). However, for the historically minded philosopher of science, it is within the very process of 'becoming trivial' that most relevant conceptual issues are hidden. As a matter of fact, the history of physics has actually moved in the opposite direction (Norton 2002). It started by introducing coordinates as readings on a material rods-and-clocks network, and it was subsequently pressured to progressively deprive them of any unnecessary structure. These two opposite strategies, the additive and subtractive strategies as they might be called (Norton 1999), complement each other. Often in the history of physics, one needs to first enlarge the space of possibilities (as exemplified by the discovery of non-Euclidean geometries, non-Riemannian geometries, etc.) in order to be able to restrict it once again. As is seldom noticed, the point-coincidence argument played a central role in every relevant step of the process of liberating physics from the prison of the coordinate grid. At every step of the process, it was thinking of measurements in terms of 'coincidences' that allowed Einstein to chip away yet another "remnant of the materiality (physikalischer Gegenständlichkeit)" of space and time (Einstein 1916a, 776).

\section{The materiality of the coordinate system: a proto-version of the point-coincidence argument}

\subsection{Coordinates as readings on rods and clocks}

In January 1911, when Einstein received a call to Prague (Illy 1979) and was on the verge of leaving the University of Zurich, he was invited to give a farewell lecture on relativity at the local Naturforschende Gesellschaft (Einstein 1911b). This lecturein which Einstein used the term 'Relativity Theory' in a title for the first time-turned out to be a good opportunity to make some simple epistemological considerations. In particular, Einstein offered a pedagogical account of the role of the coordinate system in physics, presenting it as an extension of practices common in everyday experience. In a world with the electromagnetic field but without gravitational field, objects that carry no electric charge move in straight lines. Thus, one can measure the strength of various forces in terms of how much they cause charged objects (i.e., objects subject to the forces) to deviate from uniform, straight-line motion. Of course, this claim is meaningless if the successive positions of the moving objects are referred to an arbitrarily moving coordinate system (e.g., one undergoing arbitrary rotation). What does it mean, however, to describe the position of a material point relative to a coordinate system?

In mathematical physics, it is customary to relate things to coordinate systems [...] What is essential in this relating-to-something is the following: when we state anything whatsoever about the location of a point, we always indicate the coincidence of this point with some point of a specific other physical system.

\footnotetext{
4In translating Gegenständlichkeit with 'materiality' rather than with the customary 'objectivity' I follow Darrigol (2015).
} 
If, for example, I choose myself as this material point, and say, 'I am at this location in this hall,' then I have brought myself into spatial coincidence with a certain point of this hall, or rather, I have asserted this coincidence. This is done in mathematical physics by using three numbers, the so-called coordinates, to indicate with which points of the rigid system, called the coordinate system, the point whose location is to be described coincides (Einstein 1911b, 2; my emphasis).

At this point, the use of the term 'coincidence' is still an isolated instance in Einstein's writings. Einstein seems to have introduced it merely to illustrate that, in his view, 'space' did not play for the physicist the role of an independent metaphysical entity but is embodied in a concrete, material scaffolding of some sort.

Ultimately, Einstein pointed out, to establish where an object is in space means to specify the 'coincidence' of a point of the object with the point of another physical system, a 'body of reference' (Bezugskörper); it might be the surface of the Earth, the city of Zurich, or the walls of the hall in which Einstein was giving his lecture. To communicate which point of the hall one is referring to, one might place there a material body of sufficiently small dimensions, say the podium of the speaker, as a 'label' that makes the point recognizable. However, if one wants to locate a point in the empty space over the podium, no such marks are available. Then, one might erect a bar perpendicular to the podium, one end of which coincides with the podium and the other with the point in question. The length of the bar, measured with a unit rod, allows marking the point in question so as to communicate its position to others. Proceeding in this way, one can progressively discard the specific characteristics and even the presence of a particular rigid body (the Earth, the hall, the podium, etc.).

As a 'body of reference', one might choose three mutually perpendicular rigid material lines issuing from one point, the origin of the coordinate system, which define three planes. We construct perpendiculars to the coordinate planes (Fig. 1) and count how many times a given unit measuring rod can be laid along these perpendiculars, marking off the values of such counting on the scaffolding. Wherever a point may be located, one can always think of a rigid rectangular system of unit rods that eventually coincide with the point under consideration. The position of a point can then be univocally identified by means of three numbers, the so-called 'coordinates' $x, y, z$ that are the results of measurements made by rigid measuring rods,

Fig. 1 From Einstein and Infeld $(1938,212)$

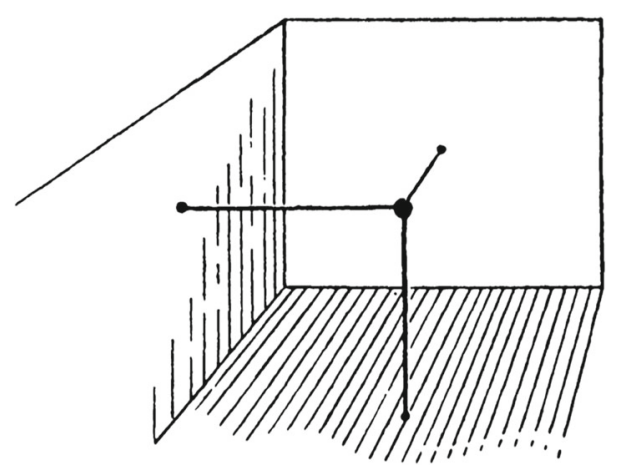


that is, rods having a sequence of marks at regular intervals. The numbering system thus obtained is called a 'Cartesian coordinate system'. We will designate coordinate systems with the letter $K$.

If one wants to describe the motion of the material point, one needs to give the values of $x, y, z$, as three functions of time, that is, one needs to introduce a fourth parameter $t$. A point is at rest relatively to our reference body if these three functions are constant. To define the physical meaning of $t$, one might place clocks at each intersection of rods on the coordinate system $K$. A clock is a system which undergoes a physical process passing periodically through identical phases indicated by some kind of counter, e.g., a fixed-numbered dial. The numerical values of $t$ correspond to the number of such oscillations marked off by the displacement of the clock hands on the dial. We can predict the position, at any time, of a body falling parallel to the $y$-axis ( $x=$ const, $z=$ const), and confirm our prediction by observation. If a graduated rod is placed beside the $y$-axis, we can establish with what mark $y$ on the rod the falling body will coincide at any given moment when the hand of a clock will coincide with the mark $t$. One might, e.g., predict that the coincidence of the particle with the earth is characterized by $t=4$ and $y=0$.

As one can see, Einstein appears to have conceived physical space and time that corresponds to the mathematical space and time as fully 'arithmetized'. A point in space is nothing but a triple of numbers $x, y, z$ and instant of time is nothing but a number $t$ (Norton 2002). It thus becomes possible to treat spatial and temporal relations in a purely algebraic manner. The choice of the parameters $x, y, z, t$ is, in principle, arbitrary. If the geometry of space is Euclidean, although one can resort to other numbering systems (polar, cylindrical coordinates, etc.), ${ }^{5}$ Cartesian coordinates $x, y, z$, are singled out by the fact that, given a unit of length, coordinate numbers directly indicate perpendicular distances from the origin. Formally, it means that it is advisable to chose the coordinate numbers so that the distance $d s$ between any two arbitrarily close points $x, y, z$, and $x+d x, y+d y, z+d z$ satisfies the condition

$$
d s^{2}=d x^{2}+d y^{2}+d z^{2} .
$$

If, for instance, one chooses a different point of origin, or orients the coordinate axes in different directions, to the same point might be assigned a different set of coordinate numbers $x, y, z$, . However, the distance $d s$ between any two close points will be expressed by the same (1). Such substitutions of variables are called 'coordinate transformations', and the equations relating them might be called 'transformation equations'. Euclidean geometry is concerned with the substitutions of spatial coordinates $x, y, z$; classical kinematics considers coordinate transformations involving the time variable $t$ as well.

What Einstein used to call the geometric configuration of a three-dimensional body with respect to $K$ (Einstein 1908, 439; , 28; 1911e, 510) is the totality of distances $s$ between any pair of its points $x, y, z$, as calculated using Eq. 1. The

\footnotetext{
${ }^{5} \mathrm{Cf}$. below footnote 11 .
} 
geometry of the rigid body is the set of substitutions of $x, y, z$, that leaves the geometric configuration of a body unchanged. ${ }^{6}$ All Cartesian coordinate systems $K, K^{\prime}, K^{\prime \prime}$ related by such coordinate transformations are geometrically equivalent. All one can say about them is that each Cartesian coordinate system has a different position or orientation with respect to the other systems. In an analogous way, Einstein labeled the kinematic configuration of a three-dimensional body with respect to $K$ (Einstein 1908, 439; , 28; 1911a, 510) the totality of the distances between any two of its points $x, y, z$, at the same time $t$ as calculated using Eq. 1. The kinematics of the rigid body is the set of substitutions of the coordinates $x, y, z, t$ that leaves the kinematic configuration of a body unchanged. All Cartesian coordinate systems $K, K^{\prime}, K^{\prime \prime}$ related by such transformations are kinematically equivalent. All one can say about them is that each Cartesian coordinate system is moving or at rest with respect to the other systems. If it is tacitly assumed that $t=t^{\prime}=t^{\prime \prime}, \ldots$, then the geometric and kinematic configurations of a three-dimensional body are the same in all kinematically equivalent coordinate systems. ${ }^{7}$

However, the kinematic equivalence of the Cartesian coordinate systems in arbitrary relative motion does not necessarily hold from a dynamical point of view. In particular, it can be shown that, if Newton's laws of motion for point particles can be written in the simplest form with respect to a coordinate system $K$, then they can be written in the same form only in the Cartesian coordinate systems $K^{\prime}, K^{\prime \prime}, K^{\prime \prime \prime}, \ldots$ that are in uniform translational motion with respect to $K$. Such coordinate systems are singled out by the fact that if zero net forces act on a moving particle, its spatial coordinates $x, y, z$, change as a linear function of $t$. True net forces cause particles to deviate from this 'standard' path. Although other coordinate systems can be used, only in such Cartesian coordinate systems can coordinate numbers $x, y, z, t$ be considered as the result of measurements made with 'good' rods and clocks. If one describes the motion of the same force-free particles with respect to a Cartesian coordinate system $K^{\prime}$ coinciding with $K$ at $t=0$, but rotating with angular velocity $\omega$ around the $z$-axis, the particle's coordinates $x, y, z$, appear to change as nonlinear functions of $t$ and extra terms depending on $\omega$ must be added to Newton's laws. Multiplied by the mass of the particles, these terms can be interpreted as fictitious forces deflecting all free particles from their uniform motion. Rods and clocks at rest in these coordinate systems do not reliably measure coordinates differences. Philipp Frank $(1908,192)$ named the set of transformation equations relating dynamically equivalent coordinate systems 'Galilean transformations'. The Galilean transformations constitute the algebraic expression of the Newtonian kinematics of the rigid body in uniform parallel translation.

As is well known, Maxwellian electrodynamics is not compatible with this kinematics. It seemed to be possible to write Maxwell's equations in their simplest form

\footnotetext{
${ }^{6}$ E.g., the geometric configuration of a sphere of radius $r$ centered at the origin of $K$ is given by the equation $x^{2}+y^{2}+x^{2}=r^{2}$. This equation is the same for all other Cartesian coordinate systems however positioned or oriented.

${ }^{7} \mathrm{~A}$ sphere of radius $r$ whose coordinates $x, y, z$, satisfy $x^{2}+y^{2}+x^{2}=r^{2}$ with respect to $K$ will be a sphere with respect to all coordinate systems moving with respect to $K^{\prime}$.
} 
only with respect to one Cartesian coordinate system $K$, the coordinate system with respect to which the velocity of light is $c$, but not with respect to all other coordinate systems moving differently. A coordinate scaffolding is necessary not only for the description of locations but also for the representation of vectors, like the electric and magnetic vector fields $\mathbf{E}(x, y, z)$ and $\mathbf{B}(x, y, z)$. At each of the points $x, y, z$, at the time $t$ one can decompose each field into in its three components $E_{x}, E_{y}, E_{z}$ and $B_{x}, B_{y}, B_{z}$ along the three axes of $K$. Once one has established where the point $x, y, z$, is physically located, one can bring a suitable measuring device in coincidence with that point. It might be a spring balance, so graduated to measure force in an unaccelerated system, provided with an electric charge. The acceleration experienced by the charge in the $x$-direction will bring a pointer on a dial to coincide with a mark; the number one reads is the value of the component $E_{x}$ of the electric field at $x, y, z$, at the time $t$. A similar procedure can be used to measure the components of $\mathbf{B}$. One can then check whether the values of $\mathbf{E}$ and $\mathbf{B}$ predicted by Maxwell's equations at $x, y, z, t$ correspond to the measured ones. If one carries out the transformation laws of the field vectors $\mathbf{E}$ and $\mathbf{B}$ with respect to the Galilean transformations, one can calculate the components of $\mathbf{E}$ and $\mathbf{B}$ with respect to a moving system $K^{\prime}$. The transformed quantities do not obey Maxwell's equations in the same form, with respect to the new coordinate system $K^{\prime}$, i.e., $\mathbf{E}$ and $\mathbf{B}$ are not the same functions of the new coordinates. The fields $E_{x}, E_{y}, E_{z}$ and $B_{x}, B_{y}, B_{z}$ measured with respect to $K$ represented the 'real' state of the ether; the transformed field components $E_{x}^{\prime}, E_{y}^{\prime}, E_{z}^{\prime}$ and $B_{x}^{\prime}, B_{y}^{\prime}, B_{z}^{\prime}$ with respect to a coordinate system $K^{\prime}$ moving in uniform translation with respect to $K$ were deemed as calculational tools.

\subsection{Questioning the physical meaning of coordinates}

However, this theoretical asymmetry between the rigid ether system $K$ and the system $K^{\prime}$ moving in parallel translation with respect to it had no physical counterpart. Einstein (1905) realized that the apparent inconsistency between mechanics and electrodynamics could be removed by challenging an unanalyzed prejudice implicit in the Newtonian kinematics of the rigid body in parallel uniform translation. It could be surmised that the result of rods-and-clocks measurements in two different coordinate systems will assign not only different numbers to $x, y, z$, , but also different numerical values to the time coordinate $t$. In this way, Einstein was able to reshape the traditional relations between the geometry and kinematics of the rigid body. "We obtain the shape of a body moving relative to the system $K$ with respect to $K^{\prime}$ by finding the points of $K^{\prime}$ with which the material points of the moving body coincide at a certain time $t$ of $K^{\prime \prime}$ " (Einstein 1911e, 510). Thus, the assertion about the shape of a moving body acquires quite a complicated meaning since this shape can be ascertained only with the aid of determinations of time. As it turned out, the geometric configuration of a rigid body with respect to $K$ is not necessarily identical to its kinematic configuration with respect to $K^{\prime}$ as it was tacitly assumed in classical kinematics. $^{8}$

\footnotetext{
${ }^{8} \mathrm{~A}$ sphere moving along the $x$-axis whose coordinates satisfy $x^{2}+y^{2}+x^{2}=r^{2}$ with respect to $K$ will be a flattened ellipsoid whose coordinates satisfy $x^{2} / \sqrt{1-\mathbf{v}^{2} / c^{2}}+y^{2}+x^{2}=r^{2}$ with respect to $K^{\prime}$.
} 
A rigid body is defined thereby with respect to a coordinate system $K$, which, in turn, must be regarded as a rigid body. Therefore, Einstein's introduction of a new kinematics of the rigid body implies at the same time a redefinition of what counts as a rigid coordinate system $K$. Einstein could then introduce a new set of linear transformation equations, the famous Lorentz transformations, relating the numerical values of the coordinates $x, y, z, t$ of a point in $K$ and the values $x^{\prime}, y^{\prime}, z^{\prime}, t^{\prime}$ that the same point in $K^{\prime}$ satisfying the condition that the velocity of light in vacuum is expressed by the same numerical value $c$ in the two systems. Once we interpret coordinates $x, y, z, t$ as measurable with identical rods and clocks (which have the same length and run at the same rate when compared with each other at relative rest), the introduction of the Lorentz transformations is not simply a conventional step, but implies predictions concerning the actual behavior of existing physical systems, predictions that can be experimentally verified or falsified, independently of any dynamical laws. However, since coordinates enter in the formulation of all fundamental laws of physics, the Lorentz transformations could be elevated to a criterion to judge well-establish dynamical laws, and a heuristic tool to find new ones (Einstein 1911c, 13). As it turned out, Maxwell's equations happen to already satisfy this condition. The vectors $\left(E_{x}, E_{y}, E_{z}\right)$ and $\left(B_{x}, B_{y}, B_{z}\right)$ play the same role in Maxwell's equations with respect to $K$ as the vectors $\left(E_{x}^{\prime}, E_{y}^{\prime}, E_{z}^{\prime}\right)$ and $\left(B_{x}^{\prime}, B_{y}^{\prime}, B_{z}^{\prime}\right)$ play in Maxwell's equations referred to $K^{\prime}$. Newton's law of motion had to be modified (Giovanelli 2020).

The impact of relativity theory on our conceptions of space and time was indeed deep. In classical mechanics the claim that two occurrences take place 'at the same place in different times' makes no sense unless a rigid 'body of reference' is pointed out. Relativity theory forced us to recognize that the claim that two occurrences take place 'at the same time in different places' also depends on the 'body of reference'. Nevertheless, 'where' and 'when' something occurs were always meant as a physical 'coincidence' with some material, non-accelerated 'body of reference'. Relativity theory ultimately only changed our definition of what counts as as 'good' 'rigid coordinate scaffolding' $K$. As is well known, Einstein (1908) felt that the theoretical asymmetry between a 'good' acceleration-free $K(x, y, z, t)$ and the 'bad' coordinate system $K^{\prime}\left(x^{\prime}, y^{\prime}, z^{\prime}, t^{\prime}\right)$ accelerating with respect to the former was at least questionable from an epistemological point of view. Thus, he sought a more encompassing set of transformation equations that would include Cartesian coordinate systems that are accelerating (and rotating) relative to each other.

However, Einstein soon started to realize that the usual definition of coordinates in terms of cubical scaffolding of rigidly connected rods and clocks had become problematic in uniformly accelerating and rotating coordinate systems. As we have seen, relativity theory was ultimately conceived by Einstein as a new kinematics of the rigid body in uniform parallel translation; but Einstein immediately started to suspect that the development of full relativistic rigid body kinematics, which would include accelerations and rotations, might lead to unexpected consequences (Einstein 1907a; 1907b). Indeed, Max Born (1909) soon showed that in relativity the kinematic definition of a three-dimensional rigid body, even in the simple case of linear uniform 
acceleration, was a delicate matter. Rigidity, which was a kinematic 'property' of a three-dimensional body in the classical theory, turned to be a dynamic 'instruction' of how to apply forces to different points of a body so that the reciprocal distances between the body's points remain constant in the comoving frame. Paul Ehrenfest (1909) immediately pointed out that a 'Born rigid body' could not be put into rotation (Einstein 1911e). Einstein sensed that the question of the rigid body in special relativity might have significant implications for the very definition of the coordinates in accelerated frames (Stachel 1989; Maltese and Orlando 1995).

After some years of work on the quantum problem Einstein (1909a, b, 1910a, c), in June 1911, while in Prague, Einstein (1911b) got back to the problem of gravitation. A few years earlier, Einstein (1908) had singled out the well-known empirical fact that the ratio between gravitational 'charge' and inertial mass is constant, in contrast to, say, the ratio of electric charge and inertial mass. Making a particle more massive does not make it more resistant to gravitational attraction since it also increases its gravitational charge. Thus, it becomes impossible to single out a 'good' unaccelerated coordinate system in which force-free particles move uniformly on straight lines. The same reference system can be interpreted, with equal justice, as a 'good' coordinate system $K$ at rest in which there is a (homogeneous) gravitational field directed along the $-z$-axis (with gravitational acceleration $\gamma$ ) or as a noninertial, 'bad' frame $K^{\prime}$ accelerating in the $z$-direction (with acceleration $-\gamma$ ). "If we accept this assumption" "we obtain a principle that possesses great heuristic significance" (Einstein 1911d, 900).

It becomes possible to transfer the results obtained in an accelerated system to a system with a uniform gravitational field. If a clock in an inertial system $K(x, y, z)$ reads time $t$, by a coordinate transformation one can infer that the same clock would read time $t^{\prime}=t\left(1+\gamma x^{\prime} / c^{2}\right)$ in a coordinate system $K^{\prime}\left(x^{\prime}, y^{\prime}, z^{\prime}\right)$ in uniform acceleration with respect to $K$. However, such a system is indistinguishable from a system at rest in a uniform gravitational field. Thus, one can predict that the time $t^{\prime}$ measured by an identical clock would be $1+\Phi / c^{2}$ times slower if it is placed in an inertial system $K$ at rest in a gravitational field (where $\Phi=x^{\prime} \gamma$ is the gravitational potential). With respect to $K^{\prime}$ not only all free particles but also light rays no longer travel along straight lines, because their $x^{\prime}$-coordinates are no longer linear functions of $t$, etc.; thus the theory predicts that one should observe the same in a uniform gravitational field. In general, if one knows the laws of nature (electrodynamics, thermodynamics, etc.) with respect to a gravitation-free system $K$, then one can, by a mere coordinate transformation, derive the laws relative to $K^{\prime}$ and, thus, the behavior of such a system in a uniform gravitational field.

In February and March, 1912, Einstein (1912a, c) published two papers attempting to find the gravitational field equations for a theory of the static gravitational field with a variable velocity of light. Einstein expressed for the first time in a published paper his concerns regarding the physical significance that one can attribute to coordinates in an accelerating system. He considered an unaccelerated rigid framework of rods $K$, together with a set of suitably synchronized clocks at rest at each point of the framework. He then introduced a system $K^{\prime}\left(x^{\prime}, y^{\prime}, z^{\prime}, t^{\prime}\right)$ accelerating relative 
to the inertial system $K(x, y, z, t)$. Einstein specified that $K^{\prime}$ is linearly accelerated with respect to $K$ in "Born's sense" (Einstein 1912a, 356), that is, in such a way it undergoes a rigid motion, in spite of not being a rigid body (Born 1909). In such a system, differences of spatial coordinates $x, y, z$, can still be measured by Euclidean rods at relative rest with respect to $K^{\prime}$; however, one has to abandon the assumption that clocks directly measure the time coordinate $t$.

However, a Born rigid body cannot be put into rotation without stresses, deformations which will be different for different materials. Therefore the rigid scaffolding used in classical mechanics and relativity theory cannot be used as a model for an accelerated frame of reference in the theory of gravitation. Euclidean geometry "most probably does not hold in a uniformly rotating system" (Einstein 1912a, 356; see Kaluza 1910). If the coordinate system $K^{\prime}\left(x^{\prime}, y^{\prime}, z^{\prime}\right)$ rotates around the axis $z=z^{\prime}$ with respect to $K(x, y, z)$, a rigid unit rod with the same length in every position and every orientation would not directly measure spatial coordinate differences $x^{\prime}, y^{\prime}, z^{\prime}$. Nevertheless, Einstein provisionally considered the interpretation of the coordinate system as a rigid material scaffolding as to be "permitted despite the fact that, according to the theory of relativity, the rigid body cannot possess real existence" (Einstein 1912a, 356). In general, stresses always emerge if the motion is non-Bornian (Herglotz 1911). However, at least in the case of linear acceleration, following Born's instructions, one can imagine "the rigid measuring body being replaced by a great number of nonrigid bodies arranged in a row in such a manner that they do not exert any pressure on each other in that each is supported separately" (Einstein 1912a, 356). This way, since distances do not change for the comoving observer, one can still resort to the direct definition of spatial coordinates.

In a subsequent paper that was received in March of the same year (Einstein 1912c), Einstein investigated the influence of the static gravitational field on the electromagnetic field. He started from Maxwell's equations, which hold relative to a nonaccelerated coordinate system $K(x, y, z, t)$. "The physical meaning of the quantities appearing in these equations is a perfectly determinate one" (Einstein 1912c, 150), he wrote. In such a system, $x, y, z$, are measured by rods laid along the rigid system $K$ and $t$ is measured by identically constituted clocks arranged at rest at the points of the system $K$. The vector fields $\mathbf{E}$ and $\mathbf{B}$ might be measured by spring balances and so on. One can obtain the form of Maxwell's equations in a system $K^{\prime}\left(x^{\prime}, y^{\prime}, z^{\prime}, t^{\prime}\right)$ uniformly accelerated (in Born's sense), by applying the transformation laws for $\mathbf{E}$ and $\mathbf{B}$ corresponding to the coordinate transformations for a system accelerating along the $x$-axis, so that $x^{\prime}=x+\mathbf{a} c / 2 t^{2}$, where $\mathbf{a}$ is the acceleration. According to the equivalence hypothesis, these transformed equations also determined how the electromagnetic processes occur in a static gravitational field. In principle, if this theory must have an empirical content, the transformed quantities should still have a defined physical meaning (Einstein 1912b, 446). For example, one can measure the components of $\mathbf{E}^{\prime}$ with respect to $K^{\prime}$ with a 'pocket' spring balance that can be transported to the point $x^{\prime}, y^{\prime}, z^{\prime}$ in $K^{\prime}$ with different gravitational potentials and check whether the predictions of the theory concerning the components of $\mathbf{E}$ and $\mathbf{B}$ are correct (Einstein 1912b, 447). 
This provisional compromise did not last long. Einstein soon realized that, in the general case of nonuniform gravitational fields, the statement of the principle of equivalence "can be valid only for infinitely small fields"; thus, "Born's accel. finite system cannot be considered a static gravitational field" (Einstein to Ehrenfest, Jun. 20, 1912; CPAE, Vol. 5, Doc. 409), as Einstein initially thought. In a further paper (Einstein 1912b), by addressing some objections of Max Abraham (1912a), Einstein started to realize that the role of coordinates in physics as readings on rods and clocks had to be completely abandoned. Following Abraham (1912b), Einstein implemented for the first time (Minkowski 1909) formalism, in which the three-dimensional kinematics was translated into four-dimensional geometry (Renn 2007b). The spatial coordinates $x, y, z$, and the time coordinate $t$ were treated on an equal footing. In analogy with Eq. 1, the interval $d s$ between two infinitesimally closed spacetime points can be expressed as function of the spacetime coordinates as:

$$
d s^{2}=d x^{2}+d y^{2}+d z^{2}-c^{2} d t^{2},
$$

where $c(x, y, z)$ is variable and plays the role of a scalar potential of the gravitational field. ${ }^{9}$ The equivalence principle opens up the possibility that the equations of the relativity theory that would also include gravitation may also be invariant with respect to acceleration and rotation transformations, that is, to nonlinear transformations. Einstein might have realized that, just as the coefficient $c$ of the time coordinate is not constant in a linearly accelerating system, transforming the line element (2) to rotating Cartesian spatial coordinates depends on coefficients that involve the angular frequency of rotation $\omega$. This dependency has the consequence that also spatial coordinates do not directly correspond to rods-and-clocks readings. Already in a static gravitational field, Einstein commented that "the spacetime coordinates will lose their simple physical meaning" (Einstein 1912b, 1064; my emphasis).

Einstein, like most physicists, considered the very notion of a massive rigid 'coordinate scaffolding' an indefeasible condition for the possibility of physics. When one claims 'a particle will be at $x, y, z$, at $t$ ', the latter numbers must be translated into something like 'the particle coincides with the southeast corner of the lab at 5 o'clock'. If an interpretation of this sort is not possible, the prediction does not seem to have any verifiable meaning. However, the 'materiality' of the coordinate scaffolding was put in serious danger by his theory of gravitation. This tension is clearly recognizable in a little-known remark appended to a manuscript on special relativity on which Einstein worked in Prague and Zurich starting from 1912 (Rowe 2008):

One might ask whether such fastidious physical definitions for the time and space coordinates are really necessary, that is whether it is really necessary to burden the gentle and airy concepts of space and time with heavy rigid bodies and clocks. In my opinion, it is not necessary, but it is advantageous to do so. It is also possible to regard the $x, y, z, t$ as mere mathematical variables (parameters), which have only a meaning in facilitating the formulation of physical

\footnotetext{
${ }^{9}$ The variability of $c$ is actually only a coordinate effect.
} 
laws. The laws formulated with the help of such parameters then have content only in so far as these parameters can be eliminated from several of them. This method of treatment does not alter the law by introducing arbitrary functions of these variables in the same way as $x, y, z, t$. Our definitions for these coordinates can be regarded as a convenient method of elimination. However, I believe that the considerations and definitions given here are sufficient for space and time only as long as one forgoes the introduction of gravitation into the system of relativity (Einstein 1912; fn. 60; my emphasis)

Already in this early passage, Einstein stated clearly that there are two possible ways to consider coordinates: (a) coordinates have physical significance, informing us of the measurable space and time intervals; (b) coordinates are just parameters, catalog numbers that serve only to distinguish a point from another (Westman and Sonego 2008). As Einstein conceded (for which see also Einstein , 25-26; fn. 1), all physical theories, including mechanics and electrodynamics, in principle allow for both interpretations of the coordinates. It is possible to express the equations of physics using polar or cylindrical coordinates or other arbitrary parametrizations. However, in general dynamical laws cannot be expressed in the simplest form in such systems. ${ }^{10}$ As it turns out, only those coordinate systems for which these laws assume their simplest form happen to be directly related to space and time measurements made by rods and clocks (a). In that case, the coordinate system can be replaced by the material coordinate scaffolding. Using (a) thus has a clear advantage over (b). If one follows (a), expressions like $\mathbf{E}(x, y, z, t)$ and $\mathbf{B}(x, y, z, t)$ are not just a mathematical functions, but physically significant quantities. As Einstein seemed to allude at the end of the passage just quoted, the universal nature of gravitation made the interpretation (a) impossible. The influence of, say, the electromagnetic field on the reference body can be made as small as one wants; on the contrary the action of gravitation on the reference body can never be neglected. A nonaccelerated reference system $K$ behaves in the presence of a homogeneous gravitational field in the same way as if it were a suitably chosen accelerated reference frame $K^{\prime}$. It becomes impossible to establish whether $K$ is a 'good' coordinate system in which nonaccelerated rods and clocks at rest reliably read coordinate differences or a 'bad' one $K^{\prime}$ accelerating in the opposite direction, in which coordinate numbers do not directly denote distances. Thus, one lacks a direct procedure to 'eliminate the parameters' and substitute them with physically meaningful numbers.

\subsection{Coordinates as physically meaningless parameters}

By 1912, Einstein had reached the worrying conclusion that the meaning of coordinates as directly determined by measurements with rods and clocks had to be abandoned in a theory of gravitation. The further steps from a scalar to a tensor theory

\footnotetext{
${ }^{10}$ E.g., extra terms appear in the acceleration of a particle when writing Newton's laws of motion in polar coordinates.
} 
of gravity are usually reconstructed relying on Einstein's later recollections (Einstein 1923 ; 1933) but remain little documented by direct textual evidence. Einstein, at some point, while still in Prague, must have seen the analogy between Minkowski's line element $d s^{2}=-d x_{1}^{2}-d x_{2}^{2}-d x_{3}^{2}+d x_{4}^{2}$ (setting $x_{4}=c t$ ) in an accelerated or rotational coordinate system and the line element in Gauss's theory of surfaces, the quadratic differential form:

$$
d s^{2}=E d p^{2}+F d p d q+G d q^{2} .
$$

Einstein might have learned about it in Carl Friedrich's (1897) lectures that he had attended at the ETH (Reich 1994, 163ff.). In Gauss's approach, one can cover a curved surface using two families of nonintersecting curves so that, within each family, each curve is distinguished by the parameters $p(p=1, p=2$, etc.) and $q$ ( $q=1, q=2$, etc.). If these two families are selected to be infinitely dense, one can in principle label any point on the surface through their intersections $p=$ const and $q=$ const. For example, the point $(5,3)$ is where the coordinate lines $p=5, q=3$ intersect. If one is dealing with a plane surface, one can always introduce a regular network in which, given a unit of measure, these numbers directly represent actual lengths from the origin $p=0, q=0$ using Eq. 1. However, in the general case, one needs to know the three coefficients $E, F$, and $G$ at every point $(p, q)$ in order to convert small coordinate differences $d q$ and $d p$ into actual distances $d s$ on the surface. ${ }^{11}$ Different surfaces are represented by different quadratic forms; however, the converse is not true; the same surface can be covered with different grids of lines. In order to decide whether two quadratic forms determine the same surface, one needs to calculate the Gaussian curvature from $E, F$, and $G$ and its first and second derivatives. Nevertheless, Einstein did not seem to be interested in Eq. 3 inasmuch as it

\footnotetext{
${ }^{11}$ In Cartesian coordinates, once the origin and a unit of measure have been fixed, these numbers also denote distances. Thus, the small distance $d s$ between any two neighboring points can be directly calculated from the difference between their coordinates $d s^{2}=d q^{2}+d p^{2}(E=1, F=0, G=1)$. This is not the case in other coordinate systems, like polar coordinates $p=r, q=\vartheta$ on a flat surface, in which the line element takes the form $d s^{2}=d r^{2}+r^{2} d \vartheta^{2}\left(E=1, F=0, G=r^{2} \sin ^{2}\right)$. Equal increments in radial distance $d r$ correspond to real distances $d s^{2}=d r^{2}$ from the center; however, small increments in angular coordinates $d \vartheta$ correspond to $1 / \sqrt{G}$ real distances. On a flat surface, one can always switch back to Cartesian coordinates, in which the parameters $p, q$ have a direct metrical meaning. However, this is not true in the general case. The fact that a sphere cannot be projected onto a plane without distortion can be expressed analytically by the fact that it is impossible to convert the quadratic differential form that holds on a sphere into the one holding on a plane through a mere transformation of the independent variables. On a sphere, it is suitable to introduce coordinates $p=\vartheta$ and $q=\varphi$, which are the customary latitude (counted from the equator) and longitude (counted from the meridian of Greenwich) measured in radians. Constant angular increments $d \vartheta$ do not denote constant distance increments $d s$. Increments $d s$ are related to coordinate increments by $d s^{2}=R^{2} d \vartheta^{2}+R^{2} \cos ^{2} \vartheta d \varphi^{2}$, where $R$ is the radius of the sphere, which, without loss of generality, can be set equal to $1\left(E=1, G=\cos ^{2} \vartheta, F=0\right)$. While differences in longitude $d \vartheta$ correspond to actual distances $d s$, differences in latitude $d \varphi$ correspond to $1 / \sqrt{G}$ real differences $d s$. The distance between any two arbitrarily distant points can be measured by the minimal number of links of a chain of $d s$ joining the two points.
} 
defines the intrinsic geometrical structure of the surface, but because it offers a sort of 'algorithm' to convert meaningless parameters into measurable distances. ${ }^{12}$

After getting back to Zurich in August 1912, with the help of Marcel Grossmann (Sauer 2015), Einstein became familiar with Ricci and Levi-Civita's 'absolute differential calculus' (Levi-Civita and Ricci-Curbastro 1900; see Reich 1994), which provides a general method to deal with quadratic differential forms like Gauss's line element. At the same time, he realized the fundamental importance of the 'world tensor' $T_{\mu \nu}$ to represent the source of the gravitational field (Norton 1992). Just like someone who, knowing only electrostatic and magnetostatic phenomena, attempts to develop a field theory encompassing all electromagnetic phenomena, Einstein aimed to develop a field theory of gravitation starting only from Poisson's equations valid for static fields and slow-moving particles. The Zurich Notebook (CPAE, Vol. 4, Doc. 10) documents how Einstein was able to move from Newton's scalar theory of gravitation to one based on a quadratic differential form:

$$
d s^{2}=\sum G_{\mu \nu} d x_{\mu} d x_{\nu},
$$

where $d s$ is a scalar. $G_{\mu \nu}$ was later written as $g_{\mu \nu}$, a notation that we will adopt from now on. It indicates a tensor that can be written as a matrix with four rows and four columns, or 16 components in total, as $\mu$ and $v$ range from 1 to 4 . The number of independent components reduce to 10 if $g_{\mu \nu}=g_{\nu \mu}$. Einstein's struggles (Renn

\footnotetext{
${ }^{12}$ Cartography provides an excellent analogy (Janssen 1998) to which we will return throughout the paper. Indeed, most of the confusions about coordinates analyzed in this paper make sense if we make the hypothesis that physicists thought in terms of map projections, rather than in terms of the intrinsic structure of surfaces. Let us say that one wants to draw a flat map of the Earth. Since a map of the earth at $1: 1$ scale is impossible, one substitutes the earth with a globe, which is an exact replica of the earth in scale. The problem of a perfect map is expressed by the demand that one should be able to establish a one-to-one correspondence between the two surfaces such that $d s^{2}$ of the globe and $d s^{2}$ of the map coincide in corresponding points. One can map a sufficiently small portion of the globe, say a city, without distortions on a flat paper (or onto a surface developable into a plane, such as a cylinder or a cone) covered by a Cartesian coordinate grid $x, y$. If one measures the coordinate difference $d x$ between two streets on the map parallel to the the vertical $y$-axis with a ruler, the distance measured will correspond to the actual distance between the two cities on the globe. The same procedure is not possible for maps of larger portions of the globe in which distortions necessarily occur. In translating latitude and longitude into Cartesian coordinates by some projection function $x=f(\vartheta, \varphi), y=f(\vartheta, \varphi)$, one can see that equal coordinate differences on the map do not correspond to equal distances on the surface. In order to recover real distances $d s$ between two close cities on the globe from their coordinates on the map $x, y$ and $x+d x, y+d y$, one needs to know the three functions $E, F, G$ at every point of the map. Let us consider a cylindrical projection. It can be shown that, in such projection, $E=\left(1-\frac{y^{2}}{R^{2}}\right), G=\left(1-\frac{y^{2}}{R^{2}}\right)^{-1}, F=0$. The functions $E, F, G$ are the 'legend' of the map. If one measures equal distances $d x=1$ along the $x$-axis on the map, one will get real distances on the globe $d s=\sqrt{E} d p$, which are $\neq 1$. The results become more distorted toward the poles. In a stereographic projection, the map legend is different: $E^{\prime}=G^{\prime}=4\left(1+\frac{x^{2}}{R^{2}}+\frac{y^{2}}{R^{2}}\right)^{-2}, F^{\prime}=0$. As one can see, the same coordinate difference $d x=1$ measured on the projection plane is multiplied by a different factor: $d s=\sqrt{E^{\prime}} d x$. Therefore, the same coordinate differences $d x$ and $d y$ might correspond to vastly different real differences on the globe, depending on the projection used. Thus, without knowing the 'map legend'- that is, the functions $E, F, G$-it is impossible to predict the actual distance $d s$ between two close cities from the small differences $d x$ and $d y$ of their coordinates on the map (see footnote 14). Needless to say, the relation is actually reversed: the same $d s$ corresponds to different $d x, d y$ in different maps projections; $d x, d y$ are meaningless without a legend.
} 
and Sauer 2007) finally led to a two-part Entwurf (that is, 'Outline') of a theory of relativity and gravitation coauthored with Grossmann, which was published in June 1913 (Einstein and Grossmann 1913).

In the physics part of the Entwurf paper, Einstein formulated the equivalence principle in the following way. Introduce a system of reference $K(\mathrm{x}, \mathrm{y}, \mathrm{z}, \mathrm{t})$ in a sufficiently small region of spacetime. With respect to this frame, a body moves uniformly in a straight line according to the equation $\delta\left(\int d s\right)=0$, whereas

$$
d s^{2}=-d x_{1}^{2}-d x_{2}^{2}-d x_{3}^{2}+d x_{4}^{2},
$$

where the coefficient $c$ in $x_{4}=c t$ is the constant velocity of light. Einstein conceived a transformation from the inertial frame $K$ to the accelerated frame $K^{\prime}$ as a coordinate transformation. Thus, if one describes the motion of a body from the perspective of a system $K^{\prime}\left(x^{\prime}, y^{\prime}, z^{\prime}, t^{\prime}\right)$ moving with acceleration a in the direction along $x$, one has to introduce new coordinates $x^{\prime}, y^{\prime}, z^{\prime}, t^{\prime}$, which are nonlinear functions of $x, y, z, t$. The simplest transformation leads to the line element

$$
d s^{2}=-d x_{1}^{2}-d x_{2}^{2}-d x_{3}^{2}+x_{1}^{2} \mathbf{a}^{2} x_{4}^{2} .
$$

In this system, all free objects and light rays appear to be uniformly accelerated in a direction opposite that of the acceleration of $K^{\prime}$ so that their free motion according to $\delta\left(\int d s\right)=0$ will appear as curvilinear with respect to $K$.

In Minkowski's formalism, the difference between $K$ and $K^{\prime}$ is easy to spot. In $K$, given an appropriate choice of the coordinates, the matrix $g_{\mu \nu}$ has constant values, with $-1,-1,-1$ and $c^{2}$ as diagonal terms, and all other terms are equal to 0 . In $K^{\prime}, g_{\mu \nu}$ are functions of the coordinates; in particular, $g_{44}=\mathbf{a}^{2} x_{1}^{2}$ is a function of $x_{1}$. In pre-general-relativistic physics, the Cartesian coordinate system $K$ would be regarded as a 'good' coordinate system, with respect to which light rays and free particles move on straight lines; the non-Cartesian system $K^{\prime}$ would be regarded as a 'bad' coordinate system (as the appearance of fictitious forces reveals), and the curvilinear nature of paths of light rays and free particles with respect to $K^{\prime}$ had no physical meaning. The equivalence principle eliminates the asymmetry between 'good' and 'bad' coordinate systems. A linearly accelerated coordinate system $K^{\prime}$ is indistinguishable from an inertial system $K$ with a gravitational field. Thus, Einstein drew the following conclusion: The variability of $g_{\mu \nu}$ with respect to the coordinates $x_{1}, x_{2}, x_{3}, x_{4}$, which mathematically tells us that we are using non-Cartesian coordinates, could be identified physically with the presence of a homogeneous gravitational field.

This suggested that the components $g_{\mu \nu}$ could take the place of the single Newtonian gravitational potential $\varphi$ also in the general case, in which non-constant $g_{\mu \nu}$ cannot be transformed away by a simple coordinate transformation. Einstein arrived at the view that the gravitational field is characterized by 10 spacetime functions $g_{\mu \nu}$, which acquire a double meaning. On the one hand, $g_{\mu \nu}$ are the potential of a physical field, comparable to the electromagnetic field, which assumes particular values at certain points identified by their coordinates $x_{\nu}$. At the same time, $g_{\mu \nu}$ are geometrical quantities, conversion factors that relate coordinate differences $d x_{v}$ and real distances $d s$ according to Eq. 4. Consequently, the direct relationships between the coordinate differences $x_{1}, x_{2}, x_{3}, x_{4}$ and rods-and-clocks measurements is lost: 
From the foregoing, one can already infer that there cannot exist relationships between the space-time coordinates $x_{1}, x_{2}, x_{3}, x_{4}$ and the results of measurements obtainable by means of measuring rods and clocks that would be as simple as those in the old relativity theory. With regard to time, this has already found to be true in the case of the static gravitational field. The question therefore arises, what is the physical meaning (measurability in principle) of the coordinates $x_{1}, x_{2}, x_{3}, x_{4}[\ldots]$ From this one sees that, for given $d x_{1}, d x_{2}, d x_{3}, d x_{4}$, the natural distance that corresponds to these differentials can be determined only if one knows the quantities $g_{\mu \nu}$ that determine the gravitational field. This can also be expressed in the following way: the gravitational field influences the measuring bodies and clocks in a determinate manner (Einstein and Grossmann 1913, 8)

In previous theories, there was a privileged class of rectangular coordinate systems such that, knowing the difference $d x_{1}, d x_{2}, d x_{3}, d x_{4}$ between the coordinates of any two points, one could predict the distance $d s$ between them as it would be measured by nonaccelerated rods and clocks, and vice versa. In the Entwurf Theory, by contrast, once one knows the coordinates of two spacetime points $x_{1}, x_{2}, x_{3}, x_{4}$ and $x_{1}+d x_{1}, x_{2}+d x_{2}, x_{3}+d x_{3}, x_{4}+d x_{4}$, without knowing the functions $g_{\mu \nu}$, one cannot predict the rods-and-clocks distance $d s$. However, in order to determine $g_{\mu \nu}$, one must already know the measured distance $d s$ between two points of coordinates $x_{1}, x_{2}, x_{3}, x_{4}$ and $x_{1}+d x_{1}, x_{2}+d x_{2}, x_{3}+d x_{3}, x_{4}+d x_{4}$. There seems to be a circle. Einstein famously broke the circle by relying on the fact that, in a sufficiently small region of spacetime, one can always introduce a coordinate system in which $g_{\mu \nu}$ can be taken to be constant in the first approximation, that is, in which special relativity holds. 13

One can switch from the given arbitrary coordinate system to a rectangular coordinate system and determine the length $d s^{2}$ using rods and clocks at relative rest. This is called the 'natural' four-dimensional interval, as opposed to the 'coordinate' interval. This natural length is, by definition, a scalar and can be set equal to \pm 1 , up to an arbitrary choice of unit, and directly measured with rods and clocks. One can set, say, $n_{1}$ spacings between the atoms of a rock-salt crystal as $=-1$ and $n_{2}$ electromagnetic wave crests emitted by a cadmium atom as $=1$. One can then switch back to the originally given coordinate system. Since the numerical value of $d s^{2}$ per definition does not change, from Eq. 4, one can read off $g_{\mu \nu}$ for known $d s^{2}$ and $d x_{v}$. Over larger regions of spacetime, it might be impossible to introduce a coordinate system in which coordinate differences denote actual distances. In the general case, $g_{\mu \nu}$ can be measured as the numbers by which coordinate differences have to be multiplied so that $d s^{2}=1$ in every position and in every orientation. For example, a unit rod laid along the $x_{1}$-axis $\left(d x_{2}=0, d x_{3}=0, d x_{4}=0\right)$ will measure $-1=\sqrt{g_{11}} d x_{1}$; a unit clock at rest will measure $1=\sqrt{g_{44}} d x_{4}$. This measurement procedure is meaningful under the condition that the ratio $n_{1} / n_{2}$ is not influenced by the gravitational field so that equally constructed unit rods and unit clocks always measure the same

\footnotetext{
${ }^{13}$ This is usually taken as a stronger version of the equivalence principle. For the different meanings of the equivalence principle, see Lehmkuhl (2019).
} 
$\pm d s=1$. If this is the case, we can determine $g_{11}=-d s^{2} / d x_{1}^{2}, g_{44}=d s^{2} / d x_{4}^{2}$ and in general all values of the $g_{\mu \nu}$.

The key to Einstein's success in the electrodynamics of moving bodies was his careful analysis of the procedure through which physics, using rods and clocks, gives physical meaning to kinematic variables before any dynamics. However, the introduction of gravitation forced Einstein to 'unlearn' precisely the strategy which had led to him striking success a decade earlier (Stachel 1993). Rods and clocks, the same instruments that serve to measure distances and time intervals, also serve to measure the gravitational field $g_{\mu \nu}$. Since the quantities $g_{\mu \nu}$ enter into this relationship, the coordinates themselves have no independent physical meaning and are reduced to mere numbers. The fact that coordinate parameters, beside serving as labels, also measure distances turned out to be an accident. As Einstein soon came to realize, the kinematic variables $x_{1}, x_{2}, x_{3}, x_{4}$ and the dynamical variables $g_{\mu \nu}$ became entangled into an inextricable knot. As with any other field theory, general relativity aims to predict the components of the gravitational field $g_{\mu \nu}$ at a point $x_{1}, x_{2}, x_{3}, x_{4}$. However, $g_{\mu \nu}$ are also the 'measurement' field that allows calculating the distance of $x_{1}, x_{2}, x_{3}, x_{4}$ with respect to any other point. Einstein struggled for several years to make peace with the fact that one does not know where $x_{1}, x_{2}, x_{3}, x_{4}$ is before the $g_{\mu \nu}$ field is introduced. However, as we shall see, Einstein would need at least three more years to realize that the location of $x_{1}, x_{2}, x_{3}, x_{4}$ is lost again after the $g_{\mu \nu}$ field has been removed. ${ }^{14}$

\section{The (nearly) last remnant of materiality of coordinate system}

\subsection{From the besso-einstein argument to the $\$ 12$ argument}

In the Entwurf paper, Einstein and Grossmann (1913) were able to show that the equation of motion of a mass point in a gravitational field could be written in a coordinate-independent way, without introducing an otherwise nonaccessible privileged class of coordinate systems. However, they were not able to put forward a set of field equations with these covariance properties. In the Zurich notebook, we can follow in great detail Einstein's tentative search for of a set of differential equations connecting the source $T_{\mu \nu}$ and with a second-rank tensor $\Gamma_{\mu \nu}$ depending on $g_{\mu \nu}$ at most up to its second derivatives:

$$
\Gamma_{\mu \nu}=\kappa T_{\mu \nu} .
$$

\footnotetext{
${ }^{14}$ As a comparison, one can think of the coordinates $x, y$ on a map, the legend of which has become unreadable. If one knows that, according to a map, a treasure is buried at $x=45, y=85$ in the middle of the ocean (where no other 'marks' are available if not coordinate numbers), there would be no way to find the latitude and longitude $p, q$ of the treasure on the globe. The numbers $x, y$ are meaningless without the map legend. Thus, independently of the coefficients $E, F$, and $G$, the point $x, y$ on the projection plane is, so to speak, nowhere (see footnote 12). In general relativity, difficulties emerge from the fact that the $g_{\mu \nu}$ are both the map legend (the metric tensor) and the treasure to be found (the gravitational field). Cf. footnote 20 .
} 
The static limit of these equations was supposed to yield Poisson's equation with a single potential, as the static limit of Maxwell's theory yields the Coulomb law. In other terms, the field equations must be reduced to the case in which only the component $g_{44}$ of the gravitational field is variable, that is, a function of spatial Euclidean coordinates. To obtain this result, Einstein started from an expression in which $\Gamma_{\mu \nu}$ is a two-index contraction of the Riemann tensor. ${ }^{15}$ These equations hold in all coordinate systems, whereas Poisson's equation does not. Thus, Einstein needed to impose some coordinate requirement to eliminate unwanted terms and yield only a single potential. In particular, as a modern relativist would do (Norton 1984), he considered the requirement

$$
g^{\mu \nu}\left\{\begin{array}{c}
\alpha \\
\mu \nu
\end{array}\right\}=0,
$$

where the curly bracket is the Christoffel symbol of the second kind. ${ }^{16}$ These kinds of coordinate requirements, besides ensuring that the field equations reduce to their Newtonian limit, were also supposed to guarantee energy-momentum conservation, at the same time leaving enough freedom to include accelerating systems. Einstein seemed to consider coordinate requirements of this sort as a general restriction (Janssen and Renn 2007) on the class of allowable coordinate system introduced once and for all and not simply as a condition imposed provisionally to solve a particular problem. ${ }^{17}$ Therefore, all attempts to proceed in this way lead to considerable difficulties. Switching from the mathematical to the physical strategy (Janssen and Renn 2007), Einstein decided to settle with a set of field equations valid for arbitrary but linear transformations. Thus, ultimately, Einstein and Grossmann were not able to cast the Entwurf field equations in a form that would allow for arbitrary substitutions of the independent variables. Nevertheless, Einstein was convinced that he was on the right track. "The conviction to which I have slowly struggled through is that there are no preferred coordinate systems of any kind," Einstein wrote to Ehrenfest. "However, I have only partially succeeded, even formally, in reaching this standpoint" (Einstein to Ehrenfest, May 28, 1913; CPAE, Vol. 5, Doc. 441).

In June 1913, just around the time the Entwurf Theory appeared in print, Einstein, with the help of Besso, had already started applying the new theory to calculate the gravitational field of the Sun using a weak field approximation, as documented in a manuscript found among Besso's papers after his death (CPAE, Vol. 4, Doc. 14). Einstein and Besso aimed to calculate deviations $\gamma_{\mu \nu}$ from Minkowski's values $\delta_{\mu \nu}$ $\left(\delta_{\mu \nu}=1\right.$ for $\mu=v$ and $\delta_{\mu \nu}=0$ for $\left.\mu \neq v\right)$ so that $g_{\mu v}=-\delta_{\mu \nu}+\gamma_{\mu \nu}$. ${ }^{18}$ Thus,

\footnotetext{
${ }^{15}$ This is the so-called Ricci tensor; see eg., Janssen and Renn (2015a) for a recent account of Einstein's path toward field equations.

${ }^{16}$ Equation 5 corresponds to the so-called 'harmonic coordinate condition'. In modern notation: $\Gamma_{\mu \nu}^{\alpha} g^{\mu \nu}=$ 0 .

${ }^{17}$ The editors of Renn (2007a) have introduced the illuminating distinction between 'coordinate conditions' and 'coordinate restrictions'. I will use 'coordinate requirements' to indicate the general category, including both coordinate conditions and restrictions (for which see footnote 23). For a different interpretation, see Norton (2005).

${ }^{18}$ For the sake of uniformity, the notation adopted here is different from Einstein's, who used Latin letters for covariant components and Greek letters for contravariant components of tensors.
} 
Einstein believed that it was possible to obtain a good approximation by taking these deviations into consideration, along with their derivatives, only where they appear linearly. Imposing the condition (5), the field equations take the following form:

$$
\square \gamma_{\mu \nu}=-\frac{\partial^{2} \gamma_{\mu \nu}}{\partial x^{2}}-\frac{\partial^{2} \gamma_{\mu \nu}}{\partial y^{2}}-\frac{\partial^{2} \gamma_{\mu \nu}}{\partial z^{2}}+\frac{1}{c^{2}} \frac{\partial^{2} \gamma_{\mu \nu}}{\partial t^{2}}=\kappa T_{\mu \nu},
$$

where $\square$ is the d'Alembertian operator ${ }^{19}$ and $T_{\mu \nu}$ is the stress-energy tensor for a dust of particles (that is, particles which do not directly interact with each other); the field is treated as a static field, with slow particle motion, and $\gamma_{\mu \nu}$ are assumed to vanish at infinity. The $g_{\mu \nu}$ with $\mu$ and $v$ ranging from 1 to 3 were expected to be constant $=-1$, so at a certain instant in time $d x_{4}=0$, a rod would directly measure spatial coordinates and would not be distorted by the Newtonian gravitational field. By contrast, a clock at rest in such coordinate system $\left(d x_{1}=d x_{2}=d x_{3}=0\right)$ measures $d \tau^{2}=g_{44} d x_{4}^{2}$; that is, the greater the masses arrayed in its vicinity, the slower the clock runs. The approximate value of the component $g_{44}$ was calculated using the Entwurf equations in first- and second-order approximations. Once one knows $g_{44}$, one can calculate the path of light rays and the planets' orbits. However, Einstein and Besso were not able to account for Mercury's perihelion anomaly. Thus, these calculations were never published (Janssen 2003).

For our limited purposes, this manuscript is interesting for the following considerations. On [p. 1], Besso writes the matrix of the covariant values of $g_{\mu \nu}$ in first-order approximation:

$$
g_{\mu \nu}=-1(\mu, v=1,2,3), \quad g_{44}=\frac{1}{c_{0}^{2}}\left(1+\frac{A}{r}\right), \text { where } A=\frac{\kappa M}{4 \pi} .
$$

A remark in Besso's hand seems to reveal that Einstein and Besso might have become aware of the problem of the uniqueness of the solution they had obtained, as a remark in Besso's hand reveals: "Is the static gravitational field in Eq. 7 $g_{\mu \nu}=1$ to $3 g_{44}=f(x, y, z)$ a particular one? Or is it the general one expressed in spec. coordinates?" (CPAE, Vol. 4, Doc. 14[p. 16]). Thus, Besso seems to have been somehow puzzled by the fact that $g_{\mu \nu}$ are determined only up to a coordinate transformation, that is, that one introduces different values of the components of the gravitational field by switching to a different coordinate system. For a weak field on a background Cartesian coordinate system, one can introduce an infinitesimal coordinate transformation $x_{v}+\xi_{v}$ (where $\xi_{v}$ is similar in size to $\gamma_{\mu \nu}$ ) and obtain different values for $\gamma_{\mu \nu}^{\prime}=-\delta_{\mu \nu}+\gamma_{\mu \nu}^{\prime}$. On the contrary, Einstein and Besso probably expected that, given initial and boundary conditions, the field equations determine $g_{44}(x, y, z)$ unambiguously (i.e., as a single-valued function), just like how Poisson's equation determines the gravitational potential $\phi(x, y, z)$ in a Cartesian coordinate system.

Besso left Zurich for Gorizia and got back to Zurich around August 1913. Probably during this second visit, he jotted down some notes (the so-called Besso memo) about a discussion that he had with Einstein (Janssen 2007). These notes seem to contain an objection against the very possibility of general covariance of such equa-

\footnotetext{
${ }^{19}$ The d'Alembertian operator is the four-dimensional equivalent of the Laplacian $\nabla^{2}=\frac{\partial^{2} \varphi}{\partial x^{2}}+\frac{\partial^{2} \varphi}{\partial y^{2}}+\frac{\partial^{2} \varphi}{\partial z^{2}}$.
} 
tions. Besso argued that, in the case of a central mass, such as the Sun, surrounded by empty space, due to the arbitrary choice of the coordinate system, the field equations (together with boundary conditions imposed at infinity) do not guarantee a unique $g_{\mu \nu}$-system in the empty region. Besso's notes (see Janssen 2007, 792, fig. 2) seem to reproduce a dialog between him and Einstein:

[Besso:] The requirement of 〈general〉 covariance of the gravitational equations under arbitrary transformations cannot be imposed: if all matter 〈is given〉 were contained in one part of space and for this part of space a coordinate system $\langle$ is given $\rangle$, then outside of it the coordinate system could still 〈essentially $\rangle$ except for boundary conditions be chosen arbitrarily, 〈through which the $g$ arbitrarily〉 so that a unique determinability of the $g$ 's cannot be obtained

[Besso:] It is, however, not necessary that the $g$ themselves are determined uniquely, only the observable phenomena in the gravitation space, e.g., the motion of a material point, must be.

[Einstein:] Of no use, since with $\langle$ the $\rangle$ a solution a motion is also fully given. If in coordinate system 1 , there is a solution $K_{1}$, then this same construct is also a solution in 2, $K_{2} ; K_{2}$ however, also a solution in 1 (Besso Memo p. 2; quoted and translated in Janssen 2007, 819-821)

As Besso pointed out, once one calculates a solution $g_{\mu \nu}=K_{1}$, one could obtain a different solution $g_{\mu \nu}^{\prime}=K_{2}$ by simply introducing a new coordinate system 2 . In a generally covariant theory, this solution is just as good as the first since a coordinate system 2 is just as good as 1 . Besso suggested that this might not be a problem. It is not necessary that the field equations determine $g_{\mu \nu}$ in a unique way; it is only necessary that the theory correctly predicts the observable phenomena, for example, that tomorrow morning, when the Sun is five degrees over the horizon, Venus will be visible at 12 degrees over the horizon (Rovelli 2002). In the second part of the quote, Besso probably wrote down Einstein's critique of Besso's counterargument: the new solution $K_{2}$ in the coordinate system 2 'is also a solution in 1', that is, the components of the solution $K_{2}$ can be calculated as functions of the 1-coordinate numbers. Thus, under the given initial and boundary conditions, the Entwurf field equations do not determine the gravitational field univocally with respect to the same coordinate system 1.

From June to early July, Ehrenfest and his wife came to Zurich from Leiden, where they discussed Einstein's new theory (Yavelov 2002) together with the Finnish physicist Gunnar Nordström $(1912,1913 \mathrm{a}, \mathrm{b})$, who had presented an alternative theory of gravity, which Einstein considered as a serious alternative to the Entwurf Theory. As a letter to Lorentz in August 1913 revealed, at that time, Einstein was still unsatisfied with the field equations of limited covariance. It seemed questionable that all equations of physics could be formulated without reference to a specific coordinate system, except for the field equations that regulate the behavior of $g_{\mu \nu}$. Thus, the theory was like "hanging in the air" (Einstein to Lorentz, Aug. 14, 1913; CPAE, Vol. 5, Doc. 467). At some point, Einstein might have realized that his reply to Besso's 
argument could turn out to be useful. The discovery of the Besso memo (Janssen 2007) revealed that the argument, far from being a refined reflection of the nature of spacetime, was more of a 'the Fox and the Grapes' kind of argument. The generally covariant field equations that he was not able to find were after all not desirable in the first place. Field equations that are valid in all coordinate systems do not uniquely determine the gravitational field (Einstein to Lorentz, Aug. 16, 1913; CPAE, Vol. 5, Doc. 470).

In the printed summary of a lecture delivered in September of 1913 at the 96th annual meeting of the Schweizerische Naturforschende Gesellschaft in Frauenfeld, Einstein pointed out for the first time that it is logically impossible to introduce generally covariant field equations (Einstein 1913a, 8). In a footnote of the printed version of a lecture delivered at the Gesellschaft Deutscher Naturforscher und Ärzte in Vienna a few weeks later, Einstein already hinted that "in the last days" he had found "a proof that such a generally covariant solution to the problem cannot exist at all" (Einstein 1913b, 1257; fn. 2). Since the lecture was published in December, it is hard to say whether this remark was added later. Only in November did Einstein explain to Ludwig Hopf (Einstein to Hopf, Nov. 2, 1913; CPAE, Vol. 5, Doc. 480) that he was now satisfied with his field equations of limited covariance, since generally covariant field equations were actually impossible. As Einstein wrote more explicitly to Ehrenfest in the ensuing weeks, "a unique determination [eindeutige Bestimmung] of the $g_{\mu \nu}$ out of the $T_{\mu \nu}$ " was not possible without a special choice of the coordinate system. According to Einstein, this was "rigorously provable" (Einstein to Ehrenfest, Nov. 15, 1913; CPAE, Vol. 5, Doc. 484). Thus, as he wrote to Ehrenfest, the spacetime variables were completely arbitrary, but one can use the conservation laws for energy and momentum to restrict the admissible coordinate transformations to the linear ones (Einstein to Ehrenfest, Nov. 15, 1913; CPAE, Vol. 5, Doc. 484). The 'good' coordinate systems are, so to speak, tailored to the physical world instead of being given a priori (Einstein to Mach, Dec. 15, 1913; CPAE, Vol. 5, Doc. 497). At that time, the Leiden community started to become one of Einstein's most important 'sounding boards'. Einstein was even thinking about hiring Adriaan Fokker or Johannes Droste as assistants since they both showed "Lorentz's excellent training" (Einstein to Ehrenfest, Nov. 15, 1913; CPAE, Vol. 5, Doc. 484).

As is well known, Einstein would reproduce the argument against general covariance in published writings on four occasions (Einstein and Grossmann 1914a, b; Einstein 1914a, b). Starting from the first published on January 30, 1914, in some 'Comments' added to the reprint of the Entwurf paper (Einstein and Grossmann 1914a), to avoid imposing arbitrary additional boundary conditions at infinity, Einstein might have inverted Besso's argument (Janssen 2007). Instead of an insular matter distribution surrounded by an empty space, Einstein introduced an empty region $L$ (which might stand for Loch, hole) surrounded by matter. In this region, no material process occurs (no electromagnetic field, no particles, etc.); that is, $T_{\mu \nu}$ vanishes. In general, points in space are identifiable using some material element that one can use to 'mark' the place in question. However, in empty space, points can 
be identified only by their coordinate numbers. ${ }^{20}$ Given a solution $g_{\mu \nu}$ of the field equations within $L$, the general covariance of the equations allows us to introduce a new coordinate system $x_{\mu}^{\prime}$ so that $x_{v}=x_{\mu}^{\prime}$ at the boundary of $L$, but $x_{v} \neq x_{\mu}^{\prime}$ inside of it (Einstein and Grossmann 1914a, 260). One now relates everything to this new primed system, in which matter outside of $L$ is represented by $T_{\mu \nu}^{\prime}=T_{\mu \nu}{ }^{21}$ and the gravitational field inside of $L$ by $g_{\mu \nu}^{\prime} \neq g_{\mu \nu}$. Thus,

$$
\begin{gathered}
T_{\mu \nu}^{\prime}=T_{\mu \nu} \quad \text { outside of } L \\
\text { does not imply } \\
g_{\mu \nu}^{\prime}=g_{\mu \nu} \quad \text { inside of } L .
\end{gathered}
$$

In other terms, it is possible that "more than one system of $\left[g_{\mu \nu}\right]$ pertains to the system $\left[T_{\mu \nu}\right]$ " (Einstein and Grossmann 1914a, 260; my emphasis). In order to achieve a unique determination of $g_{\mu \nu}$ (gravitational field) by $T_{\mu \nu}$ (matter), Einstein concluded that one has to restrict the choice of the coordinate system.

Einstein's argument against general covariance of the field equations appeared for quite a long time based on a trivial misunderstanding. Of course, it is not surprising that the components $g_{\mu \nu}$ change in a definite manner as the coordinates $x_{v}$ are changed to $x_{v}^{\prime}$, for example, by switching from Cartesian to polar coordinates and from polar to cylindrical coordinates and so on (Hoffmann 1982). However, the point that Einstein wanted to make appears to have been more subtle (Stachel 1980). This is revealed by a remark added to a footnote. Einstein pointed out that "the independent variables $x_{v}$ " must be attributed to "the same numerical values of $x_{v}^{\prime}$ " (Einstein 1914b, 178; fn.; my emphasis). This amounts to the same maneuver introduced in the Besso memo: the new solution $g_{\mu \nu}^{\prime}$, obtained by switching to the coordinate system $x_{v}^{\prime}$, is evaluated with respect to the old coordinate system $x_{v}$, that is, $g_{\mu \nu}^{\prime}\left(x_{v}\right)$. It is important to emphasize that Einstein talked about the numerical values of variables. As we have seen, in Einstein's and most physicists' view at that time, the numbers $x_{v}$ do not simply serve to label points; the coordinate values $x_{v}$ are the points (see, e.g., Study $1914,82,91$ ). Thus the same numerical value $x_{v}$ with respect to the same coordinate system $K$ is the same point. Switching from a coordinate system to another is nothing but switching from a set of numbers $x_{v}$ to another set of numbers $x_{v}^{\prime}$ so that $x_{v}^{\prime}$ is some smooth function of $x_{v}$. In the generally covariant theory, all substitutions are allowed.

As we have seen, these numbers are meaningless. One needs to know $g_{\mu \nu}$ to be able to compute the relative distances of any point $x_{v}$ within the hole with respect to each material point $x_{v}$ on the boundary of the hole. Letting $x_{v}$ outside of the hole

\footnotetext{
${ }^{20}$ Following footnote 14 one could say that, before solving the field equations, physicists are in possession of a map with the treasure's coordinates $x=45, y=85$, but do not know the map's legend. The peculiarity of Einstein's theory of gravitation is that the legend of the map (the metric tensor) is, so to speak, hidden in the treasure chest (as it is a solution of the field equations). The theory aims to find the values of the coefficients $E, F, G$ at $x, y$ in the open ocean (the treasure); however, knowing $E, F, G$ (the map legend) as functions of $x, y$ is the precondition for physically locating $x, y$ on the globe. This is the root of all sorts of confusions, if one thinks in terms of the map and not in terms of the intrinsic geometrical structure of the globe.

${ }^{21}$ The coordinate system, as well as the components of the matter tensor, have remained unchanged outside of $L$.
} 
remain unchanged, one can introduce a new coordinate system $x_{v}^{\prime}$ within the hole and thus a new corresponding set of $g_{\mu \nu}^{\prime}\left(x_{v}^{\prime}\right)$. One might also calculate the new numerical values of the coefficients $g_{\mu \nu}^{\prime}$ as functions of the old coordinate values $x_{\nu}$. If the field equations are generally covariant, this will be an equally admissible solution. Thus, the field equations seem to predict two different sets of $g_{\mu \nu}$ components at the same point $x_{v}$, that is, the point at the same relative distances from the material points $x_{v}$ on the border of the hole. ${ }^{22}$ In principle, one could apply the same reasoning to a non-generally covariant theory of gravitation, but the predictability issue would not emerge. In fact, Einstein did not raise the Besso-Einstein argument against Nordström's scalar theory of gravitation (Stachel 1987), which, at that time, he considered as a good alternative to the Entwurf Theory (Einstein and Fokker 1914).

When Einstein left Zurich for Berlin in March 1914, he had fully convinced himself that a generally covariant theory was not desirable in the first place, since it violates the "condition that the fundamental tensor $g_{\mu \nu}$ should be completely determined" by the gravitation equations (Einstein and Grossmann 1914b, 216). In a paper with Grossmann, submitted in May, Einstein derived the field equations from a variational principle (Einstein and Grossmann 1914b) and convinced himself that the form of the Lagrangian was uniquely fixed by energy and momentum conservation. Einstein's results were summarized in the first systematic review of the Entwurf Theory that was presented in October of 1914 before the Prussian Academy of Sciences (Einstein 1914a) in Berlin. In $\$ 12$ of this paper, Einstein reformulated more clearly the 'proof' of a 'necessary restriction' in the choice of coordinates. Given the matter distribution $T_{\mu \nu} \neq 0$ outside of an empty region $\Sigma$ within which $T_{\mu \nu}=0$, the field equations determine the quantities $g_{\mu \nu}$ as functions of $x_{v}$ relative to the coordinate system $K$. Even if $g_{\mu \nu}$ and their first partial derivatives $\partial g_{\mu \nu} / \partial x_{v}$ are given on the boundary of $\Sigma$, we can still change the coordinates inside the region and thus obtain a different solution. Einstein used the nonstandard notation $G(x)$ to indicate these functions. His argument runs as follows:

Let us introduce a new coordinate system $K^{\prime}$ which coincides with $K$ outside of $\Sigma$, but deviates from $K$ inside of $\Sigma$ such, however, that the $g_{\mu \nu}^{\prime}$ relative to $K^{\prime}$ as well as the $g_{\mu \nu}$ (including their derivatives) are everywhere continuous. The totality of the $g_{\mu \nu}^{\prime}$ is symbolically denoted by $G^{\prime}\left(x^{\prime}\right), G^{\prime}\left(x^{\prime}\right)$ and $G(x)$ describe the same gravitational field. When we replace the coordinates $x_{v}^{\prime}$ by the coordinates $x_{v}$ in the functions $g_{\mu \nu}^{\prime}$, i.e., when we form $G^{\prime}(x)$, then this $G^{\prime}(x)$ also represents a gravitational field relative to $K$, which however, is not the same field as the factual (that is, the originally given) gravitational field. If

\footnotetext{
${ }^{22} \mathrm{~A}$ simplified version of the argument in terms of geographical maps might be the following. We want to calculate the values of the components $E(x, y), F(x, y), G(x, y)$ at the point $x=45, y=85$ in the middle of the ocean where no other marks are present (see footnote 14). Since the values of the components $E(45,85), F(45,85), G(45,85)$, depends on the projection, cartography seems to attribute two different sets of components $E, F, G$ and $E^{\prime}, F^{\prime}, G^{\prime}$ at the same point $x=45, y=85$. Thus, cartography cannot predict the values of $E, F, G$ univocally. The predictability of cartography can be saved once one realizes that the Cartesian coordinate numbers $x=45, y=85$ on the projection plane correspond to very different physical points on the globe depending on whether the calculations are made using the stereographic $E, F, G$ or the cylindrical $E^{\prime}, F^{\prime}, G^{\prime}$.
} 
we assume the differential equations of the gravitational field to be everywhere covariant, then they are satisfied for $G^{\prime}\left(x^{\prime}\right)$ relative to $K^{\prime}$ whenever they are satisfied for $G(x)$ relative to $K$. Therefore, they are also satisfied for $G^{\prime}(x)$ relative to $K$. There are then two different solutions $G(x)$ and $G^{\prime}(x)$ relative to $K$, even though the solutions coincide on the boundary of the domain $\Sigma$ (Einstein 1914a, 1067)

I have reported here this very well-known formulation of the argument since Einstein and his interlocutors repeatedly referred to 'the $\$ 12$-argument'. Thus, it is useful to have it at hand. The two-step scheme $G(x) \rightarrow G^{\prime}\left(x^{\prime}\right) \rightarrow G^{\prime}(x)$ described in this passage would indeed become from now on the standard formulation of Einstein's argument against general covariance. It shows that generally covariant field equations seem to attribute different values $G(x)$ and $G^{\prime}(x)$ of the gravitational potentials to the same $x_{v}$ within $\Sigma$ with respect to the same coordinate system $K$. As a matter of fact, if one considers the four numbers $x_{v}$ as being physically the same point of $K$, throughout a three-step calculation, the theory cannot predict the components of the gravitational potential at that point unequivocally. Thus, in Einstein's view, the argument provided a valid reason to introduce specialization in the choice of coordinates. Einstein imposed four non-covariant coordinate requirements written compactly as $B_{\mu}=0$ that guarantee the validity of the conservation laws (on which see Janssen and Renn 2015b, for more details). These relations were to hold in all those coordinate systems that Einstein called 'adapted' to a given gravitational field. In this way, Einstein was able to restrict the range of allowable $g_{\mu \nu}$ inside of $\Sigma$ and at the same time to leave it sufficiently large to comply with requirement of the equivalence principle (Abraham, 1914, 514).

\subsection{Embracing the mathematical overdetermination of the field equations}

The Entwurf Theory started to spark considerable interest. Lorentz insinuated that Einstein's use of 'adapted' coordinates was nothing but the introduction of privileged coordinate systems from the backdoor (Lorentz to Einstein, Jan. 1, 2015; CPAE, Vol. 8, Doc. 43). ${ }^{23}$ Nevertheless, he derived the Entwurf field equations from Hamilton's principle (Lorentz 1915). Moreover, he instructed his student Droste to calculate the gravitational field of a single mass point (Droste 1914) and two spherical fixed centers from the Entwurf equations (Droste 1915). After his Wolfskehl lectures his

\footnotetext{
${ }^{23}$ Einstein tried to convince Lorentz that this was not the case (Einstein to Lorentz, Jan. 23, 2015; CPAE, Vol. 8, Doc. 47). The situation, he claimed, was similar to the theory of surfaces. If one says that the line element is only allowed to take the form $d s^{2}=d p^{2}+d q^{2}$, then one will indeed say that the surface is flat. However, if one imposes the restriction $d s^{2}=E(p, q) d p^{2}+G(p, q) d q^{2}$, then one will say nothing about the nature of the surface. $F=0$ only imposes the choice of orthogonal coordinates. Spherical polar coordinates on a sphere and Cartesian coordinates on a plane are both orthogonal. Thus, Einstein did not think in terms of the intrinsic geometry of the surface, but in terms of the 'good' coordinate system in which the field equations are valid. The requirement $F=0$ does not fix the coordinate system uniquely as Cartesian inertial coordinates in pre-general-relativistic theories. However, the fact that the restriction $F=$ 0 is imposed once and for all is another instance of Einstein's confusion between 'coordinate restrictions' and modern 'coordinate conditions' discussed in footnote 17. This confusion will persist for at least two years after the final version of general relativity; see Sections 6.1 and 6.2.
} 
Wolfskehl lectures (CPAE, Vol. 6, appendix B) in Göttingen, which started to attract David Hilbert's interest in the theory, Einstein exchanged a few letters with the Göttingen physicist Paul Hertz and tried to convince him that his argument against general covariance was analogous to the case of two surfaces that are 'developable' into each other and thus geometrically identical (see Howard and Norton 1993). However, Einstein still considered Hertz's requirement " 'that the world be able to be developable upon itself'" atrocious (Einstein to Hertz, Aug. 14, 1915; CPAE, Vol. 8, Doc. 108). ${ }^{24}$ A few months later, his confidence in the Entwurf Theory started to deteriorate (CPAE, Vol. 8, Doc. 123), (CPAE, Vol. 8, Doc. 129). Einstein was lost again in the "chaos of possibilities" and started to reexamine the problem from the ground up (Einstein to Hertz, Aug. 22, 1915; CPAE, Vol. 8, Doc. 111).

On November 11, 1915, Einstein returned to a set of generally covariant vacuum field equations that he had introduced in 1913 (Einstein 1915c):

$$
R_{\mu \nu}=\kappa T_{\mu \nu},
$$

where $R_{\mu \nu}$ is what we now call the Ricci tensor. He replaced the restriction imposed by the conservation laws with the requirement that the determinant of the metric satisfied the condition $\sqrt{-g}=1$ (Einstein 1915c). Covariance with respect to transformations that preserve this condition is sufficiently broad to include transformations between inertial and accelerated coordinate systems. However, $g$ is a constant only if the scalar (the sum of the diagonal terms). ${ }^{25}$ of the energy tensor of matter $T_{\mu \nu}$ vanishes, $\Sigma T_{\nu}^{\mu}=T=0$, as in the case of the electromagnetic stressenergy tensor (Einstein 1915d). This way, Einstein arrived at field equations that are equivalent to generally covariant ones but specialized by the requirement $\sqrt{-g}=1$. If the determinant $g$ of the metric tensor is -1 , these field equations (with the Ricci tensor as a gravitational tensor) can be written in terms of the Christoffel symbols $\Gamma_{\mu \nu}^{\tau}$, which are now interpreted as the components of the gravitational field. For the vacuum field equations,

$$
\sum_{a} \frac{\partial \Gamma_{\mu v}^{\alpha}}{\partial x_{\alpha}}+\sum_{\alpha \beta} \Gamma_{\mu \beta}^{\alpha} \Gamma_{v a}^{\beta}=0,
$$

where the determinant of the metric is

$$
\left|g_{\mu \nu}\right|=-1 \text {. }
$$

On November 18, Einstein reported to the Prussian Academy of Sciences an approximate static spherically symmetric solution to these truncated field equations, which

\footnotetext{
${ }^{24}$ This shows, again, that Einstein did not think in terms of the intrinsic geometry of the surface, but in terms of the relations between meaningless coordinate numbers and real distances, that is, in terms of distortions performed by different map projections. Using different map projections, one can obviously calculate the values of $E^{\prime}, F^{\prime}, G^{\prime}$ and $E, F, G$ as functions of the same numbers $x=45, y=85$; however, $x=45, y=85$ is not the same physical point on the globe in both cases. What Hertz tried in vain to explain to Einstein was that all these 'maps' represent the same intrinsic properties of the surface. All intrinsic information-like which are the great circles, where they intersect, and so forth-is the same in all mappings, and it is preserved when the surface (or part of it) is bent without stretching.

${ }^{25}$ This is what we would now call the 'trace'.
} 
explained the anomalous advance of the perihelion of Mercury and the slight rotation of its elliptic orbit in addition to its elliptic motion. Before proceeding to solve the equations, he introduced the following remarks:

A point mass, the sun, is located at the origin of the coordinate system. The gravitational field this point mass produces can be calculated from these equations by means of successive approximations. Nevertheless, we should consider that the $g_{\mu \nu}$ are still not completely determined mathematically by the Eqs. 8 and 9 , because these equations are covariant with respect to arbitrary transformations of determinant 1 . Yet we are justified in assuming that all these solutions can be reduced to one another by such transformations that they are distinguished (by the given boundary conditions) formally but not, however, physically, from one another. Consequently, I am satisfied for the time being with deriving here a solution, without discussing the question whether the solution might be unique (Einstein 1915a, 832)

The case described is identical to that of the Besso memo, and indeed Einstein could rely on the calculations he had made with Besso (Janssen 2007). However, Einstein was now ready to accept that the field equations do not fix the solutions $g_{\mu \nu}$ uniquely, but only up to a coordinate transformation that respects the condition that the determinant of the metric is 1 . All such intertransformable solutions are only formally but not physically different. A genuine underdetermination would arise only if the solution remained underdetermined with respect to a definite coordinate system. However, Einstein must have now realized that this was not the case. For the given initial conditions, the worldlines of free-falling particles are geodesics of the $g_{\mu \nu}$. Since both the field equations and the equations of motion are generally covariant, if one switches to another coordinate system, the particle will cover the same path with respect to the new coordinate system.

Einstein's calculations revealed that the theory was able to account for the residual advance of $43^{\prime \prime}$ of arc per century that was unexplained in the Newtonian theory. This was a path-breaking result and Fokker reported that Einstein suffered heart palpitations following the discovery (Pais 1982, 253). A few days later, on November 25, 1915, Einstein (1915b) arrived at the field equations that today we can read in textbooks (albeit in slightly different notation). Imposing the condition $\sqrt{-g}=1$ again, Einstein could write the equations in terms of the Christoffel symbols and showed how they satisfied the conservation laws. Einstein's "boldest dreams have now been fulfilled" (Einstein to Besso, Dec. 10, 1915; CPAE, Vol. 8, Doc. 162). On December 14, he wrote to Schlick his "newly found result": an empirically sound theory, "whose equations are covariant with arbitrary transformations in the spacetime-variables" (Einstein to Schlick, Dec. 14, 1915; CPAE, Vol. 8, Doc. 165). As a consequence, space and time are now deprived of the "last vestiges [Rest] of physical reality [Realität]" (Einstein to Schlick, Dec. 14, 1915; CPAE, Vol. 8, Doc. 165; my emphasis).

The fact that the theory's equations could be simplified a posteriori by selecting and imposing the condition $\sqrt{-g}=1$ was deemed "of no epistemological significance" (Einstein to Schlick, Dec. 14, 1915; CPAE, Vol. 8, Doc. 165). Somehow crowning Einstein's triumph, on December 22, Karl Schwarzschild sent Einstein a 
letter from the eastern front communicating that he had already managed to find an exact solution for a 'mass point' (Einstein to Schwarzschild, Dec. 22, 1915; CPAE, Vol. 8, Doc. 169). Schwarzschild kept the condition $\sqrt{-g}=1$. After introducing the most general line element in rectangular coordinates, he went over to polar coordinates $\varphi, \vartheta, r$, which however do not satisfy the condition $\sqrt{-g}=1$ (for even in flat spacetime in spherical coordinates, $g=-r^{4} \sin ^{2} \vartheta$ ). He found a trick to circumvent this problem by defining a new radial coordinate $x=r^{3} / 3$ and a new angular coordinate $\varphi=-\cos \vartheta$. He then obtained a new set of variables, 'polar coordinates with the determinant 1', that might be called the Schwarzschild coordinate system. ${ }^{26}$ Presenting his solutions in these coordinates, he found that the equations for planetary orbits remain exactly the ones that Einstein had obtained in first-order approximation.

\section{The return of the point-coincidence argument in einstein's private correspondence}

\subsection{The einstein-ehrenfest correspondence}

Some of Einstein's closest interlocutors, such as Ehrenfest, were puzzled: "The day before yesterday," he wrote to Lorentz, "I received from Einstein the offprints of his latest papers on the theory of gravitation" (Ehrenfest to Lorentz, Dec. 23, 1915; SCHAL, Vol. 2, Doc. 247). Ehrenfest had always found it hard to make sense of Einstein's 'adapted coordinates' (see above Section 3.1). "But if I have properly understood what Einstein now intends, then his theory of November 25, 1915, claims to be entirely free of 'adaption'-restrictions of the coordinates. Is that so?!" (Ehrenfest to Lorentz, Dec. 23, 1905; SCHAL, Vol. 2, Doc. 247). In particular, Ehrenfest noticed that "[i]n 1914 Einstein developed an almost philosophical proof for the necessity of the 'adaptedness' of the coordinate system (I mean, e.g., §12 of his paper of 19.XI.1914.) Is this proof correct?" (Ehrenfest to Lorentz, Dec. 23, 1905; SCHAL, Vol. 2, Doc. 247). Einstein must have sensed that he owed an explanation to his correspondents (Ehrenfest to Lorentz, Dec. 23, 1905; SCHAL, Vol. 2, Doc. 247). In a letter to Ehrenfest, he described himself jokingly as the guy who each year "retracts what he wrote in the preceding year" (Einstein to Ehrenfest, Dec. 26, 1915; CPAE, Vol. 8, Doc. 173). Einstein was in a somewhat embarrassing situation of having to justify yet another retraction. Possibly at around that time, Einstein may have found an apt 'rhetorical device' to escape from his own argument against general covariance. ${ }^{27}$ This argument, the point-coincidence argument, made its first explicit appearance in a letter to Ehrenfest. This letter is well known in Einstein's scholarship:

\footnotetext{
${ }^{26}$ This nomenclature is not the usual one since what we call Schwarzschild were actually introduced by Droste; see Section 6.1.

${ }^{27}$ One can speculate that a paper by Kretschmann (1915; see Howard and Norton 1993) that was published on December 21 or discussions with Schlick (Engler and Renn 2013) might have suggested to Einstein a suitable 'turn of phrase'. As far as I can see, it has never been noticed that Einstein used the same turn of phrase as early as 1911; see Section 2.1 .
} 
In $\S 12$ of my paper of last year, everything is correct (in the first 3 paragraphs) [...] A contradiction to the uniqueness of the event does not follow at all from the fact that both systems $G(x)$ and $G^{\prime}(x)$, related to the same frame of reference, satisfy the conditions of the grav. field. [...]

1. the reference system has no real meaning

2. that the (simultaneous) materialization of two different $g$ systems (more aptly put, two different grav. fields) within the same area of the continuum is $[\ldots]$ impossible.

In place of $\$ 12$ the following consideration must appear. Whatever is physically real in events in the universe (as opposed to that which is dependent on the choice of a reference system) consist in spatio-temporal coincidences* For ex., the intersection points of two worldlines are real, or the statement that they do not intersect each other. [...] When two systems in the $g_{\mu \nu}$ 's (or gen., the variables used to describe the world) constituted in such a way that the second can be obtained from the first by mere spacetime transformation, then they are entirely equivalent. This is because they have in common all the spatialtemporal point coincidences, that is, all the observables (Einstein to Ehrenfest, Dec. 26, 1915; CPAE, Vol. 8, Doc. 173; my emphasis)

$*$ and in nothing else!

The apparent nonuniqueness of generally covariant equations would be objectionable only if more than one $g_{\mu \nu}$ system would be possible in the same coordinate system $K$. However, this is in principle impossible. Not only do the $g_{\mu \nu}$ constitute a physical field with a respect to a coordinate system, but also they tell us which coordinate system we are using. Therefore, the theory cannot predict the $g_{\mu \nu}$ at a point identified by its four coordinate numbers $x$. Changing coordinates, one obtains not only a new set of $G^{\prime}\left(x^{\prime}\right)$, but also a different physical meaning of the same quadruple of numbers $x=x^{\prime}$. Two worldlines that intersect at $x=x^{\prime}$ of $K$ according to $G(x)$ will indeed not intersect at the same point $x$ of $K$ according to $G^{\prime}(x)$. However, Einstein came to realize that this not a problem: the same point should be defined physically as where the same worldlines meet. Coordinate numbers $x$ serve only as a bookkeeping device for such coincidences. Thus, any choice of coordinate numbers is just good as any other (Einstein to Ehrenfest, Dec. 26, 1915; CPAE, Vol. 8, Doc. 173). Ultimately, according to Einstein, the point-coincidence argument showed "how natural the requirement of general covariance is" (Einstein to Ehrenfest, Dec. 26, 1915; CPAE, Vol. 8, Doc. 173). The specialization of $\sqrt{-g}=1$, Einstein insisted, does not change the essence of the matter.

Thus, Einstein was convinced that he had sufficiently refuted his own " philosophical' consideration" (Einstein to Ehrenfest, Dec. 29, 1915; CPAE, Vol. 8, Doc. 174). He wrote to Lorentz that he was "conducting a discussion with Ehrenfest" (Einstein to Lorentz, Jan. 1, 1916; CPAE, Vol. 8, Doc. 177), who was rather skeptical. As a matter of fact, Ehrenfest confessed to Einstein that Lorentz was not convinced either that his field equations with the condition $\sqrt{-g}=1$ were sufficient to determine das Geschehen, the events (Ehrenfest to Einstein, Jan. 1, 1916; CPAE, Vol. 8[12], Doc. 177a). However, Lorentz, differently from Ehrenfest, did not know about Einstein's letter with the point-coincidence argument, yet. Nevertheless, Ehrenfest, even 
after having discussed Einstein's counter-\$12-argument with his wife Tatjana and with Fokker, was still not convinced: "I will defend the philosophy of $\$ 12$ against your refutation" (Ehrenfest to Einstein, Jan. 1, 1916; CPAE, Vol. 8[12], Doc. 177a). After having repeated nearly literally some of the lines from Einstein's letter, he tried to explain his point of view with a thought experiment, concerning the curvature of light rays passing in the vicinity of the Sun. It was sort of an astronomical version of Young's double-slit experiment.

To make the rather abstract matter intuitive, Ehrenfest drew a picture of infinitely weak (i.e., of negligible self-gravitational energy) light waves coming from a star passing through the empty space around the Sun and reaching two telescope apertures that combine light waves to form a fringe pattern on a photographic plate (Fig. 2). The only "object of observation" is the "blackening or not blackening of a photographic plate behind both telescopes" (Ehrenfest to Einstein, Jan. 1, 1916; CPAE, Vol. 8[13], Doc. 177a). The physical situation is parametrized by the coordinate system $x_{1}, \ldots, x_{4}$, and fields $T_{\mu \nu}, g_{\mu \nu}$. Let us assume that the field equations predict a destructive interference on part of the plate: "Bravo the photrogr. plate is darkened (a true 'coincidence (explanation') claim" (Ehrenfest to Einstein, Jan. 1, 1916; CPAE, Vol. 8[13], Doc. 177a). However, one can proceed further and "see the philosophy of $\S 12$ at work," that in Ehrenfest's notation $G(x) \rightarrow G^{\prime}\left(x^{\prime}\right) \rightarrow G^{\prime}(x)$ :

In the cross-hatched region occupied by matter, we kept fix the description of the world-happening, whereas in the non-cross-hatched empty space (where there is only $\infty$ weak light!) the latter is strongly changed You look at me laughing quietly and you say 'go ahead, young friend, and describe if you like, the empty space

\section{with old coordinates $x$}

and brand new $G^{\prime}(x)$

nothing observable, no 'coincidence' would change!? Now I'm astonished and angry over your laugh and

\section{I claim with a clenched fist :}

'If with the old $x$ and the old $G(x)$ and the new $G^{\prime}(x)$ one calculates the darkening of the plate, then one should calculate the non-darkening of the plate

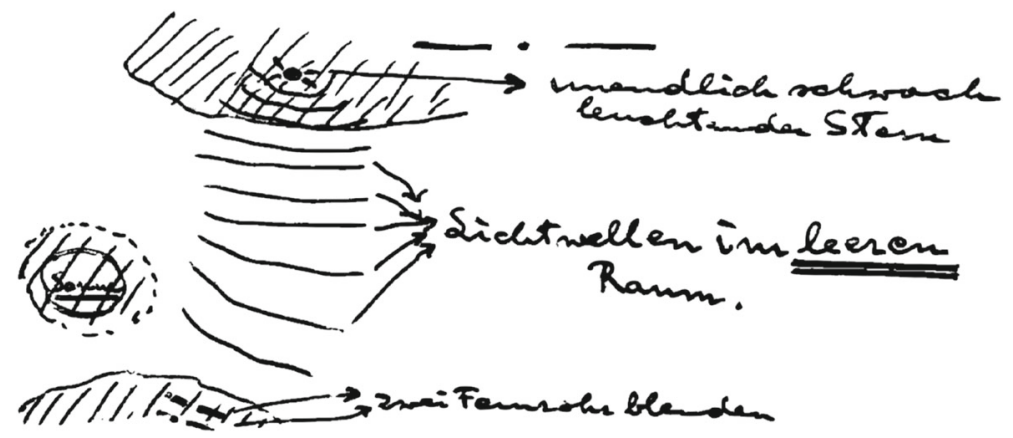

Fig. 2 Diagram in Ehrenfest's hand (Ehrenfest to Einstein, Jan. 1, 1916; CPAE, Vol. 8[12], Doc. 177a) 
with old $x$ and new $G^{\prime}(x)^{\prime}$ [The star, the sun and the telescope and the plate are not in empty but in matter space] (Ehrenfest to Einstein, Jan. 1, 1916; CPAE, Vol. 8[13], Doc. 177a).

The position of the star, the telescope aperture, and the plate in the matter-filled regions are fixed in a provisionally chosen coordinate system $K$ with $x$. One calculates a solution to the field equations and obtained $G(x)$; once one knows such coefficients, one can extract from the coordinate numbers $x$ the reciprocal distances of the Sun, the stars, the aperture, and the photographic plates and, in general, of any points $x$ with respect to $K$. Subsequently, the coordinates in the matter-free region are changed to the coordinate system $K^{\prime}$ so that $x^{\prime}=f(x)$. Following the instructions of Einstein's $\S 12$, one can produce a series of different solutions $G(x), G^{\prime}(x)$, $G^{\prime \prime}(x)$, and so forth on the same coordinate system $x$ : "Symbols are used precisely in the sense of your $\S 12$ !- Thus $G^{\prime}(x) \neq G(x)$ " (Ehrenfest to Einstein, Jan. 1, 1916; CPAE, Vol. 8[13], Doc. 177a). The trajectories of light rays appear to pass through different values of the same coordinate system $x$ in each case. "Do you concede this point or do you dispute it?", Ehrenfest asked Einstein rhetorically (Ehrenfest to Einstein, Jan. 1, 1916; CPAE, Vol. 8[13], Doc. 177a). If Einstein accepts Ehrenfest's reconstruction, then he has to admit that if the positions of the Sun, stars, aperture, and photographic plates are given and the theory cannot predict the interference patterns on the plate, which are precisely the 'coincidences' Einstein was talking about. Einstein should concede "with an icy polite smile" the failure of his "solemn formula [Beschwörungsformel]" in his Mercury-perihelion paper, that is, the claim that intertransformable solutions are formally different, but physically identical (Ehrenfest to Einstein, Jan. 1, 1916; CPAE, Vol. 8[13], Doc. 177a). ${ }^{28}$

However, by that time, Einstein had become fully confident that the Lochbetrachtung was flawed (Einstein to Besso, Jan. 3, 1916; CPAE, Vol. 8, Doc. 174). As he explained to Besso, the meeting of coordinate lines of $K$ is not real; real are only the "encounters [Begegnungen] of worldlines. In other terms: "nothing more" that pointcoincidences (Einstein to Besso, Jan. 3, 1916; CPAE, Vol. 8, Doc. 174). This was the root of Ehrenfest's difficulties as well. Ehrenfest "instinctively treat[ed] the reference system as something 'real'" (Einstein to Ehrenfest, Jan. 5, 1916; CPAE, Vol. 8, Doc. 180). Einstein considered this prejudice fully natural: "I myself needed so long to arrive at total clarity on this point" (Einstein to Ehrenfest, Jan. 5, 1916; CPAE, Vol. 8, Doc. 180). In order to convince Ehrenfest, Einstein invited him to depict a simplified version of his thought experiment (a star, an aperture, and a plate) on a tracing paper superimposed onto a flat letter paper. On the flat tracing paper, one can trace a regular graph in which coordinates directly represent actual lengths (the time coordinate is not taken into account). A coordinate transformation, which introduces curvilinear coordinates and a new set of $g_{\mu \nu}$, is represented as a deformation of the tracing paper. The boundary conditions at infinity, the coordinates of the star, the material point at the aperture, and the plate remain unchanged. The result is summarized in an often-quoted passage:

\footnotetext{
${ }^{28}$ The reference is to the passage cited above in Section 3.2.
} 
In the above special case you obtain all the solutions that are a consequence of general covariance in the following way. Trace [the star, the aperture and the plate] onto completely deformable tracing paper. Then deform the tracing paper arbitrarily in the plane of the paper. Then make another tracing on the letter paper [...] When you relate the figure once again to the orthogonal writing paper coordinates, the solution is mathematically different from the original, and naturally also with respect to the $g_{\mu \nu}$. But physically it is exactly the same, since the writing paper coordinate system is only something imaginary. Always the same points are illuminated on the plate [...] The essential thing is: as long as the drawing paper, i.e. 'space', has no reality, then there is no difference whatever between the two figures. It all depends on coincidences, e.g., whether the plate points are hit by the light or not. Thus, the distinction between your solutions $A$ and $B$ is merely a difference in presentation [Darstellung] with physical concordance [Übereinstimmung] (Einstein to Ehrenfest, Jan. 5, 1916; CPAE, Vol. 8, Doc. 180; my emphasis)

In pre-general-relativistic theories, we know in advance that the orthogonal tracing paper is flat and that rectangular coordinates (flat tracing paper) are a 'good' coordinate system, which is physically realizable in the form of a material scaffolding. The goal of physics was ultimately to correctly predict the paths of particles and light rays with respect to this coordinate system. We could, of course, use a different curvilinear coordinate system (the distorted tracing paper). Free particles would still follow timelike geodesics, and light rays would still follow null geodesics. However, they would impress different paths onto the letter paper. Nevertheless, before general relativity, we knew how to go back to the 'good' regular graph coordinate system, with respect to which the 'real' path of particles and light rays is defined. Since the deformed tracing paper was regarded as a 'bad' coordinate system, the theory's predictability was safe. By contrast, by abolishing the distinction between 'good' and 'bad' coordinate systems, at first sight, general relativity seems to be incapable of determining uniquely the paths of light rays with respect to the writing paper coordinate system. The illusion disappears once one realizes that the regular graph paper has no independent physical reality. Thus, there is no reason to complain that the same diagram's lines do not intersect at the 'same point' of the latter paper. The 'same point' is there where the same diagram worldlines intersect. ${ }^{29}$

\subsection{The Lorentz-Ehrenfest correspondence}

Einstein's and Ehrenfest's conceptual difficulties might surprise the modern reader who is already accustomed to considering coordinates as essentially arbitrary sys-

\footnotetext{
${ }^{29}$ The deformation of the tracing paper described by Einstein is equivalent to the introduction of different maps on the same projection plane $x, y$, which corresponds to the letter paper. On the map, the Greenwich meridian intersects the equator (as both are 'great circles', the largest circle that can be drawn on any given sphere) at different points $x, y$ and at different angles in different mappings, in stereographic, cylindrical, or other projections. However, this is not a problem for cartographers since they define the 'same point' as where the Greenwich meridian intersects the equator. This information is the same in all mappings.
} 
tems of parameters. However, these difficulties were clearly not idiosyncratic. Even a physicist of the status of Lorentz struggled to overcome the very same 'epistemological obstacle' (Kox 1985). On January 9, 1915, just a few days after Einstein's answer to Ehrenfest (Einstein to Ehrenfest, Jan. 5, 1916; CPAE, Vol. 8, Doc. 180), Lorentz sent a long letter expressing his concerns regarding general relativity and presenting his own argument against general covariance. Lorentz considered the truncated vacuum field equations, Eq. 8. He argued that, given one solution (I), $g_{\mu \nu}=F\left(x_{\alpha}\right)$, one can obtain a new one (II), $g_{\mu \nu}^{\prime}=F^{\prime}\left(x_{\alpha}^{\prime}\right)$, where $g_{\mu \nu}^{\prime}=g_{\mu \nu}+\delta_{\mu \nu}$. This is done by introducing a small change of the spatial coordinates $x_{1}, x_{2}, x_{3}$ in the form $x_{v}^{\prime}=x_{v}+\zeta_{v}$ (where $\zeta_{v}$ is a small quantity of the 'first' order), whereas $x_{4}^{\prime}=x_{4}$. He imposed a condition on $\zeta_{v}$ that lets $|g|=-1$ remain unchanged. The result was the following:

If, e.g., I have the solution $g_{\mu \nu}=F\left(x_{\alpha}\right)$ (symbolically expressed), and replace $x_{\alpha}$ by $x_{\alpha}^{\prime}$, then by the transformation formulas, I can provide the values of $g_{\mu \nu}^{\prime}$. I can express them in $x_{\alpha}^{\prime}$; suppose $g_{\mu \nu}^{\prime}=F^{\prime}\left(x_{\alpha}^{\prime}\right)$. Then $g_{\mu v}=F^{\prime}\left(x_{\alpha}\right)$ will also satisfy equations (8). This is a new solution, differing from the first. [...] (I) and (II) now, in fact, differ physically, since in field (I) a material point moves uniformly along a straight line, whereas one can easily see that this is not the same in field (II). [...] One can also, taking (II) as a starting point [...] [and] derive a third solution; one can continue in this way [...] From the above it follows, it seems to me, that in the case we are considering of the matter-free field, equations (8) are, with continuity and the conditions at infinity, insufficient to determine the field; in contrast with Laplace's equation $\Delta \varphi=0$, which in connection with the additional conditions requires that $\varphi=0$ (Lorentz to Ehrenfest, Jan. 9, 1916; SCHAL, Vol. 2, Doc. 230).

Lorentz insisted that one would find a similar overdetermination by considering the Sun's gravitational field calculated by Einstein. The coordinate transformation $x_{v}^{\prime}=$ $x_{v}+\zeta_{v}$ can be applied to it as well. One can also make the case that $\zeta_{v}$ differs from 0 also in the space occupied by the Sun, so then field (II) will pertain to a 'changed' Sun. However, one can also set $\zeta_{v}=0$ within the Sun and change only the external field so that the same distribution of matter would produce different fields. To escape this overdetermination, one could try to introduce additional conditions (e.g., that the field is Minkowskian at infinity, symmetric, etc.). However, in Lorentz's view, this would not limit our freedom of choosing $\zeta_{v}$ in the general case.

Ehrenfest replied immediately on the very same day (Ehrenfest to Lorentz, Jan. 9, 1916; SCHAL, Vol. 2, Doc. 230), with a letter written in German and a brief introduction and conclusion written in Dutch, "under the pressure of orthographicalgrammatical boundary conditions" (Ehrenfest to Lorentz, Jan. 9, 1916; SCHAL, Vol. 2, Doc. 230). The day before, he had just received Einstein's answer to his question about the curvature of light beams (Einstein to Ehrenfest, Jan. 5, 1916; CPAE, Vol. 8, Doc. 180). Ehrenfest enclosed Einstein's letter within the letter he sent to Lorentz. Ehrenfest did not find Einstein's reply very clear; however, thanks to a long discussion with his wife, he came to realize that Einstein was "entirely right" (Ehrenfest 
to Lorentz, Jan. 9, 1916; SCHAL, Vol. 2, Doc. 230). Ehrenfest relied again on the example of the curvature of light beams, which he meant to be "Ganz im Sinn des $\S 12$ ” (Ehrenfest to Lorentz, Jan. 9, 1916; SCHAL, Vol. 2, Doc. 230).

He considered a material space made by a star, the Sun, an aperture, and a photographic plate on Earth (Fig. 3). Exactly like in Einstein's §12, in the matter-free region around this material system, two observers $A$ and $B$ introduced two tensor fields $g_{\mu \nu}^{A}(x)$ and $g_{\mu \nu}^{B}(x)$ on the same coordinate system $x_{1}, x_{2}, x_{3}, x_{4}$, whereas the $g_{\mu \nu}^{0}(x)$ field in the matter region remained the same. When light rays coming from a star enter into the matter-free region, they go through different coordinate values $x_{1}, x_{2}, x_{3}, x_{4}$ according to $g_{\mu \nu}^{A}(x)$ and $g_{\mu \nu}^{B}(x)$. In fact, the path of light rays is nothing but the shortest chain of $d s$ whose links are null space-time length so that small increments $d x$ have natural spacetime distance $d s^{2}=0$. Which small increments $d x$ correspond to such a natural distance depends on the $g_{\mu \nu}$ systems, $g_{\mu \nu}^{A}(x)$ or $g_{\mu \nu}^{B}(x)$, that one uses to make the calculations. Thus, ultimately, given the coordinate system $x$, light rays will go through different values of $x$, since the same coordinate differences $d x$ correspond to different real distances $d s$. However, in the meantime, Einstein had managed to convince Ehrenfest that the paths of light rays, calculated according to $g_{\mu \nu}^{A}(x)$ and $g_{\mu \nu}^{B}(x)$, will meet again at the same point $x_{1}, x_{2}, x_{3}, x_{4}$ on the matter region in which the coordinate system has remained unchanged and that they will do so with the same angle in both cases. Thus, one obtains the same darkening of the photographic plate.

Ehrenfest realized a key point. Many conceptual confusions emerged because physicists were not thinking 'geometrically' in terms of the intrinsic structure of

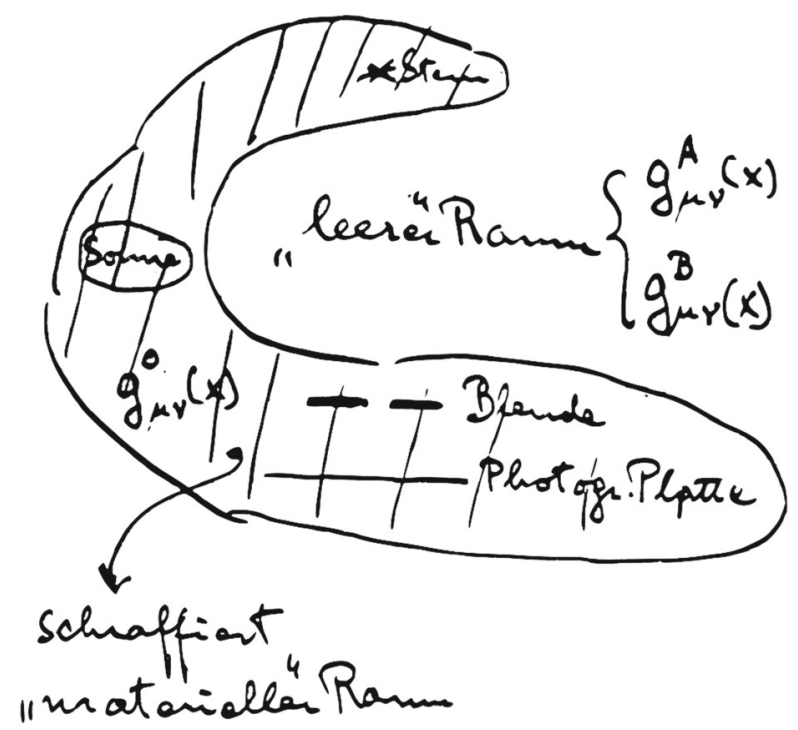

Fig. 3 Diagram in Ehrenfest's hand (Ehrenfest to Lorentz, Jan. 9, 1916; SCHAL, Vol. 2, Doc. 230) 
spacetime, but rather algebraically in terms of transformations of variables. ${ }^{30}$ Thus, he suggested to represent the 'world' as a four-dimensional $E$ surface embedded in a five-dimensional $Z$ space. The transformation considered by Einstein was nothing but bending without stretching of the parts of the surface between the stars and the aperture. The remaining parts of the surface were kept unchanged. This bending produced a different surface: "This is, geometrically speaking, the result of $\S 12$ " (Ehrenfest to Lorentz, Jan. 9, 1916; SCHAL, Vol. 2, Doc. 230). The two surfaces look differently in the embedding space (like a plane, a cone, and a cylinder look differently). However, since they are developable onto each other, they share all intrinsic geometrical properties (like a plane, a cone, and a cylinder have the same flat geometry). The distance between two points, measured along the surface (i.e., along the shortest line joining them), is unchanged, and so is the angle between two lines that meet at a point. The paths of light rays are nothing but some of such lines. Thus, although observers $A$ and $B$ bend the central part of the surface differently in the matter-free region, inasmuch as that this does not stretch the surface, the light rays will meet again at the same angle with the telescope.

Ehrenfest was eager to know whether Lorentz considered his two solutions (I) and (II) as equivalent in this sense. Lorentz replied immediately; he "had read only a part of [Einstein's letter] [Einstein to Ehrenfest, Jan. 5, 1916; CPAE, Vol. 8, Doc. 180"] and he had realized that "he was entirely right". "I wrote to him straight away to withdraw my objections of yesterday," he concluded (Lorentz to Ehrenfest, Jan. 10, 1916; SCHAL, Vol. 2, Doc. 231). Lorentz described to Ehrenfest with great clarity the 'epistemological obstacle' he had found difficult to overcome:

In connection with this I now realize that in the two fields I and II that I spoke of in my last letter [Lorentz to Ehrenfest, Jan. 9, 1916; SCHAL, Vol. 2, Doc. 230], it is true that the separate phenomena do not take the same course in relation to the coordinate system, but that in both of them the coincidences do occur in the same way [...] I was too much a prisoner of the idea that our equations must fully reproduce [...] the relations between the phenomena and the chosen coordinate system, whereas we can be happy if they duly reproduce the mutual relations between the phenomena. In physics, one has up to the present (though it appears to be unnecessary) aimed at drawing up equations that fully determine the phenomena in relation to a chosen coordinate system [...] But it now turns out that the 'field equations' are of a different nature. We cannot claim that they determine the $g_{\mu \nu}$. Instead, we must say that when for the $g_{\mu \nu}$ we take arbitrary functions that satisfy the equations, and then calculate all phenomena with these $g_{\mu \nu}$, we shall reproduce all coincidences well (Lorentz to Ehrenfest, Jan. 11, 1916; SCHAL, Vol. 2, Doc. 231; my emphasis).

For practical purposes, one should perhaps restrict the choice of $g_{\mu \nu}$ through suitable additional conditions. This restriction "would, however, now have no sort of deeper meaning and could be brought about in an arbitrary way" (Lorentz to Ehrenfest, Jan.

\footnotetext{
${ }^{30}$ In other terms, they reasoned in terms of different map distortions and not of the unique shape of the globe.
} 
11, 1916; SCHAL, Vol. 2, Doc. 231; my emphasis). Thus, Lorentz realized that also the condition $\sqrt{-g}=1$ has nothing special. We chose "the coordinate system such that $g=-1$," only for the reason that this would give us "the simplest possible solution (i.e., the values of $g_{\mu \nu}$ ) of the field equations" (Lorentz to Ehrenfest, Jan. 11, 1916; SCHAL, Vol. 2, Doc. 231). However, a less simple solution would be just as legitimate. "As far as the reproducing of the coincidences is concerned, the choice of the coordinate system must be entirely indifferent" (Lorentz to Ehrenfest, Jan. 11, 1916; SCHAL, Vol. 2, Doc. 231). Worldlines do not take the same course in relation to the coordinate system, but in both of them, coincidences do occur in the same way. Indeed, at closer inspection, a worldline is not a succession of coordinate values, but a succession of (possible) coincidences. "Theoretical physics, "Lorentz concluded, "now begins to resemble the geometria situs" (Lorentz to Ehrenfest, Jan. 11, 1916; SCHAL, Vol. 2, Doc. 231).

Lorentz "congratulated Einstein on his brilliant results" (Lorentz to Ehrenfest, Jan. 11, 1916; SCHAL, Vol. 2, Doc. 231; see also Lorentz to Ehrenfest, Jan. 12, 1916; SCHAL, Vol. 2, Doc. 232). However, as Ehrenfest pointed out in his reply, Lorentz's comparison of relativity theory to the geometria situs was too hasty (Ehrenfest to Lorentz, Jan. 12, 1916; SCHAL, Vol. 2, Doc. 233). "I know very well that you mean only to indicate a direction, not a point: the development from a lesser to a greater “deformability"' (Ehrenfest to Lorentz, Jan. 12, 1916; SCHAL, Vol. 2, Doc. 233). According to Ehrenfest, general relativity was indeed "more 'topological' than ordinary theories, yet still not fully topological" (Ehrenfest to Lorentz, Jan. 12, 1916; SCHAL, Vol. 2, Doc. 233). The two solutions of the field equations that can be 'deformed' into one another in the sense of $\S 12$, that is, through a mere coordinate transformation, are equivalent (Ehrenfest to Lorentz, Jan. 12, 1916; SCHAL, Vol. 2, Doc. 233). This sort of deformation "causes the Einsteinian theory to resemble analysis situs, it is true, but to remain very certainly infinitely more limited" (Ehrenfest to Lorentz, Jan. 12, 1916; SCHAL, Vol. 2, Doc. 233). The correct analogy, according to Ehrenfest, was with Gauss's theory of surfaces. The four-dimensional world cannot be arbitrarily deformed like the rubber band of the geometria situs; it can only be "deformed "without being stretched" (Ehrenfest to Lorentz, Jan. 12, 1916; SCHAL, Vol. 2, Doc. 233) like how a cylinder can be developed onto a flat surface. This was precisely what Einstein stubbornly refused to accept from Hertz in August 1915: the world is developable onto itself (as discussed in Section 2.2). Einstein thought of $E, F, G$ as a calculational device that, given $d q$ and $d p$, spits out $d s$, and at the same time $E, F, G$ are fields with certain values at $p, q$. However, this way, he seems to have missed the key point: the entire class of inter-transformable $E, F, G$ represents the same curved surface, with the same Gaussian curvature.

Einstein agreed with the "truly Ehrenfestian description of the telescope affair" (Einstein to Ehrenfest, Jan. 17, 1916; CPAE, Vol. 8, Doc. 182). In general, he was pleased with the reception that the theory had within the Leiden community, "a brilliant nook on this barren planet" (Einstein to Ehrenfest, Jan. 17, 1916; CPAE, Vol. 8, Doc. 182). Einstein wrote to Lorentz a few days later: "I see that you have thought over the theory entirely and have familiarized yourself with the idea that all of our experiences in physics refer to coincidences" (Einstein to Lorentz, Jan. 17, 1916; CPAE, Vol. 8, Doc. 245). This point of view quite consequently requires 
the formulation of generally covariant equations. Einstein read the Ehrenfest-Lorentz correspondence and confirmed that he himself "had hit upon this erroneous stance that corresponds to the standpoint held by you in the first of your letters" (Einstein to Lorentz, Jan. 17, 1916; CPAE, Vol. 8, Doc. 245). Einstein conceded that although he had presented the field equations in the $\sqrt{-g}=1$ coordinates, it was important to present the field equations in a generally covariant form to avoid any arbitrariness. However, he also insisted that it was necessary to specialize the coordinate systems in some natural way, although he did not find a proper way to do so (Einstein to Lorentz, Jan. 17, 1916; CPAE, Vol. 8, Doc. 245). Einstein was particularly fond of how Lorentz had presented the point-coincidence-general covariance relations and he asked him to make these "considerations available to other physicists as well" (Einstein to Lorentz, Jan. 17, 1916; CPAE, Vol. 8, Doc. 245).

Lorentz agreed that a systematic presentation of the theory was a worthy endeavor (Lorentz to Ehrenfest, Jan. 22, 1916; SCHAL, Vol. 2, Doc. 235). As he suggested to Ehrenfest, he "could talk on this subject on Mondays at 10 o'clock for a few weeks, and use these lectures as the basis for a short paper or a little book" (Lorentz to Ehrenfest, Jan. 22, 1916; SCHAL, Vol. 2, Doc. 235). Indeed, it was Lorentz who used for the first time the point-coincidence argument in a public lecture in February 1916, explicitly referring to a correspondence that he had with Einstein (Lorentz, 1917b, 1342; fn. 3). Lorentz suggested that we could investigate the gravitational field of the Sun and we could perform a sort of 'scattering' experiment in a finite region surrounded by detectors. Given certain initial conditions, we would send a great number of material points and light rays, moving in all directions and with different velocities, and note all coincidences recorded by the detectors, that is, all "data by astronomical observations" that one could imagine to be infinitely detailed (Lorentz 1917b, 1343).

One can represent these coincidences in a four-dimensional diagram, as a 'field figure', a maze of worldlines and of light rays and their intersections, which can ultimately be everywhere dense, a sort of continuously distributed matter without internal forces. This way, "each observed coincidence could be represented by an intersection of worldlines" (Lorentz 1917b, 1343). The claim that Mars $(M)$ is at a certain point at a certain instant with respect to the Sun $(Z)$ means that an astronomical sight lines up the planet, a fixed star $(S)$ and the telescope on $A$. This implies that the trajectory of a light ray joining the star and the orbit of the planet, passing through a telescope comoving with Earth $(A)$, and leaving marks on a photographic plate is interrupted by the passage of Venus $(V)$ when the handles of a clock are on a certain position on the dial (Fig. 4). In previous theories, there was only one correct field figure; in general relativity, any deformation that allowed all intersections, and thus all coincidences and all observational data, to remain unchanged is just as good as any other (field-figure-I and field-figure II in Fig. 4). ${ }^{31}$ Mars (M) is at the same point at the same time in all cases.

\footnotetext{
${ }^{31}$ Once again, Lorentz seems to conceive a field figure as a map projection on a flat projection plane (see footnote 12, above). A change of the coordinate system corresponds to a different map projection and thus to a deformation of the field figure as in Fig. 4.
} 


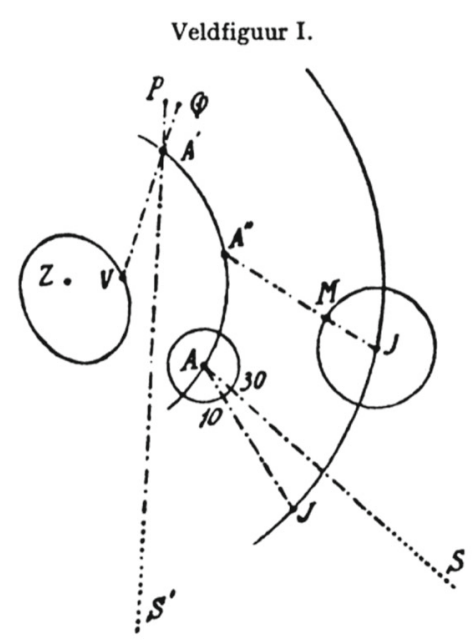

FIG. 1.

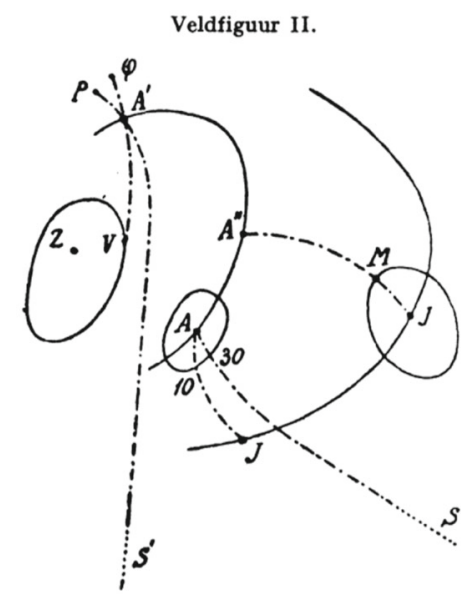

FIG. 2.

Fig. 4 Two equivalent field figures (Lorentz 1917a)

\section{The point-coincidence argument in Einstein's 1916 paper: getting rid of the last remnant of materiality of the coordinate system}

As most readers might recall, Einstein used the point-coincidence argument for the first time in a published writing in his 1916 review paper (Einstein 1916a; see Sauer 2005; Janssen 2005; Gutfreund and Renn 2015), which was submitted in March. "I have worked very hard on a final formulation of general relativity that has now been fully developed" (Einstein to Zangger, Mar. 1, 1916; CPAE, Vol. 8[12], Doc. 196a). The argument appears in one of the first sections of the paper at the conclusion of what might appear as a sort of autobiographical, 'rational' reconstruction of the evolution of Einstein's attitude toward coordinates. "In classical mechanics, as well as in the special theory of relativity, the coordinates of space and time have a direct physical meaning" (Einstein 1916a, 773). To say that a point event has coordinate $x_{1}$ means measuring off with a unit rod $x_{1}$ times from the origin of the coordinate system $K$ along the axis of $x_{1}$. To say that an event has coordinate $x_{4}$ means that a unit clock at rest in $K$ measured off $x_{4}$ periods at the occurrence of the event starting from $x_{0}$. Coordinates are labels that tell us which spacetime points we are referring to and at the same time they tell us where those points are, their relative spacetime distances from other points with respect to a 'good' coordinate system $K$. "This view of space and time has always been in the minds of physicists, even if, as a rule, they have been unconscious of it" (Einstein 1916a, 774).

Working within the framework of special relativity, Einstein realized that this conception of coordinates could not be extended to accelerated systems. A coordinate system $K^{\prime}\left(x^{\prime}, y^{\prime}, z^{\prime}, t^{\prime}\right)$ accelerating relative to a 'good' system $K(x, y, z, t)$ can be in principle maintained in a Born rigid motion, allowing for a direct measurement of 
$x, y, z$. However, one has to abandon the direct measurement of $t$ with a clock (Einstein 1916g). A coordinate system $K^{\prime}\left(x^{\prime}, y^{\prime}, z^{\prime}, t^{\prime}\right)$ rotating around the $z=z^{\prime}$-axis with respect to $K$, on the contrary, defies even this criterion of rigidity and does not allow for the measurement of $x, y, z$, by means of rigid rods (Stachel 1989). Since Einstein considered a uniformly rotating frame $K^{\prime}$ as equivalent to a system at rest $K$ in a static gravitational field, he had to abandon the comfortable setting in which coordinate numbers meant also distances. One might ask what the physical content of a theory is in which the positions of material points and the values of field quantities are expressed as functions of the coordinates, but the latter are not measurable. In an enormously famous but quite elliptic passage, which does not betray the conceptual struggle needed to achieve the result, Einstein reminded his readers of what is actually observable in a physical theory:

That this requirement of general covariance, which takes away from space and time the last remnant of physical materiality [Gegenständlichkeit], is a natural one, will be seen from the following reflexion. All our spacetime assessments [Konstatierungen] invariably amount to a determination of spacetime coincidences. If, for example, events consisted merely in the motion of material points, then ultimately nothing would be observable but the meetings [Begegnungen] of two or more of these points. Moreover, the results of our measurings are nothing but assessments [Konstatierungen] of such meetings of the material points of our measuring instruments with other material points, coincidences between the hands of a clock and points on the clock dial, and observed point-events happening at the same place at the same time.

The introduction of a system of reference serves no other purpose than to facilitate the description of the totality of such coincidences (Einstein 1916a, 771; translation modified).

One might read this passage as saying that the result of any measurement should ultimately ascertain whether a mark on one scale (a movable pointer or such) coincides with a certain mark on another scale. This statement does not entail any reference to coordinates. In a given coordinate system, two events are coincident if their coordinates are the same, but the relationships between space-time coincidences are independent of the coordinate system used (Ohanian 1976, 253). Thus, coordinate numbers serve as individual 'names' for such coincidences (as they tell which coincidence is referred to) and do not carry any further 'metrical' information (as they do not tell us where this coincidence is).

The relationships between coordinate numbers and real distances can be extracted from the formula for the line element, which is now written so that the summation over repeated indices is assumed:

$$
d s^{2}=g_{\mu \nu} d x_{\mu} d x_{\nu} .
$$

This formula tells us that when $d s$ and $d x_{1}, d x_{2}, d x_{3}, d x_{4}$ are known numbers, the expression of the line element represents one equation for the determination of the unknowns $g_{\mu \nu}$. On the contrary, if $g_{\mu \nu}$ and $d x_{1}, d x_{2}, d x_{3}, d x_{4}$ are known, the equation spits out the number $d s$. The circular way in which these two operations are 
connected is at the origin of Einstein's difficulties of illustrating the relation between the predicted values of $g_{\mu \nu}$ and the observed ones, the ones that are measured with rods and clocks. This is revealed by the surprisingly misleading way in which Einstein presented the latter relations in the review paper.

After an introduction to the absolute differential calculus (Part $B$ ), Einstein derived the gravitational field equations (Part $C$ ) from a Lagrangian $\delta \int \mathrm{H} d \tau=0$, and by imposing the condition $\sqrt{-g}=1$. He then obtained the field equations in the following form:

$$
\begin{gathered}
\frac{\partial}{\partial x_{\alpha}} \Gamma_{\mu \nu}^{\alpha}+\Gamma_{\mu \beta}^{\alpha} \Gamma_{\nu \alpha}^{\beta}=-\kappa\left(T_{\mu \nu}-\frac{1}{2} g_{\mu \nu} T\right), \\
\sqrt{-g}=1 .
\end{gathered}
$$

After a discussion of the form that $T_{\mu \nu}$ assumes in particular cases, in the last section, Einstein discussed the Newtonian approximation of weak fields, Minkowski's flat boundary conditions, and the slow motion of particles. He applied this approximating procedure to obtain an approximate solution to his field equations for the exterior field of a static, spherically symmetric mass. In the first Newtonian approximation, Einstein obtained the following values for $g_{\mu \nu}$, written in arbitrary Cartesian coordinates, so that $r=\sqrt{x_{1}^{2}+x_{2}^{2}+x_{3}^{2}}$ is the radial distance between the Sun and a certain point. In this coordinate system, the approximate solution of the field equations takes the following form:

$$
\begin{aligned}
& g_{\rho \sigma}=-\delta_{\rho \sigma}-\alpha \frac{x_{\rho} x_{\sigma}}{r^{3}}(\rho, \sigma=1,2,3), \\
& g_{\rho 4}=g_{4 \rho}=0, \\
& g_{44}=1-\frac{\alpha}{r},
\end{aligned}
$$

where $\alpha=\kappa \mathrm{M} / 4 \pi$, with $M$ being the mass of the Sun. It can be shown that this solution satisfies the condition $\sqrt{-g}=1$. What remains now is to show how these predicted values can be compared with experience. Einstein's approach was somehow puzzling. As we have seen, it can be assumed that, in a small region of space (over a single space-like hypersurface), we can introduce a Cartesian coordinate system in which $g_{11}=-1, g_{22}=-1$, and $g_{33}=-1$, so that coordinates are measurable by unit rigid rods that have the same length in every position and every orientation. In the presence of the gravitational field, however, this construction would fail over a large region of space. Even in first approximation, the coefficients $g_{11}, g_{22}$, and $g_{33}$ are not constant, as Einstein had initially believed, but just like the coefficient $g_{44}$, functions of the coordinates. If we want to check whether the calculated $g_{\mu \nu}$ values are 'true', we will need to measure the observed $g_{\mu \nu}$ values using rods and clocks. Einstein described an idealized measurement procedure as follows. A unit rod laid along the radius $x_{1}$ satisfies

$$
d s^{2}=-1, \quad d x_{2}=d x_{3}=d x_{4}=0,
$$

but does not measure directly the coordinate $x_{1}$,

$$
-1=g_{11} d x_{1}^{2}, \quad g_{11}=-\left(1+\frac{\alpha}{r}\right) .
$$


On the contrary, a rod laid in the perpendicular direction reads directly the coordinates since it satisfies

$$
\begin{gathered}
d s^{2}=-1, \quad d x_{1}=d x_{3}=d x_{4}=0, \\
-1=g_{22} d x_{2}^{2}, \quad g_{22}=1 .
\end{gathered}
$$

A clock placed at rest in the gravitational field satisfies

$$
d s=1 d x_{1}=d x_{2}=d x_{3}=0,
$$

and measures

$$
1=g_{44} d x_{4}^{2}, \quad g_{44}=-\left(1+\frac{\alpha}{r}\right) .
$$

It is indeed unsettling that, in Einstein's view a rod measured in coordinates is shortened by the gravitational field by a factor of $\sqrt{g_{11}}$ and a clock is slowed down by a factor of $\sqrt{g_{44}}$ as if equal differences in coordinates $x_{1}$ and $x_{4}$ had a physical meaning, denoting equal distances from the origin. This approach is visible in Einstein's 'backward derivation' of the red shift, in which equal differences in time coordinates give unequal proper time differences $d x_{4}=1 / \sqrt{g}_{44}$ (Earman and Glymour 1980b). A similar issue emerges, which follows Einstein's derivation of light deflection (Earman and Glymour 1980a). Einstein introduced the velocity of light $\gamma=1$ "in the sense of Euclidean geometry" (Einstein 1916a, 806), and then he calculated the distorted values:

$$
\gamma=\sqrt{\left(-\frac{g_{44}}{g_{22}}\right)}=1-\frac{a}{2 r}\left(1+\frac{x_{2}^{2}}{r^{2}}\right) .
$$

Thus, again, light rays follow paths in which small differences $d x_{v}$ of the successive values of $x_{v}$ are always $d s=0$ in Cartesian coordinates. These paths are distorted by the gravitational field $g_{\mu \nu}$. However, this way, $\gamma$ would depend on the value of $g_{44}$ and $g_{22}$.

In the approximation procedure adopted by Einstein, in which one starts from Cartesian coordinates, it seems natural to interpret coordinate differences as distances (e.g., $r$ as the distance from the Sun) and $g_{\mu \nu}$ as a field that distorts such measurements. However, this approach has an obvious drawback. Since the components of $g_{\mu \nu}$ are defined only up to a coordinate transformation, with a different choice of coordinates, rods and clocks would be shortened and slowed down differently by different factors $g_{\mu \nu}$. However, at this point, Einstein must have been aware that the relationships between coordinates and rods and clocks measurements are actually reversed, as he more aptly wrote a few lines later. Instead of measuring in terms of coordinates and considering the rod distorted, one should take "one and the same rod, independently of its place and orientation, as a realization of the same interval $[-d s]$ " (Einstein 1916a, 820; my emphasis). As it turned out, "Euclidean geometry does not hold even to a first approximation in the gravitational field" (Einstein 1916a, 820). That is, near the Sun, it is impossible to cover a sufficiently large region of space with a coordinate system in which $g_{11}=g_{22}=g_{33}=-1$, and $d s=-1$ in every position and every orientation. This is like trying to wrap a flat piece of paper around a sphere. If we assume that identical clocks (say, identical atoms) always measure the same $d s$ without being influenced by the gravitational field, in general, it is 
impossible to choose a coordinate system in which time coordinates are the result of clock measurements, that is, where $g_{44}=1$. Similarly, identical rods and identical clocks, wherever they are placed in the gravitational field, always measure the same velocity of light $c$.

Einstein's presentation of the practice of rods and clocks measurement in general relativity in terms of a field that distorts rods and clocks, and thus the paths of particles and light rays, reveals the fact that he seemed to vacillate between two different mental models: (a) a practically useful mental model in which $g_{\mu \nu}$ extract different 'natural' differences $d s$ from equal differences in coordinates and (b) a theoretically more adequate mental model in which equal natural distances $d s$ correspond to different coordinate distances according to different $g_{\mu \nu}$ systems. Since Einstein did not think in geometrical terms (the intrinsic curvature of spacetime) but saw (10) as a calculation tool to translate coordinate distances into real distances and vice versa, he seemed to switch from one mental model to the other depending on the circumstances. More precisely, although Einstein knew that (b) is, 'in theory', the correct approach, he often reasoned 'in practice' in terms of (a). Given the coordinate differences of two points, what is the distance between them? The line element (10) gives different answers to this question depending on the components of the $g_{\mu \nu}$. In my view, this way of posing the question is the source of most of the difficulties that we have encountered so far.

It is of course not that, using different intertransformable $g_{\mu v}$ as conversion factors, we calculate different $d s$ from the same coordinate increments as in (a); rather, as in (b), using different $g_{\mu \nu}$, we can recover the same $d s$ from different coordinate increments. ${ }^{32}$ In spacetime, worldlines are given by an infinitely large number of subsequent values of $x_{1}, x_{2}, x_{3}, x_{4}$. Free-falling particles in an empty region of spacetime occupy the series of $x_{1}, x_{2}, x_{3}, x_{4}$, which satisfies the constraint that the chain of time-like $d s$ connecting two successive coordinate increments $x_{v}$ and $x_{v}+d x_{v}$ is the longest among all alternatives. If one remains attached to (a), then the same series of coordinate numbers $x_{1}, x_{2}, x_{3}, x_{4}$ that represent the longest time-like worldline in the $g_{\mu \nu}$ system will not in $g_{\mu \nu}^{\prime}$. Thus, the worldlines of two colliding free-falling particles $A$ and $B$ that intersect at the worldpoint $x_{1}, x_{2}, x_{3}, x_{4}$ according to $g_{\mu \nu}$, in general, will not according to $g_{\mu \nu}^{\prime}$. However, according to (b), free-falling particles follow the longest chain of $d s=1$ in all $g_{\mu \nu}$-systems; the fact that such world-lines can be parametrized by different series of $x_{1}, x_{2}, x_{3}, x_{4}$ is meaningless. In all $g_{\mu \nu}$ systems, the same worldlines of $A$ and $B$ intersect at the same point, because the same point is defined operationally as where the same worldlines intersects. "Every physical description resolves itself into a number of statements, each of which refers to the spacetime coincidence of two events $A$ and $B$ " (Einstein 1916f, 65). Coordinates

\footnotetext{
${ }^{32}$ Darrigol (2015) labels (a) 'perverted geodesy' and (b) 'concrete geodesy'. In our cartographic analogy, model (a) corresponds to taking the Cartesian coordinate differences $d x, d y$ on the projection plane as meaningful as in footnote 12 and distances in different maps as distortions; (b) means that every map represents the same latitude and longitude on the curved globe and that the projection distortions are meaningless as in footnote 11. According to Valente (2018), Einstein embraced the perverted geodesy point of view. However, to me, this claim seems to be too strong and in conflict with too many passages in which Einstein declared coordinates as meaningless.
} 
$x_{1}, x_{2}, x_{3}, x_{4}$ do not tell us where such coincidences happen with respect to a given coordinate system; the four numbers $x_{1}, x_{2}, x_{3}, x_{4}$ are only a bookkeeping system to keep track of which coincidences we are referring to in a given coordinate system.

The convincing power of the point-coincidence argument ultimately depended probably on the fact that it could present what was a radical redefinition of what is observable in physics as if, in a more careful analysis, it were already implicit in previous physics practices. This is probably a rational reconstruction of how Einstein himself stumbled on the point-coincidence argument. As we have seen, as early as 1911 (see Section 2.1), Einstein had pointed out that, in physics and in everyday experience alike, "the position of an object in space is based on the specification of the point on a rigid body with which that event or object coincides" Einstein 1916f, 4; my emphasis). Einstein used this very same parlance again in his popular book on relativity (Einstein 1916f) that he started to plan at the beginning of 1916 (Einstein to Besso, Jan. 3, 1916; CPAE, Vol. 8, Doc. 178) and concluded by the end of the year. As he pointed out further in the booklet, one needed only to take this conception to its extreme consequences.

Physicists used to think that the goal of a physical theory was to predict the successive positions $x, y, z$, of, say, a material point with respect to a suitably chosen reference frame at time $t$ measured by clocks placed at fixed positions on such scaffolding. At closer inspection, however, what they were actually able to predict was only the "the encounters [Begegnungen] of this [material] point with particular points" of a particular physical system that we have chosen as a "reference-body", at the moment of "the encounter of the hands of clocks with particular points on the dials" (Einstein 1916f, 65). Thus, ultimately, even in pre-general-relativistic theories, spacetime predictions always referred to the encounters of at least two physical systems.

Pre-general-relativistic physics considered one system as somehow 'more important' than the other, i.e., the system that could be treated as an external, nonaccelerating system unaffected by the interactions being described. One wants to know where the material point is on the scaffolding and not the other way around. Thus, it was natural to think of the scaffolding as a three-dimensional rigid reference body (endowed with clocks) which can be isolated from the phenomena under investigation. Where a material point is could be expressed unambiguously in terms of relative distances from the origin. However, because of the 'equivalence principle', there is no way to identify non-accelerating reference bodies external to the gravitational system. But, using accelerating frames of reference in a relativistic framework is riddled with difficulties. Already, in the case of uniform acceleration, the rigid reference body had to be substituted with a three-dimensional deformable reference body in Born rigid motion. Since this reference body could not be put into rotation, Einstein had to capitulate and drop the very notion of a reference scaffolding. He finally realized that physics could still get by with a more tenuous material medium, a "reference-mollusk" (Einstein 1916f, 67), that is a non-rigid reference body-say, a cloud of particles filling the space, each carrying a clock. Physics can make meaningful predictions about the encounters of such particles: such and such free-falling clock collides with such and such particle, as the hand of the clock coincides with 
a certain mark on the dial. This redefinition of what is observable in physics, however, implied a more subtle conceptual reorientation. The very question of where this encounter happens has become meaningless. "The last remnant of physical materiality [Gegenständlichkeit]" of the coordinate system, Einstein could triumphantly claim, has been dissolved (Einstein 1916a, 776).

\section{The (very) last remnant of materiality of the coordinate system}

\subsection{A Galilean coordinate system: the physical meaning of the requirement $\sqrt{-g}=1$}

In spite of his efforts to convince his readers of the viability of the requirements of general covariance, it is somewhat surprising that, in his 1916 paper, Einstein did not give the field equations in a generally covariant form but rather in the form Eq. 11, that is, by imposing the coordinate condition $\sqrt{-g}=1$. As it was suggested by a manuscript for an unpublished appendix to the review article (CPAE, Vol. 6, Doc. 31), Einstein considered it possible to derive the field equations from a variational principle with the Ricci scalar $R$ chosen as a Lagrangian without the $\sqrt{-g}=1$ condition. He also knew already that the conservation laws are satisfied without introducing such specialization. Thus, Einstein considered the field equations as generally covariant but only presented them in a special coordinate system in which the distinction between tensors and tensor densities disappears, simplifying the calculations. However, in May 1916, after sending to Ehrenfest the proofs of his review article (Einstein to Ehrenfest, Apr. 29, 1916; CPAE, Vol. 8, Doc. 218), Einstein wrote to him that his specialization of the coordinate system was "not just based on laziness," seemingly implying that the postulate $\sqrt{-g}=1$ was more than a calculation trick (Einstein to Ehrenfest, May 24, 1916; CPAE, Vol. 8, Doc. 218). Even if the field equations should be presented in a fully generally covariant form, Einstein, nevertheless, considered that it is desirable to find a physical motivation for specializing the coordinate system a posteriori.

Exacts solutions were of course of particular interest. Schwarzschild's papers delivering exact internal and external solutions were respectively issued in April (Schwarzschild 1916b) and May (Schwarzschild 1916a) in polar coordinates of determinant 1. Hans Reissner (1916) soon found a generalization of the Schwarzschild metric, including an electrical charge relying on Einstein's generally covariant formulation of Maxwell's equations (Einstein 1916b). Nevertheless, approximation schemes played an equally important role in the early application of the theory. At that time, Einstein, like Schwarzschild himself, was searching for wave solutions of field equations, which were expected in a field theory of gravitation by analogy to electrodynamics. Einstein initially failed to find such a solution in $\sqrt{-g}=1$ coordinates, raising doubts that it was even possible. Such coordinate systems were in fact in conflict with the coordinate condition given by Eq. 5 that enable writing down a wave equation (Einstein to Schwarzschild, Feb. 19, 1916; CPAE, Vol. 8, Doc. 220).

Einstein found a workaround in June, as one can infer from a letter to Lorentz: "I myself worked on the integration of the field equations in first-order approximation 
and examined gravitational waves. The results are in part astonishing. There are three kinds of waves, though only one type transports energy" (Einstein to Lorentz, Jun. 17, 1916; CPAE, Vol. 8, Doc. 226). The reason for the breakthrough was again a contribution of the work of the Leiden community. ${ }^{33}$ Lorentz's Monday morning lecture played an important role in making the Leiden physicists familiar with the formalism of general relativity (Kox 1992). In May, Droste (1917), Lorentz's doctoral student, found independently of Schwarzschild an exact spherical symmetric static solution for the mass point, however, dropping the condition $\sqrt{-g}=1$. In June, (De Sitter 1917) started to calculate the motion of planets and the Moon using an approximate solution of the field equations. De Sitter used yet another coordinate system that was also used by Lorentz in his lectures (Lorentz 1917c), a coordinate system in which the velocity of light depends on position but not on direction. ${ }^{34}$ De Sitter must have managed to convince Einstein that gravitational wave solutions are easier to find in such Lorentz coordinates than in Einstein coordinates. He communicated to him the approximate values of the diagonal elements of the gravitational field of a mass point in Lorentz coordinates in a private correspondence.

On June 22, Einstein presented to the Prussian Academy of Sciences a paper on gravitational waves (Einstein 1916d). He calculated the approximation

$$
g_{\mu \nu}=-\delta_{\mu \nu}+\gamma_{\mu \nu} \quad \delta_{\mu \nu}= \begin{cases}0 & \text { if } \mu \neq v, \\ 1 & \text { if } \mu=v .\end{cases}
$$

Einstein introduced the trace-reversed tensor $\bar{\gamma}_{\mu \nu}=\gamma_{\mu \nu}-1 / 2 \delta_{\mu \nu} \gamma$, which plays the role of a four-vector potential in electrodynamics. ${ }^{35}$ The specialization contained in Eq. 13 is conserved if one performs an infinitesimal transformation on the coordinates $x_{\mu}^{\prime}=x_{\mu}+\xi^{\mu}$. One can then impose the condition (5), ${ }^{36}$ which is a linearized approximation that allows rewriting the field equations for weak fields in a form familiar in electrodynamics:

$$
\sum_{\nu} \frac{\partial^{2}}{\partial x_{v}^{2}} \bar{\gamma}_{\mu \nu}=2 \kappa T_{\mu \nu}
$$

For a given material system with energy-momentum $T_{\mu \nu}$ at the origin of the coordinate system, $\bar{\gamma}_{\mu \nu}$ can then be determined "in a manner analogous to that of retarded potentials in electrodynamics" (Einstein 1916d, 688). As the simplest example of application, Einstein calculated the gravitational field of a mass point of mass $M$, resting at the point of origin of the coordinates, and obtained the diagonal values of the $g_{\mu \nu}$ : "Mr. De Sitter sent me these values by letter; they differ from those which I previously gave [in Eq. 12] only in the choice of the system of reference" (Einstein 1916d, 692). Einstein further calculated the components of a gravitational

\footnotetext{
${ }^{33}$ For the first extensive analysis of this episode, see Kennefick (2007, 53ff.). More technical details are discussed by Weinstein (2015, sec. 3.1).

${ }^{34}$ This coordinate system is therefore called 'isotropic'. I will refer to it as the system of Lorentz coordinates.

${ }^{35}$ See footnote 19.

${ }^{36}$ This is the harmonic coordinate condition, which corresponds to the electromagnetic Lorenz gauge.
} 
field stress-energy pseudotensor $t_{v}^{\mu}$ in this coordinate system. De Sitter's coordinate choice did not satisfy the condition $\sqrt{-g}=1$; however, Einstein realized that this choice of coordinates was advantageous in performing calculations. Indeed, in the rest of the paper, Einstein derived solutions of Eq. 14 representing plane gravitational waves (whose wavefronts are flat, rather than curved). Einstein classified the waves into three types: longitudinal, transverse, and a new type of wave. Using the component $t_{4}^{4}$ of the stress-energy pseudotensor that he calculated in the first part of the paper, he determined the energy transported by such waves. ${ }^{37}$ Einstein realized that this component vanishes for longitudinal and transverse waves.

Einstein wrote the following to de Sitter on June 22, the day he submitted his paper on gravitational waves (Einstein 1916d) to the academy: "Your solution for the mass point [...] [o]bviously [...] differs from my old one in the choice of the coordinate system, but not intrinsically" (Einstein to de Sitter, Jun. 22, 1916; CPAE, Vol. 8, Doc. 227). However, Einstein realized that the two types of waves that do not transport energy in Lorentz coordinates could be transformed away using Einstein coordinates satisfying $\sqrt{-g}=1$. Thus, in an appendix to the paper, Einstein suggested that the latter have a "deep-seated physical justification" (Einstein 1916d, 696). Einstein's $\sqrt{-g}=1$ coordinate system $K$ should be regarded as a 'good' preferred coordinate system, whereas de Sitter's Lorentz coordinate system $K^{\prime}$ is suitable for calculations but 'bad' from a physical point of view. Einstein went so far as labeling these coordinate systems 'Galilean'. Indeed, to a certain extent, their role resembles that of an inertial system, a system with respect to which the real position of particles and components of fields are defined. As one can infer from a remark made on the margin of Einstein's letter, de Sitter was puzzled: "What is this 'Galileischer Raum'? ${ }^{38}$ Could one not just as well say the 'Aether'?” (de Sitter's margin note, in Einstein to de Sitter, Jun. 22, 1916; CPAE, Vol. 8, Doc. 227).

De Sitter returned to this issue in a letter written at the beginning of July. ${ }^{39} \mathrm{He}$ pointed out that "in order to determine the coordinate system one needs in general four conditions" (de Sitter to Einstein, Jul. 7, 1916; CPAE, Vol. 8[12], Doc. 227a). As Hilbert (1915) had explained, no definite solution of generally covariant field equations can be found unless these four additional noncovariant equations are imposed (e.g., those conditions given in Eq. 5). However, once a solution is obtained, one must be able to transform the solution freely to other coordinate systems. On the contrary, if the four conditions fixing the coordinate system are chosen once and for all, "this would mean that one relinquishes relativity" and falls back to an absolute space and time that exists "independently of any physical observation and from the entirety of physics" (de Sitter to Einstein, Jul. 7, 1916; CPAE, Vol. 8[12], Doc. 227a). Therefore, de Sitter found it unsuitable that Einstein in his letter "called the coordinate system characterized through $-g=1$ as a 'Galileischer Raum ${ }^{40}$," (de Sitter to Ein-

\footnotetext{
${ }^{37}$ The pseudotensor $t_{\mu \nu}$, unlike a tensor, can vanish at a point in one coordinate system but not in others.

${ }^{38}$ Galilean space. Quoted in German in the original Dutch text.

${ }^{39}$ In the manuscript, the letter is dated June 7. However, since it refers to Einstein's paper of June 26, the correct date is probably July 7.

${ }^{40}$ See footnote 38
} 
stein, Jul. 7, 1916; CPAE, Vol. 8[12], Doc. 227a). De Sitter pointed out that, imposing this requirement, one, like Schwarzschild, would be forced to exclude polar coordinates; however, there is nothing 'ungalileian' in the usual polar coordinates (de Sitter to Einstein, Jul. 7, 1916; CPAE, Vol. 8[12], Doc. 227a). As a matter of fact, Droste was able to find the same solution as Schwarzschild without imposing the condition $\sqrt{-g}=1$.

According to de Sitter, imposing four conditions on the coordinates is indeed "a kind of freezing or immobilization of the space, so that the choice of coordinates is independent of the matter which might be present in space or of its motion and energy changes" (de Sitter to Einstein, Jul. 7, 1916; CPAE, Vol. 8[12], Doc. 227a). In principle, one might also call this choice of coordinates a "frozen space in Galileo's name and deem the other spaces as non-real or apparent". However, in de Sitter's view, it must be emphasized that this distinction is "fully arbitrary" (de Sitter to Einstein, Jul. 7, 1916; CPAE, Vol. 8[12], Doc. 227a). "If other physicists had discovered in this or other spaces something advantageous, they would have the same right to call their space as the 'true' one and define it as 'galileian' or maybe even the 'aether'" (de Sitter to Einstein, Jul. 7, 1916; CPAE, Vol. 8[12], Doc. 227a). Hence, the role of the four coordinate conditions is to help obtain a solution of a particular kind of problem. For example, the coordinate condition (5) allows one to find especially simple solutions in the case of linearized gravitational equations in empty space but might not be suitable in other cases. Thus, once a solution is obtained, the coordinate condition may be discarded, as one may throw away the proverbial ladder after climbing it up.

De Sitter interpreted Einstein's preferences for $\sqrt{-g}=1$ coordinates in this way, but he was not sure whether he had misunderstood Einstein's stance. In his reply, written a week later, Einstein insisted on the importance "of further specializ[ing] the [coordinate] system in a natural way, even in the interests of better comparability of the found solutions" (Einstein to de Sitter, Jul. 15, 1916; CPAE, Vol. 8, Doc. 235). Indeed, without introducing a specialization of the coordinate system, no solution can be found. However, Einstein admitted that it was "not good if in my letter I called the system $\sqrt{-g}=1$ 'Galilean"' (Einstein to de Sitter, Jul. 15, 1916; CPAE, Vol. 8, Doc. 235). Only a coordinate system in which all $g_{\mu \nu}$ 's are constant should be called that. Einstein, in principle, agreed with de Sitter's interpretation of the distinction between 'real' and 'apparent'. If one finds a wave solution of the field equations, there are two possibilities. Either this wave-like process is independent of the choice of the coordinate system or it is just a coordinate effect. In the latter case, the wave motion can be transformed away by switching to another coordinate system and it is nonreal. De Sitter's $K^{\prime}$ coordinates, that is, Lorentz coordinates, were more advantageous for calculation. However, Einstein's $K$ satisfying $\sqrt{-g}=1$ had ultimately a special status from a physical point of view since it allows only waves that transport energy. Gravitational waves with respect to $K$ are 'real', whereas with respect to $K^{\prime}$ they are 'unreal' (Einstein to de Sitter, Jul. 15, 1916; CPAE, Vol. 8, Doc. 235).

However, Einstein's Leiden interlocutors were puzzled. As Lorentz wrote to de Sitter a day later, it was hard to avoid "the impression that E[instein] becomes somewhat unfaithful to his own doctrine now that he is going to privilege one particular 
kind of coordinate system and speak of a 'Galilei'schen Raum'" (Lorentz to de Sitter, Jul. 16, 1916). If the requirement $\sqrt{-g}=1$ is used exclusively, this seems to violate the spirit of general relativity. Even if Einstein insisted that the field equations were still valid in all coordinates (Einstein to De Donder, Jul. 23, 16; CPAE, Vol. 8, Doc. 240), the distinction between a set of 'good' coordinate systems with respect to which phenomena look real and 'bad' one with respect to which they are 'apparent' is introduced again from the backdoor. The attitude of the Leiden group toward this issue seemed to have been quite different. De Sitter wrote a little paper in English in July, in which he put the problem of the choice of coordinates and notion of coordinate condition in a way that is closer to the modern view (De Sitter 1916a).

De Sitter emphasized that the 10 differential equations $G_{\mu \nu}=\kappa T_{\mu \nu}$ do not determine $g_{\mu \nu}$ completely. If some set of $g_{\mu \nu}$ is a solution to Einstein's equations in one coordinate system $x_{1}, x_{2}, x_{3}, x_{4}$, it should also be a solution in any other coordinate system $x_{1}, x_{2}, x_{3}, x_{4}$. The coordinate system is specified by certain added coordinate conditions, four equations for $g_{\mu \nu}$ that must not be covariant. Different choices of these four conditions might suit different problems; however, choice among them is fully conventional. In de Sitter's words,

An important remark must still be made with respect to the equations $\left[G_{\mu \nu}=\right.$ $\left.\kappa T_{\mu \nu}\right]$. Since $\left[G_{\mu \nu}^{\prime}=G_{\nu \mu}^{\prime}\right]$ and $\left[T_{\mu \nu}=T_{\nu \mu}\right]$ there are 10 of these equations, and there are also coefficients $g_{i k}$ to be determined. However, the equations $\left[G_{\mu \nu}=\kappa T_{\mu \nu}\right]$ are not independent of each other, but four of them are consequences of the other six. They, therefore, are not sufficient to determine the $g_{\mu \nu}$. This is essential. For the $g_{\mu \nu}$ determine the character of the four-dimensional system of reference, and the principle of general relativity requires that this system can be arbitrarily chosen [...] In order to determine the $g_{\mu \nu}$ completely we must to the equations $\left[G_{\mu \nu}=\kappa T_{\mu \nu}\right.$ ] add four additional conditions, which can be arbitrarily chosen, and which determine the choice of the system of reference. According to the form of these additional conditions the $g_{\mu \nu}$ will be different functions of the coordinates, the equations of motion will be different, the course of rays of light will be different, but there will always be the same intersections of world-lines, and consequently all observable phenomena will be exactly the same: they will only be described by a different system of space coordinates and a different time. One system may be more convenient than another-this is a matter of taste; but we cannot say that one system is true and another false (De Sitter 1916b, 418-419). ${ }^{41}$

Thus, de Sitter resorted to the point-coincidence argument to show that the choice of the coordinate condition is arbitrary, and that no such condition, including Einstein's preferred condition $\sqrt{-g}=1$, is privileged. The observable phenomena, that is, the 'coincidences', are not affected by the coordinate system used, and this is all that counts from a physical point of view.

\footnotetext{
${ }^{41}$ Latin indeces has been substituted by Greek indeces.
} 
After writing two fundamental papers on the emission and absorption of radiation in quantum theory (Einstein 1916e, h), in the fall of 1916, Einstein stayed for a few weeks in the Netherlands. In the "unforgettable hours" (Einstein to Besso, Oct. 31, 2016; CPAE, Vol. 8, Doc. 270) he spent with Lorentz, Ehrenfest, Nordström, and younger physicists like Droste and Fokker, discussions regarding this issue must have inevitably ensued. Besides starting to address questions of a cosmological nature with de Sitter (de Sitter to Einstein, Nov. 1, 1916; CPAE, Vol. 8, Doc. 272), in November, Einstein sent to Ehrenfest the galley proofs of his paper in which he derived the field equations from a Lagrangian without imposing the coordinate condition $\sqrt{-g}=1$ and asked him to show them to 'co-conspirators' (X-Brüdern) in Leiden (de Sitter to Einstein, Nov. 1, 1916; CPAE, Vol. 8, Doc. 272). The paper was nothing but the March 1916 appendix (CPAE, Vol. 6, Doc. 31). Einstein was now satisfied that the Hamiltonian function did not become heavily complicated even if one lets the coordinate system be general, that is, without imposing the $\sqrt{-g}=1$ condition. In particular, Einstein insisted that the derivation of the conservation laws that he obtained before imposing the condition $\sqrt{-g}=1$ holds in all coordinate systems. As he wrote to Hermann Weyl, "one can see how the free choice [Wählbarkeit] of the reference system can be of advantage in the calculation" (Einstein to Weyl, Nov. 23, 1916; CPAE, Vol. 8, Doc. 278). Einstein's flirtation with the existence of privileged coordinate systems seems to have come to an end.

\subsection{The point-coincidence argument again: the correspondence with Gustav Mie}

At the beginning of December of 1916, Droste presented his spherically symmetric solution with additional results in the form of a doctoral dissertation in Dutch, which was published in the spring of 1917 (Droste 1917). Opening the dissertation, Droste insisted that "he has not made use of the coordinate system often used by Einstein, where $\sqrt{-g}=1$ " (Droste 1917, XII). According to Droste, Einstein's coordinate choice, on the one hand, did not significantly simplify the calculations and, on the other hand, diverted the attention from the particular characteristics of the equations to that of the coordinate system (Droste 1917, XII). The choice of the coordinate system was recognized as fully arbitrary, "although one choice will be more effective than the other" (Droste 1917, XII). As he wrote to Ehrenfest in the spring of 1917, Einstein found "Droste's dissertation [...] extraordinarily beautiful" (Einstein to Ehrenfest, May 25, 1917). Nevertheless, as late as the spring of 1917, he probably still believed that $\sqrt{-g}=1$ coordinates somehow reflected the objective properties of spacetime. The condition is, for example, used in Einstein's cosmological work (Einstein 1917a, 145). Einstein was again convinced to free himself of this last remnant of materiality of the coordinate system through an exchange with the Leiden community (for more details on which see Kennefick 2007, 63ff.; see also Weinstein 2015, 254ff.).

In June, Ehrenfest wrote to Einstein that Nordström, newly engaged, was "preparing to write you (Ehrenfest to Einstein, Jun. 14, 1917; CPAE, Vol. 8, Doc. 352). Nordström got back to Einstein only in August after his marriage ceremony (Nord- 
ström to Einstein, Oct. 23, 1917; CPAE, Vol. 8, Doc. 393). He had calculated the distribution of energy in a gravitational field with spherical symmetry, which is the quantity $t_{4}^{4}$ (for which see also Lorentz 1918, §48). Nordström relied on the notation introduced by Droste in his dissertation but used Schwarzschild's polar coordinates satisfying $\sqrt{-g}=1$. He rewrote this equation in Cartesian coordinates so that $\sqrt{-g}=1$ would be satisfied and calculated the components of the energy of the gravitational field using the formula of Einstein's 1916 general theory of relativity review paper (Einstein 1916b, 806, eq. 50). Einstein had not performed this calculation in that paper since he made his calculations using Lorentz coordinates. Nordström hence reached the unsettling conclusion that

$$
t_{4}^{4}=0 .
$$

In other terms, " $\mathrm{t}]$ he gravitational field, therefore, would have then no energy outside of the body" (Einstein to de Sitter, Sep. 22, 1917; CPAE, Vol. 5, Doc. 382), which was in disagreement with Einstein's result in his 1916 gravitational wave paper. Puzzled, Nordström checked his results and attached an addendum to the letter, in which he wondered whether "[t]he entire contradiction could lie in the fact that we are using different coordinate systems" (Einstein to de Sitter, Sep. 22, 1917; CPAE, Vol. 5, Doc. 382). In his 1916 gravitational wave paper, Einstein had used Lorentz coordinates "characterized by the velocity of light being equal in all directions" (Einstein to de Sitter, Sep. 22, 1917; CPAE, Vol. 5, Doc. 382). As Nordström pointed out, an exact solution for the point mass in this coordinate system was also investigated by Droste in his dissertation. Relying on Droste's calculations, but keeping them at the same level of approximation that one can find Einstein's work, he found

$$
t_{4}^{4}=\frac{1}{4} \frac{\alpha^{2}}{r^{4}}
$$

Thus, the energy density of the gravitational field does not vanish in Lorentz coordinates but does in Einstein's $\sqrt{-g}=1$ system. As a consequence, the coordinate system that Einstein believed to be physically 'privileged' has a rather unphysical implication that the gravitational field of a point mass has no energy.

Einstein must have suspected some computational mistake. However, in October, Nordström confirmed his calculations using the approximate values and again obtained $t_{4}^{4}=0$ (Nordström to Einstein, Oct. 23, 1917; CPAE, Vol. 8, Doc. 393) when the system of coordinates is chosen so that the condition $\sqrt{-g}=1$ is satisfied. Thus, Einstein's preferred coordinate system had the counterintuitive implication that there appears to be no energy in the gravitational field of a point mass (Nordström 1918a, b; see also Schrödinger 1918). Using a different system of coordinates, this might not be the case, as in Lorentz-de Sitter's coordinates. After discussing the matter with Fokker, Nordström came to the conclusion that there was no particular reason why it was useful to impose such a condition. Indeed, $\sqrt{-g}=1$ coordinates have a more direct physical interpretation, since they provide a fixed absolute measuring unit (Anderson and Finkelstein 1971). The unit volume of a four-dimensional ele- 
ment is the same in coordinate units as in natural units. ${ }^{42}$ However, it does not seem necessary for coordinate differences and natural distances to be expressed in the same units, and the latter might be treated as pure numbers (Nordström to Einstein, Oct. 23, 1917; CPAE, Vol. 8, Doc. 393).

At about the same time, the physicist Gustav Mie, who had critically followed the development of Einstein's theory since 1913, sent to Einstein at his request (Einstein to Mie, Dec. 14, 1917; CPAE, Vol. 8, Doc. 407) the published version of his Göttingen Wolfskehl lectures on relativity (Mie 1917a, b, c). On December 22, Einstein repaid the courtesy by sending Mie his 1916 'Hamiltonian' paper (Einstein 1916c) and his 1916 paper on gravitational waves (Einstein 1916d). He admitted, however, that the latter contained a computational error. By that time, Einstein must have realized that in that paper he had used the trace-reversed tensor $\bar{\gamma}_{\mu \nu}$ instead of $\gamma_{\mu \nu}$ to calculate the energy density of gravitational waves $t_{4}^{4}$ (Einstein to Mie, Dec. 22, 1917; CPAE, Vol. 8, Doc. 416). Commenting on Mie's lectures, Einstein pointed out that he disagreed with Mie's "considerations about the necessity of the existence of preferred coordinate systems". "They can easily cause confusion," he added (Einstein to Mie, Dec. 22, 1917; CPAE, Vol. 8, Doc. 416). He revealed to Mie that he was preparing a new paper on gravitational waves, which he submitted by the end of December. It was presented by Planck at the end of December (Einstein 1918c).

In this classic paper, besides providing the so-called quadrupole formula (Kennefick 2007, 65ff.), Einstein presented the role of coordinates and coordinate conditions in a way that appears closer to that of a modern textbook. Coordinate transformations involve four arbitrary functions, he wrote, so that four coordinate conditions must be added to the six independent equations to permit unique solutions (Einstein 1918c, 155). This applies to linearized gravity as well. Given a solution $g_{\mu \nu}=-\delta_{\mu \nu}+\gamma_{\mu \nu}$, one might obtain a different solution by an infinitesimal coordinate transformation $x_{v}+\xi_{v}$ leading to $g_{\mu \nu}^{\prime}=-\delta_{\mu \nu}+\gamma_{\mu \nu}^{\prime}$. However, this does not change the physical content of the solution. The freedom in the choice of the vector $\xi_{v}$ is merely an expression of the freedom of choosing coordinates. Gravitational waves that transport no energy can always be generated from a field-free system by a mere coordinate transformation (Einstein 1918c, 161). As a matter of fact, one can always introduce a sinusoidal behavior of $\gamma_{\mu \nu}$ by introducing the Minkowski metric in nonstandard coordinates. However, those waves are only apparent and should be distinguished from real waves, which cannot be introduced in this way.

Einstein's attitude toward the role of coordinates in physics at that time can be better appreciated by considering how he reacted to Mie's counterobjection (Mie to Einstein, Feb. 5, 1918; CPAE, Vol. 8, Doc. 456). To make his case, Mie introduced a paradox that he had used in his Göttingen lectures (Mie 1917a, b, c). He considered a rod at rest in a certain coordinate system. Using light rays as a standard, the rod appears to be straight. However, by introducing a fast-changing gravitational field and thus a writhing coordinate system, the rod would appear to be curved and to move

\footnotetext{
${ }^{42}$ Hence, the label 'unimodular' that we use for such coordinates, where 'modulus' is the volume element.
} 
like a snake, without any physical cause (Illy 1988). The two coordinate systems are indeed geometrically equivalent; however, physically, the first coordinate system is clearly better than the second one (Mie to Einstein, Feb. 5, 1918; CPAE, Vol. 8, Doc. 456). However, at this point, Einstein considered Mie's yearn for a 'good' coordinate system unacceptable. To make is stance clear, Einstein resorted again to the pointcoincidence argument to reinstate his conviction that coordinates in general relativity are meaningless:

I do not agree at all with your reflection about the bent (flapping) rod. All physical descriptions that yield the same observable relations (coincidences) are equivalent in principle, provided that both descriptions are also based on the same laws of nature. The choice of coordinates can have great practical importance from the point of view of clarity of description; in principle, though, it is entirely insignificant. It means nothing that 'arbitrary gravitational fields' occur, depending on the coordinate choice; it is not the fields themselves that lay claim to reality. They are merely analytical auxiliaries in the description of realities; in principle, one can actually only learn something about the latter by eliminating the coordinates. The ghost of absolute space haunts your rod example. The argument works ad hominem but does not hit the mark at all as I interpret it. It is not a question of a violation of logic. That a rod must either be straight or bent but cannot be both corresponds exactly to the objection advanced by philosophers against the special theory of rel. that the same body (at the same 〈instant spacetime point) cannot be simultaneously at rest and in motion (Einstein to Mie, Feb. 8, 1918; CPAE, Vol. 8, Doc. 460; my emphasis).

Mie's mistake was after all a sort of a dynamical version of the same conceptual confusions that we have encountered in the previous pages. In his lectures, Mie imagined (Fig. 5) that someone constructed a rectangular coordinate net (net I) using light rays (Mie 1917a, 599). One can substitute this net with another curvilinear coordinate system (net II), which would correspond to the presence of a gravitational field. The light
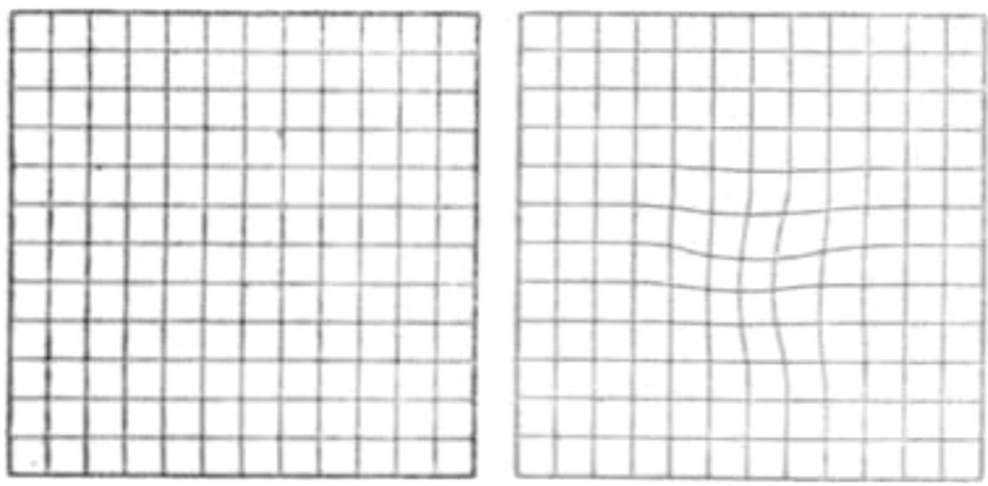

Fig. 5 Two equivalent coordinate systems, net I and net II (Mie 1917a) 
rays that appear optically straight in the first coordinate system would appear curved in the second coordinate system. According to the general relativity principle, net I is just as good as net II. However, this cannot be true. If someone introduces a gravitational field that changes periodically, light rays will appear as if they were moving like a vibrating string. ${ }^{43}$ According to Mie, no one would take this result seriously. If all coordinate systems are on the same footing mathematically, physically, the net I will be a more reasonable coordinate system that represents the 'real' path of light rays. Given this premise, it is not surprising that Einstein relied once again on the point-coincidence argument to convince his interlocutor that this was not the case. Light rays will follow the same paths and intersect at the same angles in all coordinate nets, I and II, or whatever oscillation between them one can imagine. Thus, they will produce the same interference patterns on a photographic plate, that is, the same coincidences.

Mie had just discussed this issue with the young Königsberg mathematician, Kretschmann (Mie to Einstein, Feb. 17, 1918; CPAE, Vol. 8, Doc. 465). Thus, in his reply, Mie alerted Einstein that the latter had shown that the point-coincidence argument was actually meaningless. In any theory, observations are reducible to point-coincidences, and thus any theory can be presented in an arbitrary coordinate system without changing its physical content (Mie to Einstein, Feb. 17, 1918; CPAE, Vol. 8, Doc. 465). In a letter written in May, as Kretschmann's paper (1917) was already published, Mie tried to defend his point of view by pointing out that it was not dissimilar to the one Einstein had introduced in his 1916 gravitational wave paper. As we have seen, Einstein (1916d) had, in fact, insisted that there was 'a deep physical justification' for imposing the condition $\sqrt{-g}=1$. As Mie pointed out, it was roughly the same attitude that he himself had taken in his Göttingen lectures. In particular, Einstein's 'deep physical justification' was “also almost exactly the same one I have explained with the slithering rod" (Mie to Einstein, Feb. 17, 1918; CPAE, Vol. 8, Doc. 465). Searching for gravitational wave solutions, Einstein simply came upon a coordinate system in which such a vibrating rod would occur, and he rejected this " "undulatory oscillating coordinate system' as nonphysical" (Mie to Einstein, Feb. 17, 1918; CPAE, Vol. 8, Doc. 532).

A mathematician, for sure, can consider all coordinate systems equivalent. After all, they are arbitrary numbering systems. However, a physicist, extracting quantities measurable with rods and clocks, light rays, and so forth from those numbers, is bound to prefer more reasonable coordinate systems over others (Mie 1920; 1921). In particular, one can never ascribe the same legitimacy to a coordinate system in which what Einstein called 'apparent' and Mie 'fictitious' gravitational phenomena exist-

\footnotetext{
${ }^{43}$ In our preferred analogy, Mie's thought experiment corresponds to the case of a sort of a dynamical map projection of Earth, which changes cyclically and smoothly from, say, cylindrical to stereographic, and then back again to cylindrical, on the same flat projection plane. Three cities that lie on a vertical line do not do the same in a stereographic projection. Thus, in our dynamical map, the line connecting them would appear to move in a snake-like fashion on the projection plane. Again, the illusion emerges if one takes the coordinates $x, y$ on the projection place as real distances. All these maps represent the same intrinsic geometrical structure of the globe. Most physicists seemed to think in terms of the maps, not in terms of the globe.
} 
for example, gravitational waves that do not transport energy. All coordinate systems are equivalent "in principle""; by assuming the mathematician's standpoint, one can define everything "on sovereign authority" (Mie to Einstein, Feb. 17, 1918; CPAE, Vol. 8, Doc. 465). However, from a physical point of view, the coordinate system in which the rod is not flapping is clearly better than the one in which it is not: "one can see clearly from this exactly why, as you say, 'there is profound physical justification for a coordinate choice' according to the condition $\sqrt{-g}=1 \mathrm{~s}$ " (Mie to Einstein, Feb. 17, 1918; CPAE, Vol. 8, Doc. 465). However, Einstein seemed to have now abandoned even the last trace of the materiality of the coordinate system. By changing the coordinates, one changes the form of the solution, but not its physical meaning. The distinction between a coordinate system being 'good' only for calculations, and a coordinate system being 'good' from a physical point of view could not be upheld. A plane wave, obtained by imposing the coordinate condition (5), becomes more complicated with a different choice of coordinates. Nevertheless, all gravitational waves that differ only by a coordinate transformation are physically the same. They produce the same elliptical oscillation in the distribution of suspended mirrored test masses, thus the same interference patterns in a gravitational wave detector. Ultimately, they produce the same 'coincidences'.

\section{Conclusion: the immateriality of the coordinate system}

On May 24, the Annalen published a brief programmatic paper in which Einstein, among other things, replied to Kretschmann's 'triviality-objection' against the point-coincidence argument. Einstein, so to speak, doubled down and elevated the point-coincidence argument to a fundamental physical principle in physics, the relativity principle (Gutfreund and Renn 2015): "Nature's laws are merely statements about temporal-spatial coincidences" (Einstein 1918b, 241). The requirement that only point-coincidences are observable, as the physical expression of the relativity principle, seems to have become independent of general covariance as a formal requirement (Lehner 2005). It is indeed possible that Kretschmann's objection did not catch Einstein by surprise. As far as I can see, it was never noticed that Einstein was aware early on that any theory can be trivially presented in a way in which coordinates are meaningless parameters (as discussed in Sections 2.1 and 2.2) without an operational interpretation. ${ }^{44}$ The point of issue was a different one.

In previous theories, there was a way to eliminate the parameters; thus, it was simpler to formulate the laws of nature with respect to a class of privileged coordinate systems in which coordinate differences are physically meaningful numbers resulting from rods-and-clocks measurements. If one "demands this, then one confers to the magnitudes $x, y, z, t$ physical meaning"; this is "convenient (although not logically necessary)" (Einstein to Adler, Aug. 4, 1918; CPAE, Vol. 8, Doc. 594; my emphasis). However, such 'deparameterization' is never possible in general relativity. Because of the equivalence principle, one has lost the ability to distinguish good,

\footnotetext{
${ }^{44}$ This corresponds to what Bergmann has called 'weak general covariance' (Bergmann 1957, 11).
} 
nonaccelerated rods and clocks that reliably measure coordinates from the bad accelerating ones whose measurement cannot be trusted. Einstein, with a creative leap of logic, concluded that there was no reason to prefer one coordinate system over another. The impossibility of formulating the theory with reference to a privileged class of coordinate systems gave to the "the relativity postulate [...] a heuristic meaning in spite of the apparent lack of real content" (Einstein 1918b, deleted passage). While general-relativistic theories, as any other fundamental theory, cannot dispense with coordinates, they are singled out by the fact that they can only use coordinates as nonmeasurable variables (Einstein 1918a, 699). ${ }^{45}$ A solution of Maxwell's equations $\varphi_{\mu \nu}\left(x_{v}\right)$ is physically meaningful only once one specifies how the parameters $x_{v}$ are measurable; on the contrary, the physical meaning of a solution of Einstein's equations $g_{\mu \nu}\left(x_{v}\right)$ is what remains once the dependence on the variables $x_{v}$ is eliminated.

The fact that Einstein's answer to Kretschmann appears to be nearly incomprehensible to the modern reader is possibly the consequence of our unfamiliarity with Einstein's algebraic, nongeometrical way of thinking. Einstein reasoned in terms of (kinematic and dynamic) variables that enter into the equations of physics and of the procedures through which these variables can be measured. In general-relativistic theories "the connection between quantities in equations and measurable quantities is far more indirect than in the customary theories of old" (Einstein 1918a, 700). In general relativity, it is impossible to devise an experiment that will measure whether the Ricci scalar $R=0$ at a point with the coordinates $x_{v}$, because the values of the coordinates by themselves are not measurable. However, one can ask whether or not $R=0$ at the point where two geodesics intersect. The point-coincidence argument was the operationalist answer to what was perceived as an operationalist conundrum, the impossibility to interpret the coordinates as readings on rods and clocks. The components $g_{\mu \nu}$ of the gravitational field at a certain location $x, y, z, t$ are not measurable; they represent "nothing physically real"; only "the gravitational field together with other data" is real (Einstein 1918a, 701).

In this sense, the point-coincidence argument, far from being a mere trick to escape from the hole argument, can be considered as Einstein's mature stance toward what is actually observable in physics. "Physical experiences [are] always assessments of point-coincidences (spacetime coincidences)" (Einstein 1919, [p. 3]). As far as I can see, this was Einstein's last formulation of the point-coincidence argument. A nonrelativistic theory, one might say, contains not only statements about coincidences, but also statements about the coordinate systems that serve for their description. On the contrary, a general-relativistic theory entails only relationships between coincidences, the statements of which are independent of the choice of coordinates. Although confusions about the role of coordinates in general relativity seem to have been quite widespread among relativists for several years (Darrigol 2015), the battle could be considered won (Einstein 1920). Einstein, moving beyond general relativity toward the unified field theory program, seems to have lost interest in the 'operationalist' rhetoric of the observability of coincidences. The point-coincidence

\footnotetext{
${ }^{45}$ This is 'strong general covariance' in Bergmann's parlance (Bergmann 1957, 11).
} 
argument is not mentioned in his Princeton lectures (Einstein 1922), Einstein's only presentation of the theory in textbook form.

Operationalism was for Einstein a 'throwaway philosophy' designed to be discarded once it played its iconoclastic role. As I plan to show elsewhere, it was Einstein's former assistant, Bergmann, who, in the 1950s, brought the language of 'coincidences' to the fore again. Einstein's 'operationalist' notion of 'coincidence' as the encounters of light rays and material particles was transformed into the 'realistic' notion of 'coincidence' as joint reading of field values (Bergmann 1961, 1962; Bergmann and Komar 1962). The value of the Ricci scalar $R$ at a worldpoint with coordinates $x_{v}$ is not an observable quantity in general relativity. However, if there is matter, one can measure the value of the scalar $R$ where four scalar functions of some matter field take such-and-such values. Where no matter is present, in principle one can construct four scalars from the independent degrees of freedom of the pure gravitational field (Komar 1958). In this sense, "[a] physically meaningful statement about events is necessarily a statement about coincidences," the joint measurements of the values of at least five scalars (Bergmann and Komar 1962, 315; see also Bergmann 1961, 1962). The worldpoints and their labels $x_{v}$ have no physical meaning behind these readings. "Perhaps this recognition will turn out to be of value in the years to come ..." (Bergmann 1980, 176).

Acknowledgements I am greatly indebted to Anne J. Kox for sharing the drafts of his edition of the Lorentz-Ehrenfest correspondence. I would like to thank Jim Weatherall, Sam Fletcher, Dennis Lehmkuhl for illuminating discussions of early versions of the paper. Additional special thanks go to Sam Fletcher for his careful reading of the final manuscript and his many insightful comments and suggestions.

Funding Open access funding provided by Università degli Studi di Torino within the CRUI-CARE Agreement.

Abbreviations CPAE, Albert Einstein (1987-). The collected papers of Albert Einstein. Ed. by John Stachel et al. 15 vols. Princeton: Princeton University Press, 1987-; EA, The Albert Einstein Archives at the Hebrew University of Jerusalem; SCHAL, Hendrik Antoon Lorentz (2008-). The scientific correspondence of H.A. Lorenzt. Ed. by A. J. Kox. New York: Springer, 2008-. I thank A.J. Kox for giving me access to the unpublished vol. 2 .

Open Access This article is licensed under a Creative Commons Attribution 4.0 International License, which permits use, sharing, adaptation, distribution and reproduction in any medium or format, as long as you give appropriate credit to the original author(s) and the source, provide a link to the Creative Commons licence, and indicate if changes were made. The images or other third party material in this article are included in the article's Creative Commons licence, unless indicated otherwise in a credit line to the material. If material is not included in the article's Creative Commons licence and your intended use is not permitted by statutory regulation or exceeds the permitted use, you will need to obtain permission directly from the copyright holder. To view a copy of this licence, visit http://creativecommonshorg/licenses/by/4. $0 /$.

\section{References}

Abraham, M. (1912a). Nochmals Relativität und Gravitation. Bemerkungen zu A. Einsteins Erwiderung. Annalen der Physik. 4th ser., 39, 444-448. 
Abraham, M. (1912b). Zur Theorie der Gravitation. Physikalische Zeitschrift, 13, 1-4.

Abraham, M. (1914). Neuere Gravitationstheorien. Jahrbuch der Radioaktivität und Elektronik, 11(3), $470-520$

Anderson, J.L., \& Finkelstein, D. (1971). Cosmological constant and fundamental length. American Journal of Physics, 39, 901-904.

Bachelard, G. (1938). La Formation de l'esprit scientifique. Contribution à une psychanalyse de la connaissance objective. Paris: Vrin, 1938.

Bergmann, P.G. (1957). Topics in the theory of general relativity. Lectures in theoretical physics. Brandeis Summer Institute. New York: W. A. Benjamin, pp. 1-44.

Bergmann, P.G. (1961). Observables in general relativity. Reviews of modern physics, 33, 510-514.

Bergmann, P.G. (1962). The general theory of relativity. In Flügge, S. (Ed.) Handbuch der Physik / Encyclopedia of physics, vol. 2/4: Principles of electrodynamics and relativity / Prinzipien der elektrodynamik und relativitästheorie. 55 vols. (pp. 203-272). Berlin: Springer.

Bergmann, P.G. (1980). The fading world point. In Bergmann, P.G., \& De Sabbata, V. (Eds.) Cosmology and gravitation. Spin, torsion, rotation, and supergravity (pp. 173-176). New York: Plenum Press.

Bergmann, P.G., \& Komar, A.B. (1962). Observables and commutation relations. In Lichnerowicz, A., \& Tonnelat, M.-A. (Eds.) Les théories relativistes de la gravitation. Actes du colloque tenu à Royaumont, 21-27 Juin 1959 (pp. 309-325). Paris: Éd. du centre national de la recherche scientifique.

Bergmann, P.G., \& Smith, G.J. (1982). Measurability analysis of the linearized gravitational field. General Relativity and Gravitation, 14, 1131-1166.

Born, M. (1909). Über die Dynamik des Elektrons in der Kinematik des Relativitätsprinzips. Physikalische Zeitschrift, 10, 814-817.

Cassirer, E. (1921). Zur Einsteinschen Relativitätstheorie. Erkenntnistheoretische Betrachtungen. Berlin: B. Cassirer, 1921.

Darrigol, O. (2015). Mesh and measure in early general relativity. Studies in history and philosophy of science. Part B: Studies in history and philosophy of modern physics, 52, 163-187.

De Sitter, W. (1916a). On Einstein's theory of gravitation and its astronomical consequences. First paper. Monthly notices of the Royal Astronomical Society, 76, 699-728.

De Sitter, W. (1916b). Space, time, and gravitation. The observatory, 39, 412-419.

De Sitter, W. (1917). Planetary motion and the motion of the moon according to Einstein's theory. Verhandelingen der Koninklijke Akademie van Wetenschappen te Amsterdam, 19, 367-381.

Droste, J. (1914). On the of field of a single centre in Einstein's theory of gravitation. Verhandelingen der Koninklijke Akademie van Wetenschappen te Amsterdam, 17, 998-1011.

Droste, J. (1915). On the field of two spherical fixed centres in Einstein's theory of gravitation. Verhandelingen der Koninklijke Akademie van Wetenschappen te Amsterdam, 18, 760-769.

Droste, J. (1917). The field of a single centre in Einstein's theory of gravitation, and the motion of a particle in that field. Verhandelingen der koninklijke Akademie van Wetenschappen te Amsterdam, 19, $197-215$

Earman, J. (1989). World enough and space-time. Absolute vs. relational theories of space and time. Cambridge: MIT Press.

Earman, J., \& Glymour, C. (1980a). Relativity and eclipses. Historical Studies in the Physical Sciences, $11,49-85$.

Earman, J., \& Glymour, C. (1980b). The gravitational red shift as a test of general relativity. History and analysis. Studies in history and philosophy of science, 11, 175-214.

Earman, J., \& Norton, J.D. (1987). What price substantivalism? The Hole story. The British journal for the philosophy of science, 38, 515-525.

Ehrenfest, P. (1909). Gleichförmige Rotation starrer Körper und Relativitätstheorie. Physikalische Zeitschrift, 10,918.

Einstein, A. (1905). Über einen die Erzeugung und Verwandlung des Lichtes betreffenden heuristischen Gesichtspunkt. Annalen der Physik. 4th ser, 17, 132-148. Repr. in CPAE, Vol. 2, Doc. 14.

Einstein, A. (1907a). Bemerkungen zu der Notiz von Hrn. Paul Ehrenfest: Die Translation deformierbarer Elektronen und der Flächensatz. Annalen der Physik. 4th ser, 22, 206-208. Repr. in CPAE, Vol. 2, Doc. 44.

Einstein, A. (1907b). Über die vom Relativitätsprinzip geforderte Trägheit der Energie. Annalen der Physik. 4th ser., 23, 371-384. Repr. in CPAE, Vol. 2, Doc. 45.

Einstein, A. (1908). Relativitätsprinzip und die aus demselben gezogenen Folgerungen. Jahrbuch der Radioaktivität und Elektronik, 4, 411-462. Repr. in CPAE, Vol. 2, Doc. 47. 
Einstein, A. (1909a). Über die Entwicklung unserer Anschauungen. Über das Wesen und die Konstitution der Strahlung. Vorgetragen in der Sitzung der physikalischen Abteilung der 81. Versammlung Deutscher Naturforscher und Ärzte zu Salzburg am 21. September 1909 (Vol. 7 pp. 482-500). Repr. in CPAE, Vol. 2, Doc. 60. Also pub Physikalische zeitschrift, 10, 1909, 817-825.

Einstein, A. (1909b). Über die Entwicklung unserer Anschauungen über das Wesen und die Konstitution der Strahlung. Vorgetragen in der Sitzung der physikalischen Abteilung der 81. Versammlung Deutscher Naturforscher und Ärzte zu Salzburg am 21, September 1909. Physikalische Zeitschrift, 10, 817-825. Repr. in CPAE Vol. 1 Doc. 60.

Einstein, A. (1910a). Le principe de relativité et ses conséquences dans la physique moderne. Trans. by Edouard Guillaume. Archives des sciences physiques et naturelles, 29, 5-28, 125-144. Repr. in CPAE, Vol. 3, Doc. 2.

Einstein, A. (1910c). Theorie der Opaleszenz von homogenen Flüssigkeiten und Flüssigkeitsgemischen in der nähe des kritischen Zustandes. Annalen der Physik. 4th ser., 14, 1276-1298.

Einstein, A. (1911a). [Statement on the light quantum hypothesis]. Sitzungsberichte der Naturforschenden Gesellschaft in Zürich, XVI. Pub. in CPAE, Vol. 3, Doc. 20.

Einstein, A. (1911b). Die Relativitäts-Theorie. Naturforschende Gesellschaft in Zürich. Vierteljahresschrift, 56, 1-14. Repr. in CPAE, Vol. 3, Doc. 17.

Einstein, A. (1911c). Diskussion von Die Relativitäts-Theorie [Einstein, 1911b]. Naturforschende Gesellschaft in Zürich, Vierteljahresschrift, 56, II-IX. Repr. in CPAE, Vol. 3, Doc. 18.

Einstein, A. (1911d). Einfluss der Schwerkraft auf die Ausbreitung des Lichtes. Annalen der Physik. 4th ser., 35, 898-908. Repr. in CPAE, Vol. 3, Doc. 23.

Einstein, A. (1911e). Zum Ehrenfestschen Paradoxon. Bemerkung zu V. Varićak Aufsatz. Physikalische Zeitschrift, 12, 509-510. Repr. in CPAE, Vol. 3, Doc. 22.

Einstein, A. (1912). Einstein's manuscript on the special theory of relativity. Pub. in CPAE, Vol. 4, Doc. 1.

Einstein, A. (1912a). Lichtgeschwindigkeit und Statik des Gravitationsfeldes. Annalen der Physik. 4th ser, 38, 355-369. Repr. in CPAE, Vol. 4, Doc. 3.

Einstein, A. (1912b). Relativität und Gravitation. Erwiderung auf eine Bemerkung von M. Abraham. Annalen der Physik. 4th ser, 38, 1059-1064. Repr. in CPAE, Vol. 4, Doc. 8.

Einstein, A. (1912c). Theorie des statischen Gravitationsfeldes. Annalen der Physik. 4th ser, 38, 443-458. Repr. in CPAE, Vol. 4, Doc. 4.

Einstein, A. (1913a). Gravitationstheorie. Schweizerische Naturforschende Gesellschaft, Verhandlungen, 96, 137-138. Repr. in CPAE, Vol. 15, Doc. 4.

Einstein, A. (1913b). Zum gegenwärtigen Stande des Gravitationsproblems. Physikalische Zeitschrift, 14, 1249-1266. Repr. in CPAE, Vol. 4, Doc. 17.

Einstein, A. (1914a). Die formale Grundlage der allgemeinen Relativitätstheorie. Sitzungsberichte der Preußischen Akademie der Wissenschaften, Halbband, 2, 1030-1085. Repr. in CPAE, Vol. 6, Doc. 9.

Einstein, A. (1914b). Prinzipielles zur verallgemeinerten Relativitätstheorie. Physikalische Zeitschrift, 15, 176-180. Repr. in CPAE, Vol. 4, Doc. 25.

Einstein, A. (1915a). Erklärung der Perihelbewegung des Merkur aus der allgemeinen Relativitätstheorie. Sitzungsberichte der Preußischen Akademie der Wissenschaften, Halbband 2, 831-839, Repr. in CPAE, Vol. 6, Doc. 24.

Einstein, A. (1915b). Feldgleichungen der Gravitation. Sitzungsberichte der Preußischen Akademie der Wissenschaften, Halbband 2, 844-847, Repr. in CPAE, Vol. 5, Doc. 25.

Einstein, A. (1915c). Zur allgemeinen Relativitätstheorie. Sitzungsberichte der Preußischen Akademie der Wissenschaften, Halbband 2, 778-786, Repr. in CPAE, Vol. 6, Doc. 21.

Einstein, A. (1915d). Zur allgemeinen Relativitätstheorie (Nachtrag). Sitzungsberichte der Preußischen Akademie der Wissenschaften, Halbband 2, 799-801, Repr. in CPAE, Vol. 6, Doc. 22.

Einstein, A. (1916a). Die Grundlage der allgemeinen Relativitätstheorie. Annalen der Physik. 4th ser, 49, 769-822. Also issued as a separatum, Leipzig: Barth. Repr. in CPAE, Vol. 6, Doc. 30.

Einstein, A. (1916b). Eine neue formale Deutung der Maxwellschen Feldgleichungen der Elektrodynamik. Sitzungsberichte der Preuß, ischen Akademie der Wissenschaften, 184-188.

Einstein, A. (1916c). Hamiltonsches Prinzip und allgemeine Relativitätstheorie. Sitzungsberichte der Preußischen Akademie der Wissenschaften, 1111-1116, Repr. in CPAE, Vol. 6, Doc. 41.

Einstein, A. (1916d). Näherungsweise Integration der Feldgleichungen der Gravitation. Sitzungsberichte der preußischen Akademie der Wissenschaften, Halbband 1, pp 688-696.

Einstein, A. (1916e). Strahlungs- Emission und -Absorption nach der Quantentheorie. Verhandlungen der Deutschen Physikalischen Gesellschaft, 18, 318-323. Repr. in CPAE, Vol. 6, Doc. 34. 
Einstein, A. (1916f). Über die spezielle und die allgemeine Relativitätstheorie (gemeinverständlich). Line Braunschweig: Vieweg, Repr. in CPAE, Vol. 5, Doc. 42.

Einstein, A. (1916g). Über Fr. Kottlers Abhandlung: Einsteins Äquivalenzhypothese und die Gravitation. Annalen der Physik. 4th ser., 51, 239. Repr. in CPAE, Vol. 6, Doc. 40.

Einstein, A. (1916h). Zur Quantentheorie der Strahlung. Mitteilungen der physikalischen Gesellschaft Zürich, 18, 47-62. Repr. in CPAE, Vol. 6, Doc. 38.

Einstein, A. (1917a). Kosmologische Betrachtungen zur allgemeinen Relativitätstheorie. Sitzungsberichte der Preußischen Akademie der Wissenschaften, Halbband 1, 142-152, Repr. in CPAE, Vol. 6, Doc. 43.

Einstein, A. (1918a). Dialog über Einwände gegen die Relativitätstheorie. Die Naturwissenschaften, 6, 697-702. Repr. in CPAE, Vol. 7, Doc. 13.

Einstein, A. (1918b). Prinzipielles zur allgemeinen Relativitätstheorie. Annalen der Physik. 4th ser., 55, 241-244. Repr. in CPAE, Vol. 7, Doc. 4.

Einstein, A. (1918c). Über Gravitationswellen. Sitzungsberichte der Preußischen Akademie der Wissenschaften, 154-167.

Einstein, A. (1919). Lecture notes for course on general relativity at the university of Berlin. Summer semester, 1919, Pub. in CPAE, Vol. 7, Doc. 19.

Einstein, A. (1920). Antwort auf vorstehende Betrachtung [Reichenbächer, 1920]. Die Naturwissenschaften, 8, 1010-1011. Repr. in CPAE, Vol. 7, Doc. 49.

Einstein, A. (1922). Vier Vorlesungen über Relativitätstheorie, gehalten im Mai 1921, an der Universität Princeton. Braunschweig: Vieweg, 1922, Repr. in CPAE, Vol. 7, Doc. 71.

Einstein, A. (1923). Vorwort des Autors zur Tschechischen Ausgabe. In Theorie relativity speciálni $i$ obecna. Lehce srozumitelný výklad. Praha: Borový, 1923. written in 1922 for the 1923 Czech edition of Einstein, 1917b; cited in CPAE, Vol. 6, Doc. 53, note 4.

Einstein, A. (1933). Einiges über die Entstehung der allgemeinen Relativitätstheorie, EA, 5-3.

Einstein, A., \& Fokker, A.D. (1914). Die Nordströmsche Gravitationstheorie vom Standpunkt des absoluten Differentialkalküls. Annalen der Physik. 4th ser, 44, 321-328. Repr. in CPAE, Vol. 4, Doc. 28.

Einstein, A., \& Grossmann, M. (1913). Entwurf einer verallgemeinerten Relativitätstheorie und eine Theorie der Gravitation. I: Physikalischer Teil von A. Einstein. II: Mathematischer Teil von M. Grossmann. Leipzig: Teubner, 1913. Repr. in CPAE, Vol. 4, Doc. 13.

Einstein, A., \& Grossmann, M. (1914a). Entwurf einer verallgemeinerten Relativitätstheorie und eine Theorie der Gravitation. I: Physikalischer Teil von A. Einstein. II: Mathematischer Teil von M. Grossmann. Zeitschrift für Mathematik und Physik 62, 225-44, 245-261. With additional Bemerkungen; repr. in CPAE, Vol. 4, Doc. 26.

Einstein, A., \& Grossmann, M. (1914b). Kovarianzeigenschaften der Feldgleichungen der auf die verallgemeinerte Relativitätstheorie gegründeten Gravitationstheorie. Zeitschrift für Mathematik und Physik, 63, 215-225. Repr. in CPAE, Vol. 6, Doc. 2.

Einstein, A., \& Infeld, L. (1938). The evolution of physics. The growth of ideas from early concepts to relativity and quanta, New York: Simon/Schuster, 1938.

Engler, F.O., \& Renn, J. (2013). Hume, Einstein und Schlick über die Objektivität der Wissenschaft. In Engler, F. O. and Mathias, I. (Eds.) Moritz Schlick - Die Rostocker Jahre und ihr Einfluss auf die Wiener Zeit. Die Rostocker Jahre und ihr Einfluss auf die Wiener Zeit, Leipzig: Leipziger Universitätsverlag 2013, 123-156.

Frank, P. (1908). Das Relativitätsprinzip der Mechanik und die Gleichungen für die elektromagnetischen vorgänge in bewegten Körpern. Annalen der Physik, 332, 897-902.

Friedman, M. (1983). Foundations of space-time theories. Relativistic physics and philosophy of science. Princeton: Princeton University Press.

Geiser, C.F. (1897). Infinitesimalgeometrie I. Vorlesung von Carl Friedrich Geiser. Vorlesungsnachschrift, ausgearbeitet von Marcel Grossmann. 1897-1898. ETH-Bibliothek Zürich, Hs 421:15.1.

Giovanelli, M. (2013). Erich Kretschmann as a proto-logical-empiricist. Adventures and misadventures of the point-coincidence argument. Studies in history and philosophy of science. Part B: Studies in history and philosophy of modern physics, 44, 115-134.

Giovanelli, M. (2014). But One Must Not Legalize the Mentioned Sin. Phenomenological vs. Dynamical Treatments of Rods and Clocks in Einstein's Thought. Studies in history and philosophy of science. Part B: Studies in history and philosophy of modern physics, 48, 20-44. 
Giovanelli, M. (2016). .... But i still can't get rid of a sense of artificiality: the Einstein-Reichenbach debate on the geometrization of the electromagnetic field. Studies in history and philosophy of science. Part B: Studies in history and philosophy of modern physics, 54, 35-51.

Giovanelli, M. (2018). Physics is a kind of metaphysics. Émile Meyerson and Einstein's late rationalistic realism. European Journal for philosophy of science, 8, 783-829.

Giovanelli, M. (2020). Like thermodynamics before Boltzmann. On Einstein's distinction between constructive and principle theories. Studies in history and philosophy of science. Part B: Studies in history and philosophy of modern physics, 71, 118-157.

Gutfreund, H., \& Renn, J. (2015). The road to relativity. The history and meaning of Einstein's The foundation of general relativity. Featuring the original manuscript of Einstein's masterpiece. Princeton: Princeton University Press.

Herglotz, G. (1911). Über die Mechanik des deformierbaren Körpers vom Standpunkte der Relativitätstheorie. Annalen der Physik. 4th ser., 36, 493-533.

Hilbert, D. (1915). Grundlagen der Physik. Erste Mitteilung, vorgelegt in der Sitzung vom 20. November 1915. Nachrichten von der Königlichen Gesellschaft der Wissenschaften zu Göttingen, Mathematischphysikalische Klasse, 395-407. Repr. in Hilbert, 2009, ch, I, 28-46.

Hilbert, D. (2009). David Hilbert's Lectures on the foundations of physics 1915-1927, ed. by Sauer, T. and Majer, U. Berlin/Heidelberg: Springer.

Hoffmann, B. (1982). Some Einstein anomalies. In Holton, G., \& Elkana, Y. (Eds.) Albert Einstein, historical and cultural perspectives. The centennial symposium in Jerusalem (pp. 91-105). Princeton: Dove.

Howard, D. (1991). Einstein and Eindeutigkeit. A neglected theme in the philosophical background to general relativity. In Eisenstaedt, J., \& Kox, A.J. (Eds.) Historical studies in general relativity (pp. 154-243). Birkhäuser: Boston.

Howard, D. et al. (1999). Point coincidences and pointer coincidences. Einstein on the invariant content of space-time theories. In Goenner, H.F.M. (Ed.) The expanding worlds of general relativity (pp. 463500). Birkäuser: Boston.

Howard, D., \& Norton, J.D. (1993). Out Of the labyrinth. Einstein, Hertz, and the Göttingen answer to the hole argument. In Howard, D., \& Stachel, J. (Eds.) Einstein and the history of general relativity. Proceedings of the 1986 Osgood Hill Conference, North Andover, Massachusetts, May 8 to 11, 1986 (pp. 30-62). Birkhäuser: Boston.

Illy, J. (1979). Albert Einstein in Prague. Isis, 70, 76-84.

Illy, J. (1988). The correspondence of Albert Einstein and Gustav Mie, 1917-1918. In Eisenstaedt, J., \& Kox, A.J. (Eds.) Studies in the history of general relativity (pp. 244-259). Birkhäuser: Boston.

Janssen, M. (1998). Einstein-De Sitter debate. https://www.ilorentz.org/research/vanbaal/DECEASED/ ART/E-dS.pdf.

Janssen, M. (2003). A glimpse behind the curtain of the wizard/Un coup d'oeil derriére le rideau du magicien. In Lhéritier, G. (Ed.) Paris: Scriptura/ Aristophile.

Janssen, M. (2005). Of pots and holes. Einstein's bumpy road to general relativity. Annalen der Physik 14 Supplement, 58-85.

Janssen, M. (2007). What did Einstein know and when did he know it? A Besso memo dated August 1913. In Renn, J. (Ed.) The genesis of general relativity, (Vol. 4 pp. 787-837). Dordrecht: Springer.

Janssen, M., \& Renn, J. (2007). Untying the knot. How Einstein found his way back to field equations discarded in the Zurich notebook. In Renn, J. (Ed.) The Genesis of general relativity, (Vol. 4 pp. 839925). Dordrecht: Springer.

Janssen, M., \& Renn, J. (2015a). Arch and scaffold. How einstein found his field equations. Physics today, 68, 30-36.

Janssen, M., \& Renn, J. (2015b). Einstein was no lone genius. Nature, 527, 298-300.

Kaluza, T. (1910). Zur Relativitätstheorie. Physikalische Zeitschrift, 11, 977-978.

Kennefick, D. (2007). Traveling at the speed of thought. Einstein and the quest for gravitational waves. Princeton: Princeton University Press.

Komar, A.B. (1958). Construction of a complete set of independent observables in the general theory of relativity. Physical Review Letters, 111, 1182-1187.

Kox, A.J. (1985). H. A. Lorentz and general relativity. Manuscript. ICHS, Berkeley.

Kox, A.J. (1992). General relativity in the Netherlands, 1915-1920. In Eisenstaedt, J., \& Kox, A.J. (Eds.) Studies in the history of general relativity (pp. 39-56). Birkhäuser: Basel/Boston. 
Kretschmann, E. (1915). Über die prinzipielle Bestimmbarkeit der berechtigten Bezugssysteme beliebiger Relativitätstheorien. Annalen der Physik. 4th ser., 23, I: 907-942, II: 943-982.

Kretschmann, E. (1917). Über den physikalischen Sinn der Relativitätspostulate. A. Einsteins neue und seine ursprüngliche Relativitätstheorie. Annalen der Physik. 4th ser., 53, 575-614.

Lehmkuhl, D. (2019). The equivalence principle(s). In Knox, E., \& Wilson, A. (Eds.) The Routledge companion to philosophy of physics. London: Routledge. forthcoming.

Lehner, C. (2005). Einstein and the principle of general relativity, 1916-1921. In Kox, A.J., \& Eisenstaedt, J. (Eds.) The universe of general relativity, (Vol. 2005 pp. 103-108). Birkhäuser: Boston.

Levi-Civita, T., \& Ricci-Curbastro, G. (1900). Méthodes de calcul diffürentiel absolu et leurs applications. Mathematische Annalen, 54, 125-201.

Lorentz, H.A. (1915). On Hamilton's principle in Einstein's theory of gravitation. Verhandelingen der Koninklijke Akademie van Wetenschappen te Amsterdam, 19, 751-767.

Lorentz, H.A. (1917a). De gravitatietheorie van Einstein en de grondbegrippen der natuurkunde. De Ingenieur, 32, 649-655.

Lorentz, H.A. (1917b). On Einstein's theory of gravitation, I. Verhandelingen der Koninklijke Akademie van Wetenschappen te Amsterdam, 19, 1341-1354.

Lorentz, H.A. (1917c). On Einstein's theory of gravitation, II. Verhandelingen der Koninklijke Akademie van Wetenschappen te Amsterdam, 19, 1354-1369.

Lorentz, H.A. (1918). On Einstein's theory of gravitation, III. Verhandelingen der Koninklijke Akademie van Wetenschappen te Amsterdam, 20, 2-19.

Maltese, G., \& Orlando, L. (1995). The definition of rigidity in the special theory of relativity and the genesis of the general theory of relativity. Studies in history and philosophy of science. Part B: Studies in history and philosophy of modern physics, 26, 263-306.

Mie, G. (1917a). Die Einsteinsche Gravitationstheorie und das Problem der Materie I. Physikalische Zeitschrift, 18, 551-556.

Mie, G. (1917b). Die Einsteinsche Gravitationstheorie und das Problem der Materie. III. Physikalische Zeitschrift, 18, 596-602.

Mie, G. (1917c). Die Einsteinsche Gravitationstheorie und das Problem der Materie. II. Physikalische Zeitschrift, 18, 574-580.

Mie, G. (1920). Die Einführung eines vernunftgemäßen Koordinatensystems in die Einsteinsche Gravitationstheorie und das Gravitationsfeld einer schweren Kugel. Annalen der Physik. 4th ser., 62, 46-74.

Mie, G. (1921). Die Einsteinsche Gravitationstheorie. Leipzig: Hirzel.

Minkowski, H. (1909). Raum und Zeit. Jahresberichte der Deutschen Mathematiker-Vereinigung, 18, 7588. Repr. in MGA, vol. 2, 431-446. Also pub. in: Physikalische Zeitschrift 10, 1909, 104-111.

Nordström, G. (1912). Relativitätsprinzip und Gravitation. Physikalische Zeitschrift, 13, 1126-1129.

Nordström, G. (1913a). Träge und schwere Masse in der Relativitätsmechanik. Annalen der Physik, 40, 856-878.

Nordström, G. (1913b). Zur Theorie der Gravitation vom Standpunkt des Relativitätsprinzips. Annalen der Physik. 4th ser., 42, 533-554.

Nordström, G. (1918a). On the energy of the gravitation field in Einstein's theory. Verhandelingen der Koninklijke Akademie van Wetenschappen te Amsterdam, 20, 1238-1245.

Nordström, G. (1918b). On the mass of a material system according to the gravitation theory of Einstein. Verhandelingen der Koninklijke Akademie van Wetenschappen te Amsterdam, 20, 1076-1091.

Norton, J.D. (1984). How Einstein found his field equations. 1912-1915. Historical studies in the physical sciences, 14, 253-316.

Norton, J.D. (1987). Einstein, the hole argument and the reality of space. In Forge, J. (Ed.) Measurement, realism, and objectivity. Essays on measurement in the social and physical sciences (pp. 153-188). Dordrecht, Boston/London: Kluwer.

Norton, J.D. (1992). Einstein, Nordström and the early demise of scalar, Lorentz-covariant theories of gravitation. Archive for history of exact sciences, 45, 17-94.

Norton, J.D. (1999). Geometries in collision. Einstein, Klein and Riemann. In Gray, J. (Ed.) The symbolic universe. Geometry and physics 189-1930 (pp. 128-144). Oxford New York: Oxford University Press.

Norton, J.D. (2002). Einstein's triumph over the spacetime coordinate system. A paper presented in honor of Roberto Torretti. Dialogos, 79, 253-262. 
Norton, J.D. (2005). Conjecture on Einstein, the independent reality of spacetime coordinate systems and the disaster of 1913. In Kox, A.J., \& Eisenstaedt, J. (Eds.) The universe of general relativity (pp. 67-102). Birkhäuser: Boston.

Ohanian, H.C. (1976). Gravitation and spacetime. New York: Norton.

Pais, A. (1982). Subtle is the Lord. The science and the life of Albert Einstein. New York: Oxford University Press.

Petzoldt, J. (1921). Die Stellung Der Relativitätstheorie in der geistigen Entwicklung der Menschheit. Dresden: Sibyllen.

Pooley, O. (2013). Substantivalist and relationalist approaches to spacetime. In Batterman, R. (Ed.) The Oxford handbook of philosophy of physics (p. 2013). Oxord: Oxford University Press.

Popper, K.R. (1929). Axiome, Definitionen und Postulate der Geometrie. 1929. The Karl Popper Archives, 4.6; repr. in Popper, 2006, 265-390.

Popper, K.R. (2006). Frühe Schriften. Ed. by Hansen, T.E. Tübingen: Mohr Siebeck.

Reich, K. (1994). Die Entwicklung des Tensorkalküls. Vom absoluten Differentialkalkül zur Relativitätstheorie. Berlin: Birkhäuser.

Reichenbach, H. (1924). Axiomatik der relativistischen Raum-Zeit-Lehre. Vieweg Braunschweig, 1924. Repr. in Reichenbach, 1977, 3, 3-171.

Reichenbach, H. (1928). Philosophie der Raum-Zeit-Lehre, Walter de Gruyter, Berlin/Leipzig. Repr. in Reichenbach, 1977, 2.

Reichenbach, H. (1977). Gesammelte Werke in 9 Bänden. Ed. by Kamlah, A., and Reichenbach, M. Braunschweig/Wiesbaden: Vieweg.

Reichenbächer, E. (1920). Inwiefern läßt sich die moderne Gravitationstheorie ohne die Relativität begründen? Die Naturwissenschaften, 8, 1008-1010.

Reissner, H. (1916). Über die Eigengravitation des elektrischen Feldes nach der Einsteinschen Theorie. Annalen der Physik, 355, 106-120.

Renn, J. (Ed.) (2007a). The genesis of general relativity, Vol. 4. Dordrecht: Springer.

Renn, J. (2007b). The summit almost scaled. Max Abraham as a pioneer of a relativistic theory of gravitation. In Renn, J. (Ed.) The genesis of general relativity, (Vol. 4 pp. 305-330). Dordrecht: Springer.

Renn, J., \& Sauer, T. (2007). Pathways out of classical physics. Einstein's double strategy in his search for the gravitational field equation. In Renn, J. (Ed.) The genesis of general relativity, (Vol. 4 pp. 113312). Dordrecht: Springer.

Rickles, D. (2007). Symmetry, structure, and spacetime. Amsterdam: Elsevier.

Rovelli, C. (2002). Partial observables. Physical Review D, 65, 124013.

Rowe, D.E. (2008). Max von Laue's role in the relativity revolution. The Mathematical Intelligencer, 30, 54-60.

Ryckman, T. (1992). (P)oint-(C)oincidence thinking. The ironical attachment of logical empiricism to general relativity. Studies in history and philosophy of science. Part B: Studies in History and Philosophy of Modern Physics, 23, 471-497.

Sauer, T. (2005). Albert Einstein's 1916 review article on general relativity. In Grattan-Guinness, I., \& Cooke, R. (Eds.) Landmark writings in western mathematics 1640-1940 (pp. 802-822). Amsterdam/Boston: Elsevier.

Sauer, T. (2015). Marcel Grossmann and his contribution to the general theory of relativity. In Rosquist, K., Jantzen, R.T., Ruffini, R. (Eds.) The thirteenth Marcel Grossmann meeting. Proceedings of the MG13 Meeting on General Relativity, Stockholm University, Sweden, July 1 to 7, 2012 (pp. 456-503). Singapore: World Scientific.

Schlick, M. (1917). Raum und Zeit in der gegenwärtigen Physik. Zur Einführung in das Verständnis der allgemeinen Relativitätstheorie. Die Naturwissenschaften, 5, 161-167 177-186. Repr. in Schlick, 2006, 2.

Schlick, M. (2006). Gesamtausgabe. Stadler, F., \& Wendel, H.J. (Eds.) Berlin: Springer.

Schrödinger, E. (1918). Die Energiekomponenten des Gravitationsfeldes. Physikalische Zeitschrift, 19, $161-166$.

Schwarzschild, K. (1916a). Über das Gravitationsfeld einer Kugel aus inkompressibler Flüssigkeit nach der Einsteinschen Theorie. Vorgelegt am 24. Februar 1916. Sitzungsberichte der preußischen Akademie der Wissenschaften. Physikalisch-mathematische Klasse, 424-434. 
Schwarzschild, K. (1916b). Über das Gravitationsfeld eines Massenpunktes nach der Einsteinschen Theorie. Vorgelegt am 13. Januar 1916. Sitzungsberichte der preußischen Akademie der Wissenschaften. Physikalisch-mathematische Klasse, 189-196.

Speziali, P. (1972). Albert Einstein-Michele Besso, Correspondance 1903-1955. Paris: Hermann.

Stachel, J. (1980). Einstein's search for general covariance, 1912-1915. 1980. The paper was first read at the Ninth International Conference on General Relativity and Gravitation, Jena in 1980; repr. in Stachel, 2002, 301-337.

Stachel, J. (1987). How Einstein discovered general relativity. A historical tale with some contemporary morals, general relativity and gravitation. In MacCallum, M.A.H. (Ed.) Proceedings of the 11th international conference on general relativity and gravitations (pp. 200-208). Cambridge: Cambridge University Press. Republished in Stachel, 2002, 293-300.

Stachel, J. (1989). The Rigidly rotating disk as the missing link in the history of general relativity. In Howard, D., \& Stachel, J. (Eds.) Einstein and the history of general relativity. Proceedings of the 1986 Osgood Hill Conference, North Andover, Massachusetts, May 8 to 11, 1986 (pp. 48-62). Birkhäuser: Boston.

Stachel, J. (1993). The meaning of general covariance. The hole story. In Earman, J. (Ed.) Philosophical problems of the internal and external worlds. Essays on the philosophy of Adolf Grünbaum (pp. 129160). Pittsburgh: University of Pittsburgh Press.

Stachel, J. (2002). Einstein from B to Z. Boston: Birkhäuser.

Stachel, J. (2014). The hole argument and some physical and philosophical implications. Living reviews in relativity 17. www.livingreviews.org/lrr-2014-1.

Study, E. (1914). Die realistische Weltansicht und die Lehre von Raume. Geometrie, Anschauung und Erfahrung. Braunschweig: F, Vieweg \& Sohn.

Torretti, R. (1983). Relativity and geometry. Oxford: Pergamon Press.

Valente, M.B. (2018). Perverted space-time geodesy in Einstein's views on geometry. Philosophia scientae, 22-2, 137-162.

Weatherall, J.O. (2018). Regarding the hole argument. The British journal for the philosophy of science, 69, 329-350.

Weinstein, G. (2015). General relativity conflict and rivalries. Einstein's polemics with physicists. Newcastle upon Tyne: Cambridge Scholars Publishing.

Westman, H., \& Sonego, S. (2008). Events and observables in generally invariant spacetime theories. Foundations of Physics, 38, 908-915.

Yavelov, B. (2002). Einstein's Zurich colloquium. In Balashov, Y., \& Vizgin, V.P. (Eds.) Einstein studies in Russia (pp. 261-295). Birkhüuser: Boston.

Publisher's note Springer Nature remains neutral with regard to jurisdictional claims in published maps and institutional affiliations. 